\title{
A molecular network of conserved factors keeps ribosomes dormant in the egg
}

Friederike Leesch ${ }^{1,2 \#}$, Laura Lorenzo-Orts ${ }^{1 \#}$, Carina Pribitzer ${ }^{1}$, Irina Grishkovskaya ${ }^{1}$, Manuel Matzinger $^{1}$, Elisabeth Roitinger ${ }^{1}$, Katarina Belačić ${ }^{1,2}$, Susanne Kandolf ${ }^{1}$, Tzi-Yang Lin ${ }^{1,2}$, Karl Mechtler ${ }^{1}$, Anton Meinhart ${ }^{1}$, David Haselbach ${ }^{1 *}$, Andrea Pauli ${ }^{1 *}$

(1) Research Institute of Molecular Pathology (IMP), Vienna BioCenter (VBC), Vienna, Austria

(2) Vienna BioCenter PhD Program, a Doctoral School of the University of Vienna and the Medical University of Vienna, Vienna, Austria

\# equal contribution

* Corresponding authors

Contact details: david.haselbach@imp.ac.at, andrea.pauli@imp.ac.at

\begin{abstract}
(126 words)
Ribosomes are produced in large quantities during oogenesis and stored in the egg. However, the egg and early embryo are translationally repressed. Using mass-spectrometry and cryo-EM analyses of ribosomes isolated from zebrafish and Xenopus eggs and embryos, we provide molecular evidence that ribosomes transition from a dormant to an active state during the first hours of embryogenesis. Dormant ribosomes are associated with four conserved factors that form two modules and occupy functionally important sites of the ribosome: a Habp4-eEF2 module that stabilizes ribosome levels and a Dap1b/Dapl1-eIF5a module that represses translation. Dap1b/Dapl1 is a newly discovered translational inhibitor that stably inserts into the polypeptide exit tunnel. Thus, a developmentally programmed, conserved ribosome state plays a key role in ribosome storage and translational repression in the egg.
\end{abstract}




\section{Introduction}

Ribosomes are amongst the most abundant macromolecular complexes stored in the quiescent $\operatorname{egg}^{1,2}$. These maternally provided ribosomes (in short: maternal ribosomes) are essential for progression through embryogenesis as they are required for the translation of maternal and zygotic transcripts ${ }^{3-6}$ (Fig. 1a). Although the overall number of ribosomes per embryo remains constant throughout early embryogenesis, prior studies from multiple organisms indicate that translational activity increases in the embryo, suggesting that translation is repressed in the mature egg $^{7-12}$. Several mechanisms, including shortening of mRNA polyadenine tails ${ }^{13}$ and interference with the formation of the translational initiation factor eIF $4 \mathrm{~F}^{14,15}$, have been implicated in dampening translation in the egg and early embryo, while the ribosome itself has not been considered to contribute to this regulation.

Translationally inactive ribosomes have been observed in pro- and eukaryotes in specific cellular contexts, e.g. immunity, viral infection, and nutrient deprivation, and are associated with factors such as Bac $7^{16}$, Nsp $1^{17}$, and Stm $1^{18}$, respectively. Studies from the 1970s with sea urchin eggs indicated the presence of inhibitory proteins which were thought to be associated with maternal ribosomes ${ }^{19-21}$, yet the relevance and molecular identity of these factors remained unclear. How ribosomes are stored in an inactive state for extended amounts of time in the mature egg, and whether regulation of the ribosome itself contributes to translational repression in the egg and the subsequent increase in translational activity during embryogenesis remains thus unknown.

\section{Results}

To investigate the timing of translational activation during the egg-to-embryo transition in vertebrates, we examined the translational status of zebrafish embryos at different stages of development (Fig. 1a). Specifically, we used polysome gradients to uncover the fraction of ribosomes present as individual subunits (40S and 60S), monosomes (80S) and polysomes. In the absence of triggers of ribosome stalling, an increase in the polysome fraction generally indicates increased active translation ${ }^{22}$. We found that ribosomes in the egg and in the 1-hour post fertilization (hpf) embryo were almost exclusively present as monosomes, whereas the polysome fraction started to increase from 3-6 hpf onwards (Fig. 1b, c). As an orthogonal approach, we calculated translational efficiency (TE) values based on ribosome-protected mRNA fragments over the course of zebrafish embryogenesis, using published ribosome profiling and RNA-Seq data sets ${ }^{23,24}$. We observed that translational efficiency increases over the course of embryogenesis, with a clear increase at $6 \mathrm{hpf}$ (Fig. 1d). Together, these data suggest that, similarly to other vertebrates ${ }^{7-12}$, most ribosomes present in the zebrafish egg and early embryo were not engaged in active translation. Given that zygotic ribosomes do not accumulate until after 6 hpf (Fig. 1a) $)^{3,4,25}$, we hypothesize that maternal ribosomes transition from a "dormant" to an "active" state, leading to increased translational activity during the first hours of embryogenesis in zebrafish. 
bioRxiv preprint doi: https://doi.org/10.1101/2021.11.03.467131; this version posted November 3,2021 . The copyright holder for this

preprint (which was not certified by peer review) is the author/funder, who has granted bioRxiv a license to display the preprint in perpetuity. It is made available under aCC-BY-NC 4.0 International license.

\section{Figure 1}
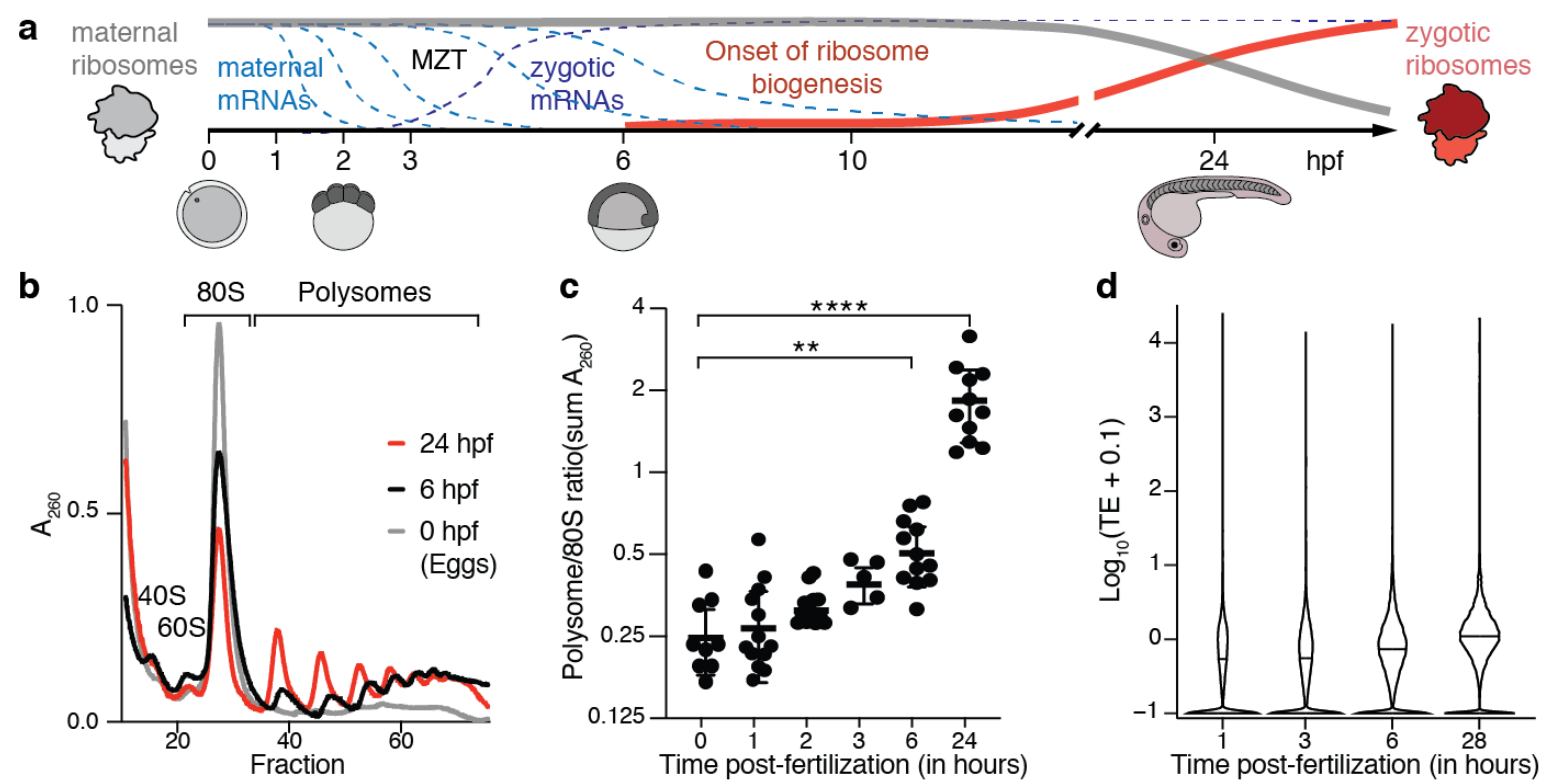

d
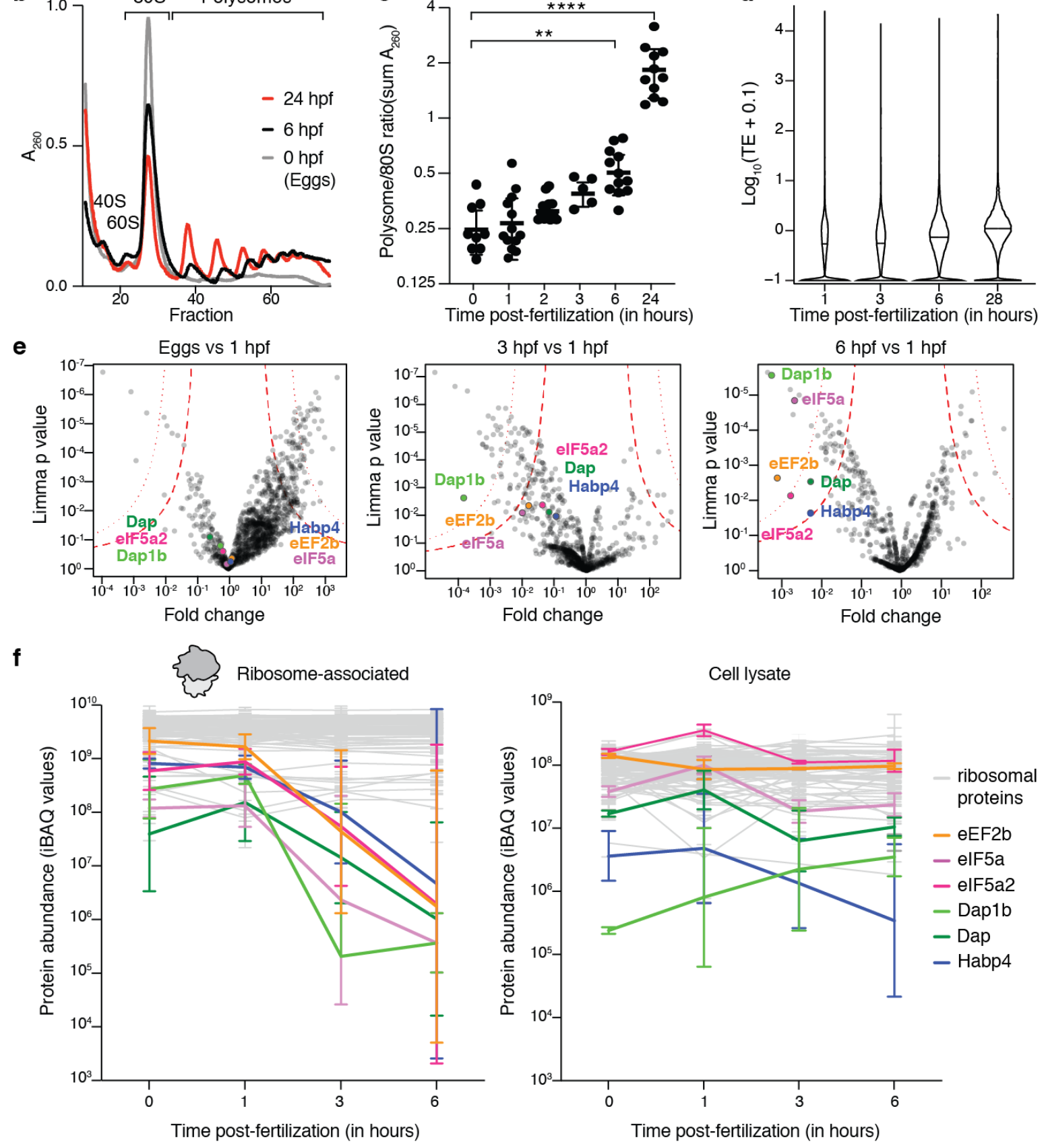

Fig. 1. Increase of translation during zebrafish embryogenesis correlates negatively with the presence of ribosome-bound factors. a, Schematic of the maternal-to-zygotic transition (MZT) in zebrafish, in which the clearance of maternal mRNAs (light blue dashed line) is coordinated with the activation of zygotic mRNA transcription (dark blue dashed line) during the first hours of development. The absolute time (in hours postfertilization (hpf)) is indicated below the graph, along with schematics of embryos. The replacement of maternal ribosomes (grey) by zygotic ribosomes (red) is delayed compared to the MZT and occurs over the course of several days. b, Representative polysome profiles of zebrafish eggs, 6 hpf embryos and 24 hpf larvae. A 260 , absorbance value at $260 \mathrm{~nm}$. c, Quantification of polysome-to-monosome ratios at different stages of development 
(0 h: $\mathrm{n}=8 ; 1 \mathrm{~h}: \mathrm{n}=12 ; 2 \mathrm{~h}: \mathrm{n}=5,3 \mathrm{~h}: \mathrm{n}=6 ; 6 \mathrm{~h}: \mathrm{n}=12 ; 24 \mathrm{~h}: \mathrm{n}=11)(* *: p$-value $=0.0013, * * *: p$-value $>0.0001) . \mathbf{d}$, Violin plots showing the distribution of the median translation efficiency (TE), calculated as the ratio between ribosome protected fragments and total RNA counts, at different developmental time points. e, Volcano plots based on mass spectrometry analysis showing fold enrichments of zebrafish proteins in the ribosome fraction of 1 hpf zebrafish embryos compared to eggs (left), 3 hpf embryos (middle) and 6 hpf embryos (right) (n=3). Permutation-based false discovery rates (FDRs) are displayed as dotted (FDR < 0.01) and dashed (FDR $<0.05)$ lines. Ribosome-associated factors that are stable in eggs and $1 \mathrm{hpf}$ stages but depleted from ribosomes during the first 6 hours of embryogenesis are highlighted in color. f, Abundance changes of a subset of factors relative to core ribosomal proteins in the ribosome-associated proteome (left) and total cell lysate (right) during the egg-toembryo transition $(n=3)$. Protein abundances are reported as iBAQ values (sum of all peptide intensities divided by the number of observable peptides for a given protein). Core ribosomal proteins are plotted in light grey (76/81: left; 74/81: right); ribosome-associated factors that are released from the ribosome are highlighted in color.

To uncover factors that regulate maternal ribosome activity during the first hours after fertilization, we purified bulk ribosomes (monosomes and polysomes) from zebrafish eggs and developing embryos for mass-spectrometry (MS) analysis. While the relative abundances of core ribosomal proteins were similar at all time points, we identified four evolutionarily conserved factors, namely eIF5a/eIF5a2, eEF2b, Habp4 and the paralogs Dap1b/Dap, that were specifically associated with ribosomes in zebrafish eggs and $1 \mathrm{hpf}$ embryos, but depleted from ribosomes in $3 \mathrm{hpf}$ and $6 \mathrm{hpf}$ embryos (Fig. 1e, f). The levels of these ribosome-associated factors remained relatively stable in total embryo lysates over the first 6 hours of development (Fig. 1f), suggesting that they were released from the ribosome but not immediately degraded. Importantly, we found that the same set of factors were enriched in ribosomes purified from Xenopus eggs versus 24 hpf embryos (a developmental stage equivalent to zebrafish $6 \mathrm{hpf}$ embryos) (Extended Data Fig. 1a). Thus, the association of these four factors with ribosomes in the mature egg is conserved in zebrafish and Xenopus and correlates with suppressed translation.

To elucidate how these factors establish a translationally repressed ribosome state, we determined the structures of maternal ribosomes isolated from $1 \mathrm{hpf}$ zebrafish embryos (Fig. 2a) and Xenopus eggs (Fig. 2b) by electron cryo-microscopy (cryo-EM) at 3.2 and $2.8 \AA$ average resolution, respectively (Extended Data Table 1, Extended Data Figs. 2, 3). We were able to assign densities for all four factors in ribosomes from both zebrafish and Xenopus (Fig. 2c, Extended Data Fig. 1b-e,). In parallel, we performed cryo-EM analysis of ribosomes isolated from 6 hpf zebrafish embryos (2.6 ̊̊ average resolution; Extended Data Figs. 1f, 4, Extended Data Table 1). In agreement with the MS data, maternal ribosomes from $6 \mathrm{hpf}$ zebrafish embryos showed low density levels for eEF2b and no densities for eIF5a/eIF5a2, Habp4 and Dap1b/Dap (Extended Data Fig. 1f, Extended Data Table 2).

eIF5a promotes translational elongation and termination, particularly upon ribosome stalling at specific amino acid sequence contexts ${ }^{26,27}$. We found that eIF5a/eIF5a2 binds between the exit (E) and peptidyl (P) site of the ribosome, as previously reported ${ }^{28}$. eEF2 mediates ribosomal translocation ${ }^{29,30}$ and transiently interacts with the tRNA-mRNA complex at the aminoacyl (A) site of the ribosome. Proteins of the Habp4 family (PFAM family PF04774), but not Habp4 itself, have been reported to stabilize the eEF2 interaction at the A site in inactive ribosomes ${ }^{31,32}$. Indeed, we found Habp4 bound to the mRNA entry channel of zebrafish $1 \mathrm{hpf}$ and Xenopus egg ribosomes at the same position as previously reported for its mammalian 
bioRxiv preprint doi: https://doi.org/10.1101/2021.11.03.467131; this version posted November 3, 2021. The copyright holder for this

preprint (which was not certified by peer review) is the author/funder, who has granted bioRxiv a license to display the preprint in perpetuity. It is made available under aCC-BY-NC 4.0 International license.

\section{Figure 2}

a

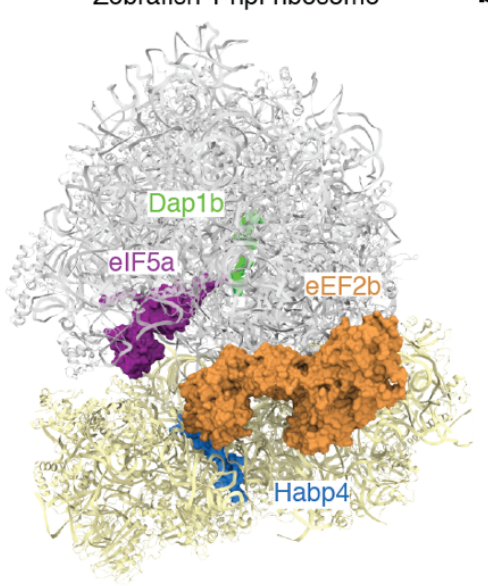

b

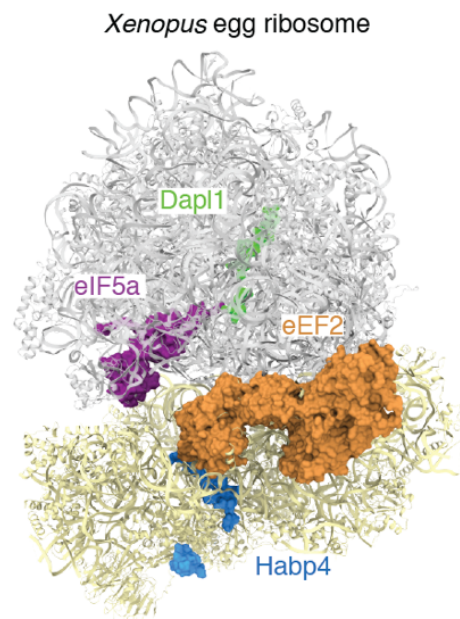

d Zebrafish $1 \mathrm{hpf}$ ribosome

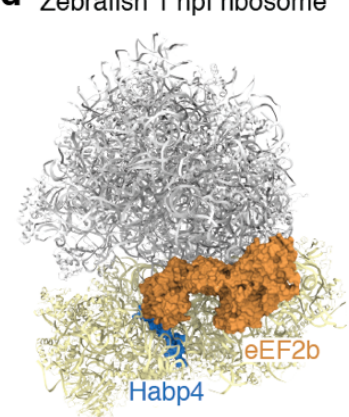

f
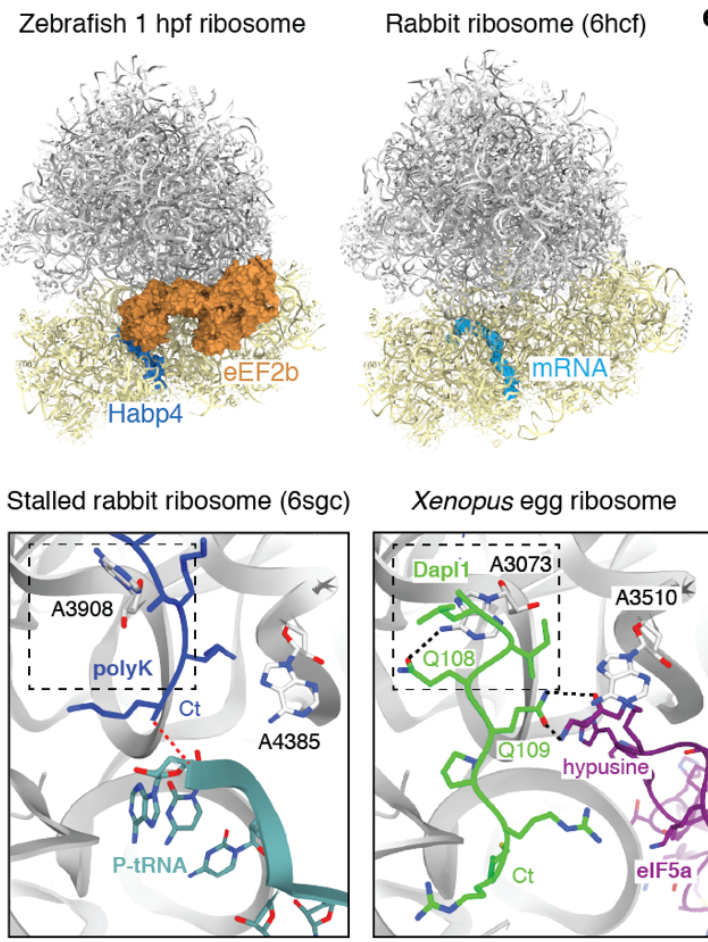

e Zebrafish $1 \mathrm{hpf}$ ribosome
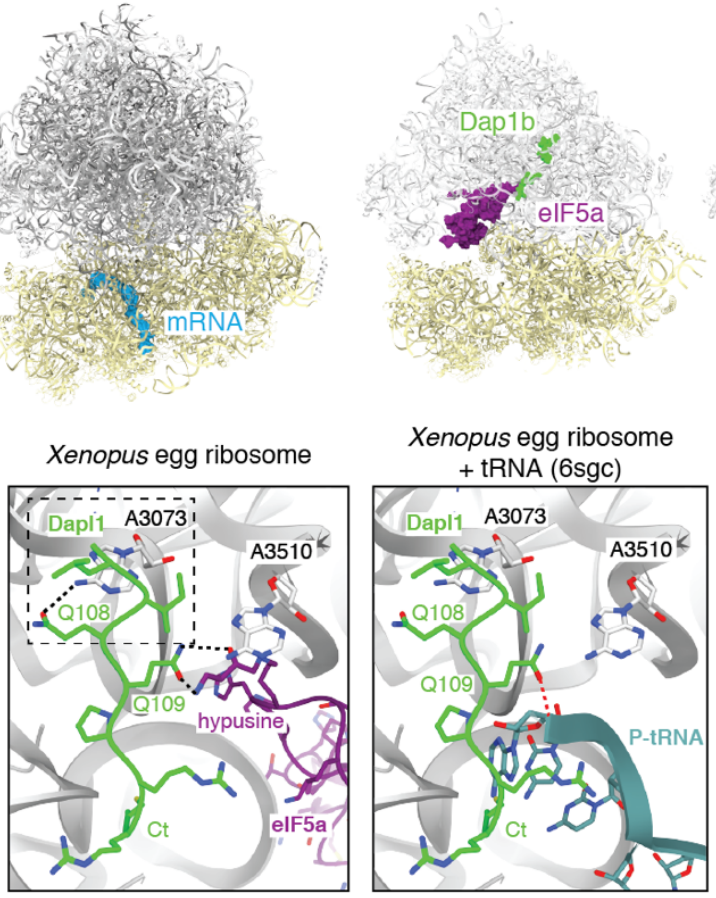

g

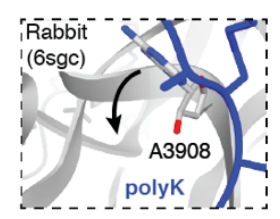

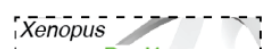

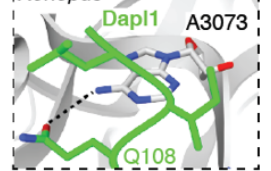

h

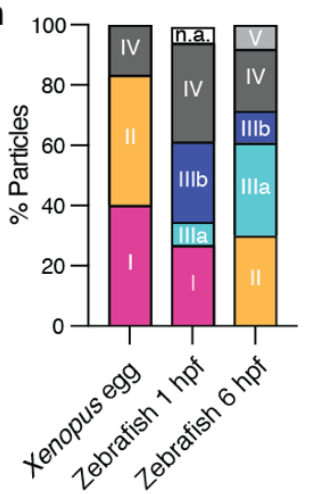

(II)

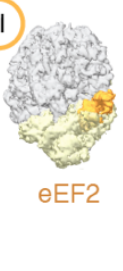

(III)

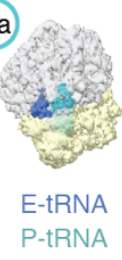

(III)

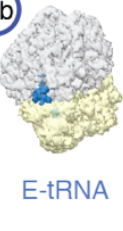

(iv)

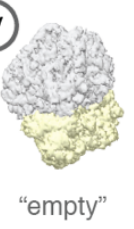

v)

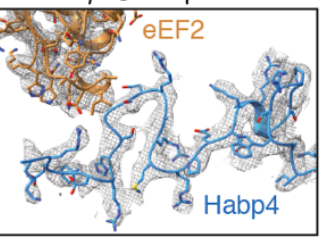

Human ribosome (6ole)

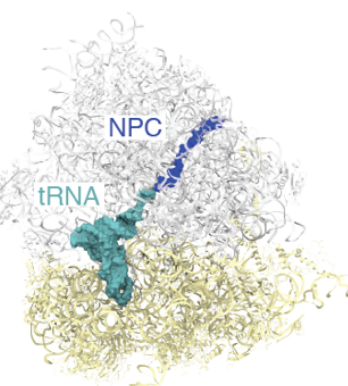

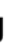

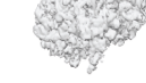

$60 \mathrm{~s}$

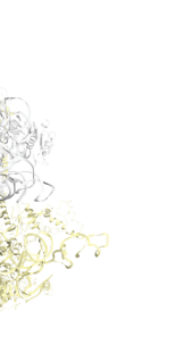

Fig. 2. A conserved set of factors blocks functionally important sites of the egg-state ribosome. a-b, Overview of the dormant ribosome structure from $1 \mathrm{hpf}$ zebrafish (a) and Xenopus egg (b). Core ribosomal proteins and rRNA molecules are shown in gray (large subunit) and yellow (small subunit). Ribosome-associated factors are shown as surface representations; eEF2b (in orange) and eIF5a (in dark magenta) correspond to PDB-6MTE ${ }^{31}$ and PDB-5DAT, and were aligned to ribosomes using the command mmaker in ChimeraX (root-mean-square 
deviations (RMSDs) for alignment to $1 \mathrm{hpf}$ zebrafish ribosome: $0.454 \AA$ and $0.643 \AA$, respectively; RMSDs for alignment to Xenopus egg ribosome: $0.489 \AA$ and $0.642 \AA$, respectively). c, Density maps (in mesh) of the two identified modules of the Xenopus dormant ribosome: Dapl1-eIF5a (top) and Habp4-eEF2 (bottom). The distance between the carboxyl group of Q109 of Dapl1 and the amine group of hypusine-51 of eIF5a is $2.9 \AA$ ( $2.5 \AA$ in the zebrafish $1 \mathrm{hpf}$ ribosome between Q105 of Dap1b and hypusine-51). Ct, C-terminus. d, Habp4 (in blue) and eEF2b (in orange, from $6 \mathrm{MTE}^{31}$ ) are shown as surface representations in the dormant ribosome from $1 \mathrm{hpf}$ zebrafish (left). The structure of the rabbit ribosome stalled on an mRNA (depicted as surface representation in light blue; $6 \mathrm{HCF}^{42}$ is shown on the right for comparison. Both structures were superimposed using the command mmaker in ChimeraX (RMSD: $0.957 \AA$ ). e, Clipping of the dormant ribosome from 1 hpf zebrafish shows Dap1b (in green) bound within the polypeptide exit tunnel (PET) (left). The structure of a stalled human ribosome, $6 \mathrm{OLE}^{43}$, containing a nascent polypeptide chain (NPC, in blue) and a P-tRNA (in turquoise) is shown on the right for comparison. Dap1b, eIF5a (in dark magenta; from 5DAT), the NPC and the P-tRNA are depicted as surface representations. Both structures were superimposed using the command mmaker in ChimeraX (RMSD: 0.689 A). f. Comparison of the peptidyl-transferase center (PTC) of a rabbit $80 \mathrm{~S}$ ribosome stalled with a poly-Lysine NPC (in blue) $\left(6 \mathrm{SGC}^{39}\right.$, left) and of the Xenopus egg ribosome. The large subunits of both models were superimposed using the command mmaker in ChimeraX (RMSD: 0.297 $)$ ). The P-tRNA (in turquoise) (6SGC ${ }^{39}$ ) is shown superimposed onto the Xenopus egg ribosome for comparison on the right (eIF5A is hidden). Critical amino acids and 28S rRNA nucleotides are labelled; interactions are depicted with dashed lines. Boxed areas (dashed boxes) are shown at higher magnification in $\mathrm{g}$. $\mathbf{g}$, Magnified view of the boxed areas in F. A3073 of Xenopus 28S rRNA (bottom, equivalent to A3908 of rabbit $28 \mathrm{~S}$ on top) displays a different conformation when interacting with Gln108 of Dapl1. h, Distribution of ribosomal particles among the classes obtained after the analysis of particle heterogeneity in Xenopus egg ribosomes and zebrafish $1 \mathrm{hpf}$ and $6 \mathrm{hpf}$ ribosomes with cryoDRGN ${ }^{44}$. Representative filtered density maps of the six major classes are shown on the right (see Extended Data Table 2; n.a refers to "non-assigned" particles).

homolog SERBP $1^{31,33}$, sequestering eEF2 at the A site and blocking the interaction sites of the tRNA-mRNA complex on the ribosome (Fig. 2d; Extended Data Fig. 1b, c).

Death Associated Protein 1b (Dap1b)/Dapl1 (in Xenopus Dapl1.L/Dapl1.S; in mammals also known as EEDA (early epithelial differentiation-associated) ${ }^{34,35}$ ) and Dap are highly conserved proteins across animals (Extended Data Fig. 5) but have not been reported to associate with the ribosome. In mammals, Dapl1 has been linked to epithelial differentiation and proliferation ${ }^{34-}$ ${ }^{36}$, and Dap has been described as a positive regulator of programmed cell death ${ }^{37}$ and a negative regulator of autophagy ${ }^{38}$. However, the molecular mechanisms of action for both factors are still unknown. Strikingly, we found density in the polypeptide exit tunnel (PET) of zebrafish $1 \mathrm{hpf}$ and Xenopus egg ribosomes that we could attribute to the conserved C-terminus of Dap1b/Dap11 or Dap (Fig. 2e, Extended Data Figs. 1d, e, 5). Dap1b was $\sim 10$-fold more abundant than Dap in ribosomes from 1 hpf zebrafish embryos according to our MS analysis (Fig. 1f), and only Dapl1 was identified by MS in ribosomes from Xenopus eggs (Extended Data Fig. 1a). Thus, we modelled the C-terminus of zebrafish Dap1b and Xenopus Dapl1, which we found to extend by five amino acids beyond the position that is occupied by the Cterminal amino acid residue of a nascent polypeptide chain (Fig. 2c, f). Intriguingly, an invariant Glutamine in Dap1b/Dapl1 (Gln109 in Xenopus, Gln105 in zebrafish, Extended Data Fig. 5) occupies the same position as the C-terminal residue of a nascent peptide chain ${ }^{39}$ and forms a hydrogen bond with the hypusine residue of eIF5a (Fig. 2f). Of note, hypusination of eIF5a's Lysine residue is essential for eIF5a function ${ }^{40,41}$. Moreover, we observe additional hydrogen-bond interactions between the immediately adjacent Glutamine in Dap1b/Dapl1 (Gln108 in Xenopus, Gln104 in zebrafish, Extended Data Fig. 5) with the 28S rRNA (Fig. 2f, g). Interestingly, the interaction of Xenopus Gln108 (Gln104 in zebrafish) with adenine 3073 
(A3168 in zebrafish) restricts this adenine to a conformation distinct from previously reported ribosomal structures (Fig. 2g). Together, our structural data suggests that Dap1b/Dapl1's specific interactions with eIF5a and the 28S rRNA contribute to Dap1b/Dapl1's binding to the PET of a fully assembled eukaryotic $80 \mathrm{~S}$ ribosome, thus making them unique among all ribosome-associated factors characterized so far.

To determine whether the four factors are bound to the same ribosomal particles, we performed a 3D variability analysis after dimensional reduction of the datasets using cryoDRGN ${ }^{44}$. Each dataset's latent representation showed distinct clusters when visualized in UMAPs (Extended Data Fig. 1g, h). A detailed analysis of the particles in these clusters revealed six major classes based on the presence or absence of eEF2, eIF5a, and tRNAs (Extended Data Table 2). In contrast to previous reports suggesting mutually exclusive binding of eIF5a and eEF2 to the ribosome ${ }^{26,31,45}$, our analysis revealed that $40.2 \%$ and $26.9 \%$ of ribosomes in Xenopus and in 1 hpf zebrafish, respectively, were simultaneously bound by eIF5a and eEF2 (Fig. 2h, class I particles). Due to the dimensional reduction of the datasets, cryoDRGN-derived maps were not of sufficient resolution to confidently assess the presence of Dap1b/Dap and Habp4 in any of the clusters. However, given the data from the literature on Habp4 homologs binding to eEF2containing ribosomes ${ }^{31-33}$ and our data showing that eIF5a and Dap1b interact with each other (Fig. 2c, f), our results are consistent with Dap1b and Habp4 being bound to class I (eIF5a- and eEF2-containing) ribosomes (Fig. 2h). In support of this conclusion, class I particles were not observed in the translationally more active $6 \mathrm{hpf}$ zebrafish embryos (Fig. 2h, Extended Data Table 2, Extended Data Fig. 1h). In contrast to class I particles, which were exclusively identified in the translationally repressed zebrafish and Xenopus egg states and will thus in the following be referred to as "dormant" state, other ribosomal particle classes were identified to varying levels in all data sets, including in $6 \mathrm{hpf}$ zebrafish embryos (Fig. 2h, Extended Data Fig. 1h). Other particle classes include class II particles that contained only eEF2, class III particles that contained tRNAs, and "empty" class IV particles that lack eEF2, eIF5a, and tRNAs (Fig. 2h). "Empty" class IV particles may correspond to ribosomes which have lost ribosome-associated factors or tRNAs during the purification since they do not cluster together but occupy different positions in the UMAPs (Extended Data Fig. 1h). Together, our results support a novel, dormant ribosome state present in the mature egg in which four functionally important sites of the ribosome, namely the A site and the E/P site, the mRNA channel and the PET are occupied by evolutionarily conserved factors.

While we could assign Cryo-EM densities for small amino acid stretches within Habp4 and Dap1b/Dap1l and Dap proteins, the majority of the polypeptide chains are not visible in our ribosome structures. To determine the path of these proteins in relation to ribosomal proteins, as well as to confirm that Dap1b/Dapl1 and Dap are novel ribosome-associated factors, we performed crosslinking MS with the primary amine crosslinker disuccinimidyl sulfoxide (DSSO), using ribosomes purified from $1 \mathrm{hpf}$ zebrafish embryos and Xenopus eggs (Fig. 3a-d, Extended Data Tables 3-5). We obtained more than 1000 crosslinked peptides for each ribosome sample. 95\% (zebrafish) and 90\% (Xenopus) of all mapped crosslinks had C $\alpha-\mathrm{C} \alpha$ distances below the expected maximum crosslinking length of $23 \AA$ (Extended Data Fig. 1i), revealing high quality of our crosslinking MS data ${ }^{46}$. We found crosslinks of the N-terminus of Habp4, which was not visible in cryo-EM densities, with small and large subunit ribosomal 
bioRxiv preprint doi: https://doi.org/10.1101/2021.11.03.467131; this version posted November 3, 2021. The copyright holder for this

preprint (which was not certified by peer review) is the author/funder, who has granted bioRxiv a license to display the preprint in perpetuity. It is made available under aCC-BY-NC 4.0 International license.

Figure 3

a
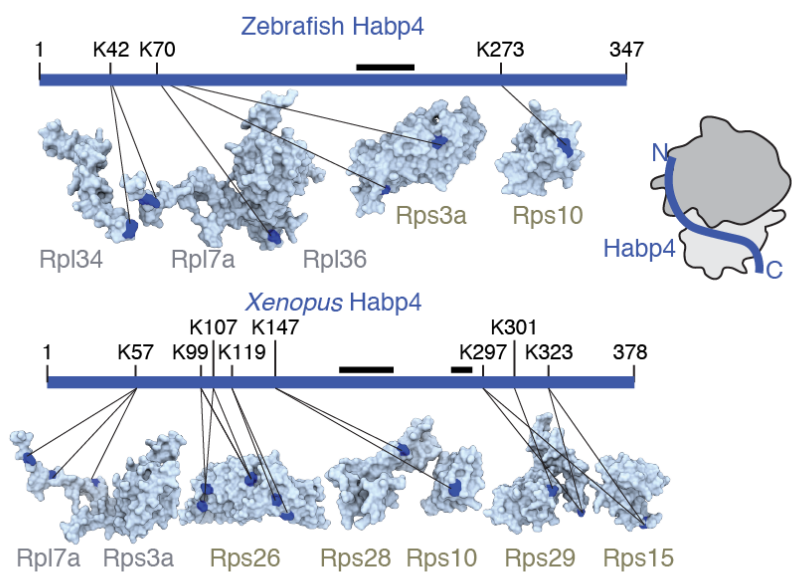

b

Zebrafish $1 \mathrm{hpf}$ ribosome

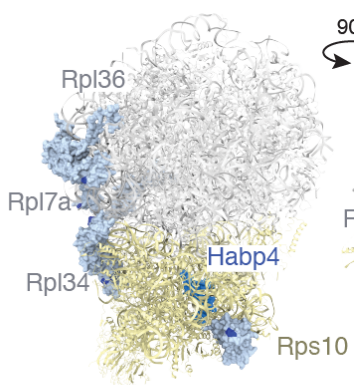

e

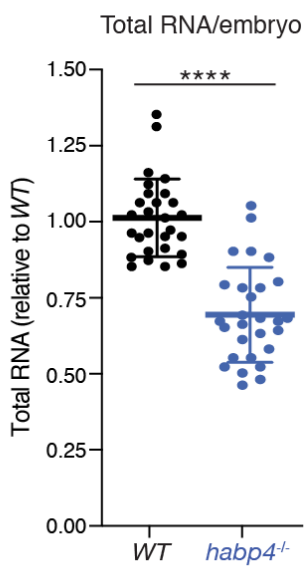

h
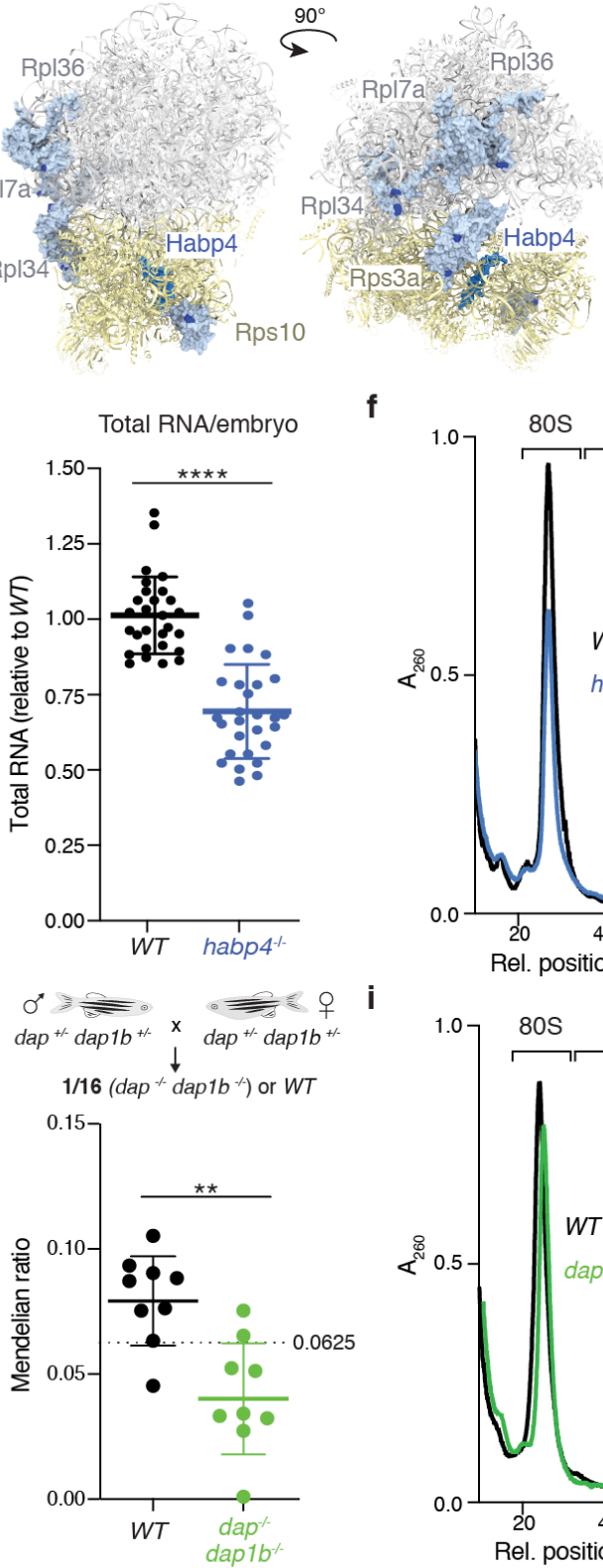

f
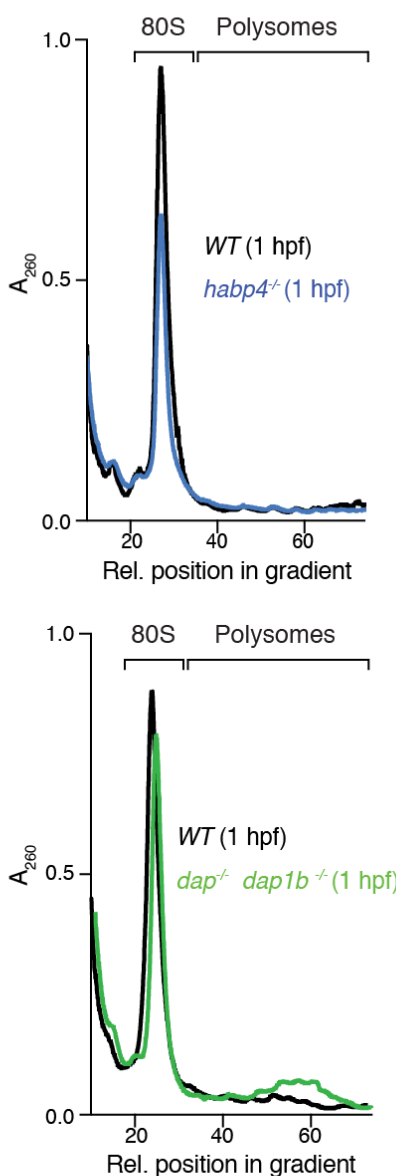

c

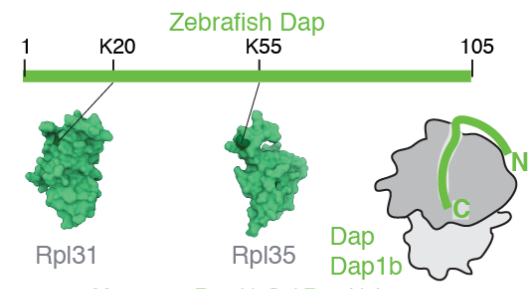

Xenopus Dapl1.S / Dapl1.L

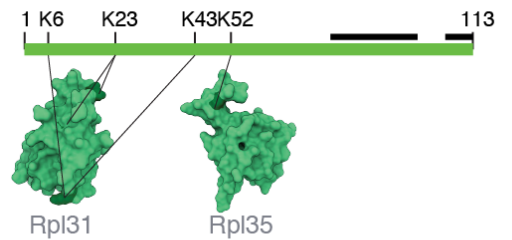

Zebrafish $1 \mathrm{hpf}$ ribosome

d

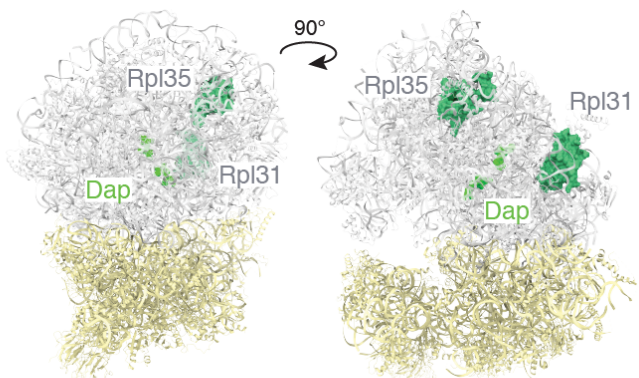

g

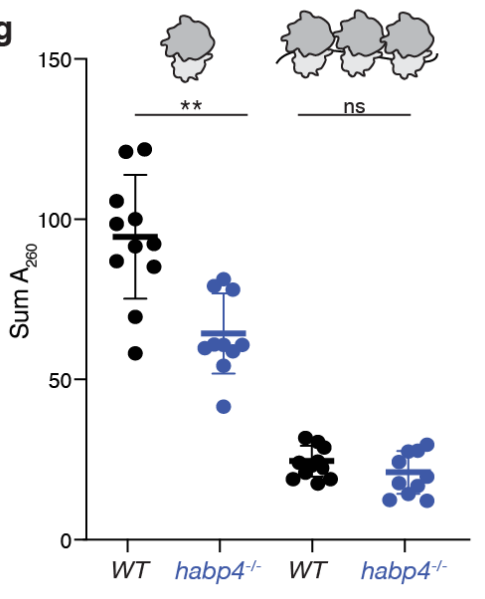

j

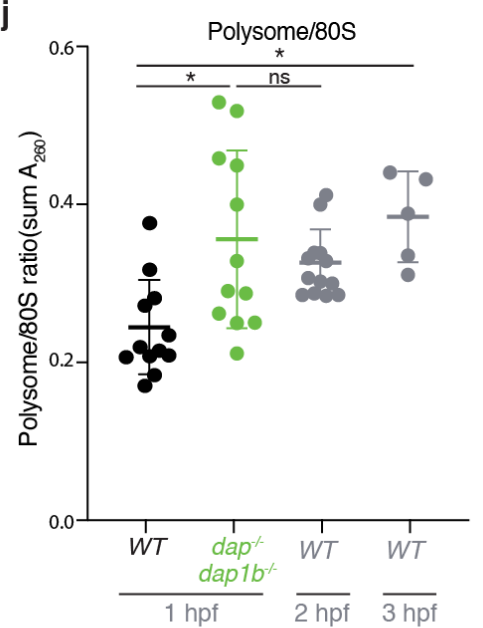

Fig. 3. Habp4 binding to the mRNA channel stabilizes monosomes in the egg, while Dap1b/Dap binding to the polypeptide exit tunnel represses polysome formation in zebrafish embryos. a, Crosslinking mapping of 
Habp4 (depicted as a scheme; modeled regions highlighted with a black line) to proteins of the 1 hpf zebrafish (top) and Xenopus egg (bottom) ribosomes. Crosslinked proteins are shown as surface representations, with crosslinked residues depicted in dark blue. A cartoon on the top right shows the proposed position of Habp4 (in blue) on the ribosome based on crosslinking and cryo-EM data. b, Position of the proteins crosslinked to zebrafish Habp4 (shown as surface representations) in the $1 \mathrm{hpf}$ zebrafish ribosome. c, Crosslinking mapping of Dap and Dapl1 (depicted as a scheme; modeled regions highlighted with a black line) to proteins of the 1 hpf zebrafish (top) and Xenopus egg (bottom) ribosomes, respectively. Crosslinked proteins are shown as surface representations, with crosslinked residues depicted in dark green. A cartoon on the top right shows the proposed position of Dapl1/Dap (in green) on the ribosome based on crosslinking and cryo-EM data. d, Position of the proteins crosslinked to zebrafish Dap (shown as surface representations) in the 1 hpf zebrafish ribosome. e, Comparison of the amount of total RNA per embryo, normalized to the average total RNA amount per wild-type embryo, from 1-3 hpf embryos derived from wild-type $(W T)$ and habp $4^{-/}$parents $(\mathrm{n}=28$, p-value $<0.0001)$. f, Representative polysome profiles from $1 \mathrm{hpf}$ embryos derived from $W T$ and habp ${ }^{-/}$parents. A260, absorbance value at $260 \mathrm{~nm}$. g, Quantification of the monosome (left) and polysome (right) peaks of polysome profiles from 1 hpf embryos derived from $W T$ and habp $4^{-/}$parents $(\mathrm{n}=10$, p-value (monosome comparison) $=0.0056$; ns, not significant). h, Mendelian ratio of fin-clipped adult fish. Adult double homozygous $d a p^{-/}$, dap $\mathrm{b}^{-/-}$fish as well as WT fish are expected at $6.25 \%$ (1/16 fish) from an in-cross of double-heterozygous dap $^{+/-}$, dap $1 b^{+/-}$parents $(\mathrm{n}=9$ crosses; min. 84 fish analyzed per cross; total number of fish genotyped: 1029). i, Representative polysome profiles from $1 \mathrm{hpf}$ embryos derived from $W T$ and $d a p^{-/}$, dap $1 b^{-/-}$parents. j, Quantification of polysome-tomonosome ratios of polysome profiles from $1 \mathrm{hpf}$ embryos derived from $W T$ and dap $^{-/}$, dap $1 b^{-/-}$parents $(\mathrm{n}=12$, p-value [1 hpf WT vs 1 hpf dap ${ }^{-/}$, dapl $\left.1 b^{-/}\right]=0.0156$; p-value [2 hpf $W T$ vs 1 hpf dap ${ }^{-/}$, dap $\left.1 b^{-/}\right]=$ns, not significant; $p$-value $[1 \mathrm{hpf} W T$ vs $3 \mathrm{hpf} W T]=0.0047)$.

proteins, including Rpl7a and Rps3a, in dormant ribosomes from both zebrafish and Xenopus (Fig. 3a, b). Crosslinking MS analyses of Dap1b/Dapl1 and Dap showed that the N-terminal regions are proximal to the polypeptide exit site, which is consistent with insertion of their $\mathrm{C}$ termini into the PET. In particular, we found crosslinks between a highly conserved $\mathrm{N}$-terminal region of Dapl1/Dap and Rpl31, and between the central region of Dapl1/Dap and Rp135 (Fig. 3c, d; Extended Data Fig. 5), indicating that both N- and C-terminal regions of Dap1b/Dapl1 and Dap are in close proximity to the ribosome.

Zebrafish and Xenopus habp4, daplb/dapll and dap mRNAs are highly expressed during oogenesis, whereas eif5a and eef2 transcripts are abundant in all tissues (Extended Data Fig. 6). Moreover, Habp4 family proteins have been linked to ribosome stabilization during nutrient deprivation in yeast $(\mathrm{Stm} 1)^{47}$ and to non-translating ribosomes in yeast ${ }^{47,48}$, Drosophila (Vig2) $^{33}$ and mammals (SERBP1) ${ }^{31,33,49}$. Based on these observations, we hypothesized that Habp4 and Dap1b/Dap11/Dap play key roles in the prolonged ribosomal storage and/or translational repression in the egg. To test this hypothesis, we used CRISPR/Cas9-based mutagenesis to generate habp4 knockout $\left(\right.$ habp $\left.4^{-/}\right)$zebrafish lines (Extended Data Fig. 7a). $\mathrm{Habp4}^{-/}$mutants were viable and showed normal egg clutch sizes, fertility, embryo development and survival rates (Extended Data Fig. 7b-f). However, 1-3 hpf embryos derived from $h a b p 4^{-/}$parents contained about $30 \%$ less total RNA as well as $30 \%$ fewer monosomes when compared to 1-3 hpf wild-type siblings (Fig. 3e-g) despite having a similar average embryo size (Extended Data Fig. $7 \mathrm{~g}$ ). Given that $80-90 \%$ of the total cellular RNA is ribosomal $\mathrm{RNA}^{50}$, the reduction in total RNA content in habp4 $4^{-/}$embryos is likely due to the loss of rRNA-containing ribosomes. In contrast, embryos from $h a b p 4^{-/}$and from wild-type parents displayed no significant difference in polysome levels at $1 \mathrm{hpf}$ (Fig. 3g; Extended Data Fig. 7h), suggesting that Habp4 contributes to stabilizing 80S ribosomes but not to inhibiting translation in the zebrafish egg. 
To analyze the physiological function of Dap1b/Dapl1 and Dap, we generated double mutant zebrafish lacking both Dap1b and Dap proteins $\left(\right.$ dap $^{-/}$, dap $\left.1 b^{-/}\right)$(Extended Data Fig. 7i). Adult $\mathrm{dap}^{-/}$, dap $1 \mathrm{~b}^{-/}$mutant fish were obtained from a double heterozygous parent in-cross $\left(\right.$dap $^{+/}$, dap $1 b^{+-}$) at sub-Mendelian ratio compared to wild-type siblings (Fig. 3h), indicating a fitness defect. Though surviving $d a p^{-/}$, dap $1 b^{-/}$adults appeared morphologically normal and showed normal egg clutch sizes, fertility, and embryo development and survival rates (Extended Data Fig. $7 \mathrm{j}-\mathrm{m}$ ), their progeny displayed a significantly higher polysome-to-monosome ratio at $1 \mathrm{hpf}$ compared to $1 \mathrm{hpf}$ embryos from wild-type fish (Fig. 3i, j; Extended Data Fig. 7n, o). Indeed, this ratio was similar to that observed for $2 \mathrm{hpf}$ and $3 \mathrm{hpf}$ embryos from wild-type parents (Fig. $3 \mathrm{j}$ ), suggesting that Dap1b/Dap function as a translational inhibitor in dormant ribosomes of eggs and early embryos.

Dap1b/Dapl1 and Dap are small $(\sim 15 \mathrm{kDa})$, unstructured proteins that are rich in basic amino acids and prolines (15\%) (Fig. 4a; Extended Data Fig. 5). While invertebrates contain only one ortholog, vertebrates have evolved two paralogs (Fig. 4a, Extended Data Fig. 5). Despite displaying less than $50 \%$ overall sequence identity, all Dap1b/Dapl1 and Dap proteins share two highly conserved motifs at the N- (P-A-V-K-A-G-G-M/K-R) and C-terminus (I-Q/H-QP-R-K/R-x), which we found proximal to Rpl31 (Fig. 3c, d) and binding to the PET (Fig. 2ac), respectively (Fig. 4a, Extended Data Fig. 5). To further investigate Dap1b/Dapl1's and Dap's function, we performed in vitro translation assays in rabbit reticulocyte lysate (RRL) in the presence of recombinant Dap1b/Dap proteins (Fig. 4b). Whereas recombinant zebrafish Dap or the negative control BSA did not affect the translational activity of RRL, recombinant zebrafish Dap1b repressed the translation of a luciferase mRNA (IC50: 4.7 $\mu \mathrm{M}$ ) to a similar extent as the antimicrobial peptide Bac7 (IC50: $3.6 \mu \mathrm{M}$ ) (Fig. 4c), which binds to the PET and inhibits translation ${ }^{16}$. To investigate whether the observed difference in repressive activity between zebrafish Dap1b and Dap was generalizable to other species' Dap1b/Dap11 and Dap proteins, or whether it reflected a paralogue-specific mismatch between zebrafish Dap protein and mammalian ribosomes, we assessed ribosomal binding of in vitro translated flag-tagged dap 1b/dapll and dap mRNAs from various species (Fig. 4b). Consistent with a difference in repressive activity between Daplb and Dap in vitro, Dap showed a weaker affinity for rabbit ribosomes than Dap1b/Dapl1 from three different vertebrate species (Fig. 4d, e; Extended Data Fig. 8a). Analysis of ancestral Dap1 proteins from invertebrates revealed that Dap1 from $C$. elegans and from the coral Pocillopora had an affinity for rabbit ribosomes in between that observed for vertebrate Dap and Dap1b proteins, while Dap from D. melanogaster did not bind to rabbit ribosomes (Fig. 4d, e; Extended Data Fig. 8a). Interestingly, we noticed that all rabbit ribosome-binding vertebrate Dap1b/Dapl1 proteins as well as the ancestral Pocillopora and $C$. elegans Dap1 proteins contain at least one additional residue at the C-terminus compared to non-binding vertebrate and Drosophila Dap proteins (Fig. 4a; Extended Data Fig. 5). However, extending zebrafish Dap or shortening zebrafish Dap1b by one amino acid did not have any significant effect on ribosome binding (Fig. 4f, g; Extended Data Fig. 8b). In contrast, mutating the conserved, hypusine-interacting Gln105 of zebrafish Dap1b (Fig. 2f) (as well as the corresponding Gln102 of zebrafish Dap) abolished binding of Dap1b (and Dap) to the ribosome (Fig. 4f, g; Extended Data Fig. 8b), thus establishing a key function of this invariant Glutamine in stabilizing Daplb binding to the ribosome via its interaction with eIF5A. Together, these 
bioRxiv preprint doi: https://doi.org/10.1101/2021.11.03.467131; this version posted November 3, 2021. The copyright holder for this

preprint (which was not certified by peer review) is the author/funder, who has granted bioRxiv a license to display the preprint in perpetuity. It is made available under aCC-BY-NC 4.0 International license.

\section{Figure 4}

a

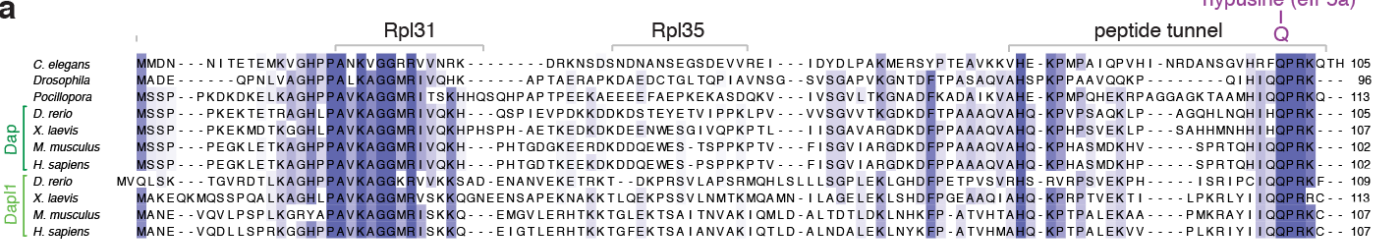

b
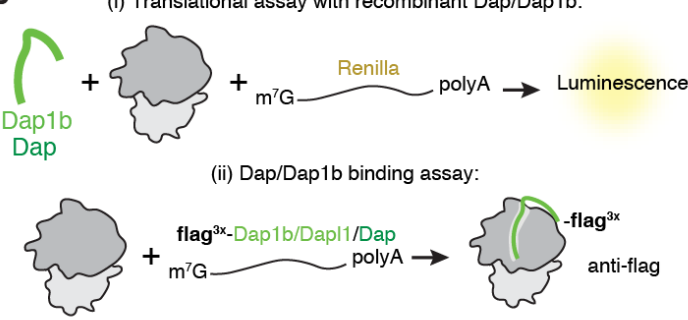

d

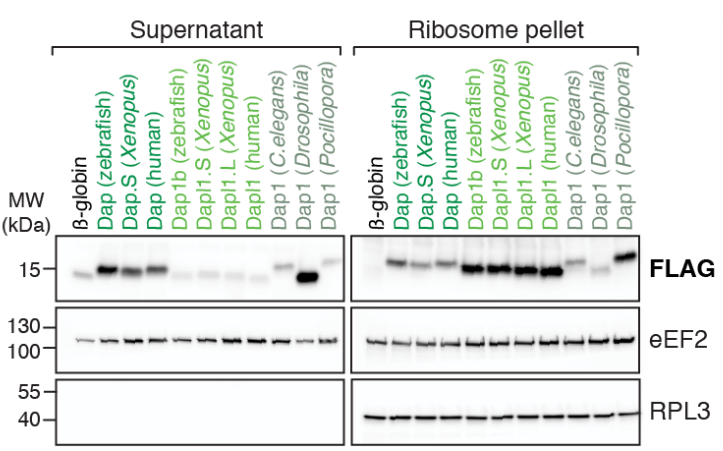

f

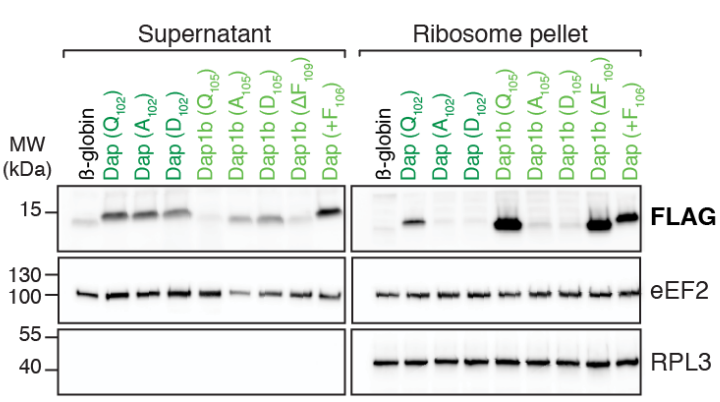

h

Rabbit ribosome with recombinant Dap1b

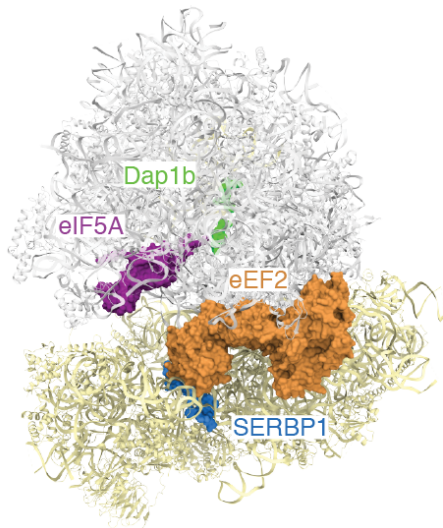

i
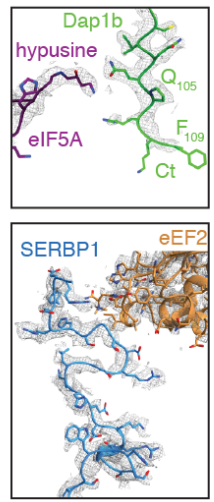

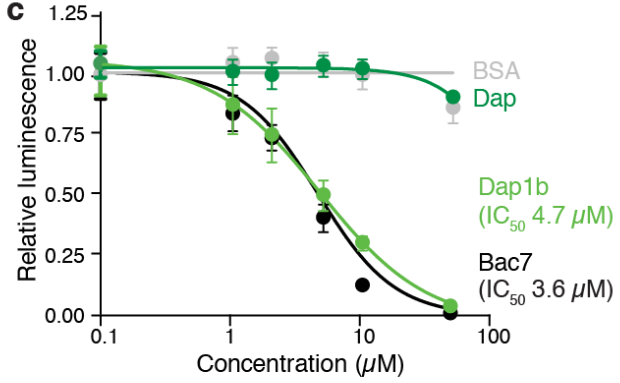

e

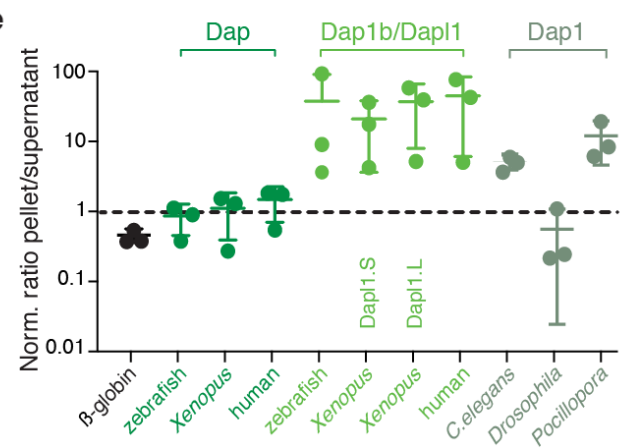

g

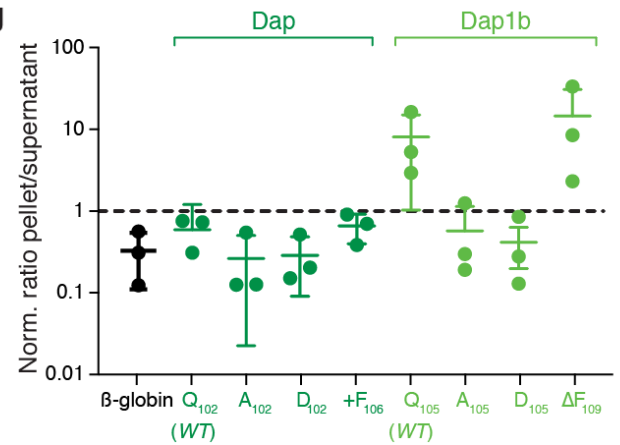

(1)

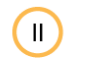

(III)

(III)

(IV)

(v)

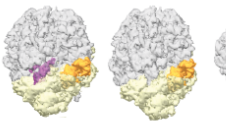

$\begin{array}{llllll}\text { elF5A } & \text { eEF2 } & \text { E-trNA } & \text { E-trNA } & \text { "empty" } & \text { as } \\ \text { eEF2 } & & & \end{array}$

"dormant"

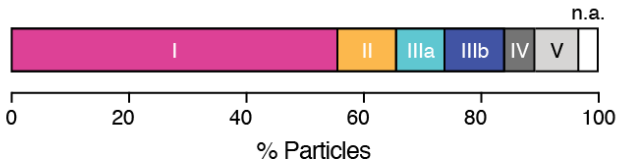

j
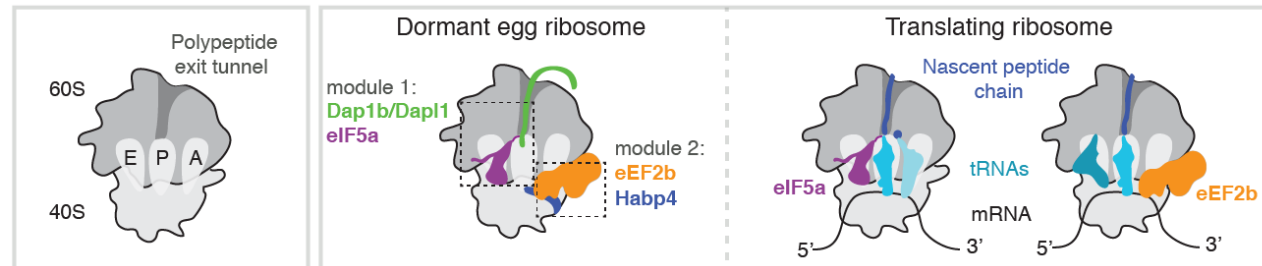
Fig. 4. Dap1b/Dapl1 binding to mammalian ribosomes blocks translation and reconstitutes the egg-like ribosome-state in vitro. a, Alignment of Dap1b/Dapll (light green) and Dap (dark green) paralogs as well as ancestral Dap1 proteins from a subset of vertebrate and invertebrate species. Conserved regions are highlighted in shades of blue according to sequence identity (dark blue represents high concordance). A more extended alignment is shown in Extended Data Fig. 5a. b, Scheme of the in vitro translation assays performed in rabbit reticulocyte lysate (RRL). To investigate Dap1b/Dap activity (top), recombinant zebrafish Dap1b or Dap (or control proteins) and in vitro synthesized Renilla luciferase mRNA were added to RRL. Translation was assessed after 1.5 hours by measuring Luciferase activity. To assess Dap1b/Dapl1 and Dap binding to the ribosome (bottom), in vitro synthesized mRNAs encoding N-terminally tagged candidate proteins were incubated in RRL. Ribosomes were pelleted and Dap1b/Dapl1 and Dap levels were quantified by Western blot. c, IC50 analyses of the sufficiency of recombinant zebrafish Dap1b and Dap proteins to repress translation in RRL (see Fig. 4b, top). The antimicrobial peptide Bac7 and BSA were used as controls. For statistical analysis, four parameter logistic curve (best-fit solution, nonlinear regression-dynamic fitting) and normality tests (Kolmogorov-Smirnov) were used. d, Binding assays of in vitro translated proteins to rabbit ribosomes (see Fig. 4b, bottom). Western blots of ribosomal and supernatant fractions from binding assays with Flag-tagged $\beta$-globin (control) and Dap, Dap1b/Dap11 and ancestral Dap1 from different species. eEF2 and RPL3 levels are used as readout of successful fractionation and for normalization. Blot of the total reaction is shown in Extended Data Fig. 8a. e, Quantification of Western blot signals detected in ribosome pellets compared to supernatants. Values are normalized to loading (eEF2/RPL3) and translation efficiency (FLAG signal of total lysate; Extended Data Fig. 8a) of the individual Flag-tagged factor mRNAs $(n=3)$. $\mathbf{f}$, Western blots of ribosomal and supernatant fractions from binding assays using Flag-tagged $\beta$-globin (control), as well as wild-type and mutant versions of zebrafish Dap and Daplb. Mutant versions are indicated. Blot of the total reaction is shown in Extended Data Fig. 8b. g, Quantification of Western blot signals detected in ribosome pellets compared to supernatants. Values are normalized to loading (eEF2/RPL3) and translation efficiency (FLAG signal of total lysate; Extended Data Fig. 8b) of the individual Flag-tagged factor mRNAs $(n=3)$. h, Structure of the ribosome from RRL supplemented with recombinant zebrafish Dap1b. Ribosome-associated factors are shown as surface representations. Densities (in mesh) for the two modules characteristic of dormant ribosomes (Dap1b-eIF5A and SERBP1-eEF2) are shown on the right. Critical amino acids are indicated. Ct, C-terminus. i, Distribution of ribosomal particles among the classes obtained after the analysis of particle heterogeneity in rabbit ribosomes supplemented with zebrafish Daplb using cryoDRGN. Representative filtered density maps of the six major classes are shown on the top (see Extended Data Table 2; n.a refers to "non-assigned" particles). j, Scheme depicting the main features of the dormant ribosomes identified in this study. A cartoon of the important functional sites of the ribosome is shown on the left. Dormant ribosomes (middle cartoon) are associated with four factors which form two modules: the Habp4-eEF2 module occupies the mRNA channel and A site of the ribosome and interferes with mRNA and A-tRNA binding, and the Dap1b/Dapl1-eIF5A module blocks the polypeptide exit tunnel and the E/P sites. Two cartoons of a translating ribosome are shown on the right for comparison.

analyses revealed that Dap1b/Dapl1 but not Dap efficiently binds to mammalian ribosomes in vitro and is sufficient to inhibit translation.

To assess whether Dap1b was inserted into the PET of mammalian ribosomes, and whether Dap1b addition to translational extracts indeed reconstitutes the dormant ribosome state, we performed cryo-EM on ribosomes isolated from an in vitro translation extract supplemented with $20 \mu \mathrm{M}$ of recombinant zebrafish Dap1b and obtained a map at $2.3 \AA$ average resolution (Fig. 4h; Extended Data Fig. 9, Extended Data Table 1). Notably, we were able to assign densities, not only for Dap1b in the PET (Fig. 4h, Extended Data Fig. 8c), but also for the other dormant ribosome factors, namely eIF5A, eEF2, and the Habp4 homolog SERBP1 (Fig. 4h, Extended Data Fig. 8c, d), which are present in RRL ${ }^{31}$. Rabbit ribosomes with bound Dap1b revealed additional interactions between eIF5A and RPL10A, which is part of the L1 stalk. We found that this interaction restrains the L1 stalk to a closed conformation, as previously reported for eIF5A-containing ribosomes ${ }^{28}$, which thus links the closed L1-stalk (with a locked E site) to the Dap1b-eIF5A module (Extended Data Fig. 8e, Extended Data Table 2). A 3D classification of these ribosomal particles using cryoDRGN ${ }^{44}$ revealed that, after addition of 
zebrafish Dap1b to RRL, $55.7 \%$ of the particles contained both eIF5A and eEF2 (Fig. 4i (class I), Extended Data Fig. 8f, Extended Data Table 2), similarly to dormant ribosomes from $1 \mathrm{hpf}$ zebrafish embryos and from Xenopus eggs (Fig. 2h, Extended Data Table 2). While several cryo-EM ribosome structures isolated from RRL have been published, none of them has reported the co-existence of eIF5A and eEF2 $2^{30,31}$, which is consistent with Dap1b being causally linked to this dormant ribosome state. Together, our data reveal that zebrafish Dap1b can insert into the PET of mammalian ribosomes, which is sufficient to reconstitute the dormant ribosomal state in vitro and to efficiently block translation.

\section{Discussion}

Here, we show that ribosomes are stored in zebrafish and Xenopus eggs in a newly identified dormant state, which can be reconstituted in mammalian ribosomes in vitro. This state is characterized by the binding of a conserved set of factors that occupy and thereby block functionally important sites of the ribosome (Fig. 4j). Our cryo-EM structures revealed two modules that co-exist on dormant ribosomes. One module is formed by Habp4-eEF2, in which binding of the egg-specific SERBP1-homolog Habp4 to the mRNA channel stabilizes eEF2 at the A site, which blocks mRNA and tRNAs from interacting with the ribosome. In vivo data reveal that Habp4 has a key role in stabilizing ribosomes in the egg (Fig. 3e-g), thus contributing to storing the large numbers of ribosomes that are needed for later embryogenesis. The other module is formed by Dap1b/Dapl1-eIF5a, in which insertion of Dap1b/Dapl1 into the PET mimics an arrested nascent peptide chain in the absence of bound mRNA or tRNAs and induces stable binding of eIF5a at the E/P site of the ribosome (Fig. 2a-c, Fig. 2e-f). Importantly, Dap1b binding to mammalian ribosomes is sufficient to induce the dormant ribosomal state in vitro (Fig. 4h, i) and to repress translational activity in vivo (Fig. 3i, j) and in vitro (Fig. 4c). Thus, the two modules act together to accomplish the specific needs of egg cells to (1) stably store ribosomes for prolonged periods of time, and (2) to repress translation. Importantly, repression of the translational machinery in the egg might also be important for the storage of maternal mRNAs since mRNA degradation has been shown to positively correlate with translation ${ }^{51}$. Moreover, having eIF5a and eEF2 stably bound to maternal ribosomes might thus preserve and protect these factors for their later use in embryogenesis, as eEF2 and eIF5a from C. elegans were shown to be prone to aggregate with aging ${ }^{52}$.

Our in vivo data suggest that Habp4, which occupies the mRNA entry channel, is important to preserve maternal ribosomes in the egg. Despite the lower rRNA and monosome content observed in early habp $4^{-/}$embryos, they did not display any developmental defects under our standard growth conditions. This observation was surprising given that a reduction in ribosome levels had previously been shown to cause developmental defects (minute phenotypes in Drosophila $^{53}$ ) and to alter cell differentiation (e.g. in embryonic stem cells ${ }^{54}$ and tumor formation in zebrafish ${ }^{55}$ ). We therefore speculate that ribosomes in zebrafish eggs are stored in excess since embryos can tolerate a reduction of about $30 \%$ of ribosomes. Maternal ribosomes could fulfil additional functions beyond protein synthesis, as they have been proposed to contribute to nucleotide homeostasis during animal development ${ }^{56}$. Other organisms with a smaller nutrient reservoir in eggs than zebrafish might be more dependent on the degradation 
of maternal ribosomes to obtain the nucleotides and amino acids necessary for some biosynthetic pathways. Therefore, Habp4 might be essential in organisms whose (i) maternal ribosomes must be stored for long periods of time and/or (ii) eggs do not contain a large nutrient reservoir.

Our study identified Dap1b/Dapll as a novel translational inhibitor bound to the PET of eukaryotic $80 \mathrm{~S}$ ribosomes that is sufficient to reconstitute the egg ribosome state and can effectively block mammalian translation in vitro (Fig. 4c, Fig. 4h, i). This finding is remarkable since no endogenously made eukaryotic protein had been demonstrated before to be inserted into the PET of eukaryotic $80 \mathrm{~S}$ ribosomes, leading to their repression. Several factors, including the antimicrobial peptides Bac $7^{16}$ and Api137 $7^{57}$, have been shown to be produced in eukaryotic cells as a defense against bacteria. They interact with the PET of bacterial ribosomes and repress bacterial translation (Extended Data Fig. 8g, h). In eukaryotes, only factors involved in ribosome biogenesis (e.g. Reh1, Rei1 and Nog1) have been observed to be inserted into the PET, yet not of mature ribosomes but during eukaryotic $60 \mathrm{~S}$ ribosome biogenesis ${ }^{58-61}$ (Extended Data Fig. 8g, h). Given that $d_{a p^{-/}}$, dap $1 b^{-/}$mutants do not show any developmental phenotype during embryogenesis (Extended Data Fig. $7 \mathrm{j}, \mathrm{m}$ ) and that we do not observe an increase in the amount of polysomes in $\mathrm{dap}^{-/}$, dap $1 b^{-/-}$eggs (Extended Data Fig. 7o), we hypothesize that other inhibitory mechanisms, e.g. short polyadenine tails ${ }^{13}$ and inhibition of eIF4F ${ }^{14,15}$, can at least in part compensate for the loss of the dormant ribosomal state in the egg and keep translation low.

Two questions that will require future work is how Dap1b/Dapl1 is inserted into the PET of the ribosome, and how it is released after fertilization. Our in vitro data with recombinant Dap1b protein suggests that Dap1b is inserted into the PET post-translationally (Fig. 4c, h). Given the sequence conservation of the N- and C-terminus of Dap1b/Dapl1 and Dap proteins, and the presence of crosslinks between Dapl1's and Dap's N-terminus with Rpl31, we speculate that insertion may require both regions. Dissociation might be triggered by a single signaling event given that we observe a concerted release of all four factors during the first hours of embryogenesis (Fig. 1f). Since mammalian Dap1 has been shown to be phosphorylated in an mTOR-dependent manner ${ }^{38}$, this signaling event could be linked to the rise in mTOR signaling after fertilization ${ }^{62}$.

Zygotic ribosomes are first produced after the maternal-to-zygotic transition, an event that, in some species (e.g. in humans), can take place years after the mature oocyte is formed. While ribosome storage is remarkably different in the egg compared to any other so-far described 'dormant' ribosome state in somatic cells, it is intriguing to speculate that similar mechanisms may be used at other stages in the lifetime of animals that require prolonged periods of low metabolic activity, e.g. the diapause in C. elegans (dauer stage). The mechanism we describe here, namely having functionally important sites of the ribosome occupied by evolutionarily conserved factors, two of which are needed for resumption of translation later on, appears ideally suited to accomplish energy-preserving long-term storage of ribosomes in a variety of developmentally programmed and metabolically-induced contexts. 


\section{Methods}

\section{Zebrafish and Xenopus lines and husbandry}

Zebrafish (Danio rerio) were raised according to standard protocols $\left(28^{\circ} \mathrm{C} ; 14 / 10\right.$ hour light/dark cycle). TLAB fish, generated by crossing zebrafish $\mathrm{AB}$ with the natural variant TL (Tupfel Longfin), served as wild-type zebrafish for all experiments. Zebrafish dap, dap $1 b$ and habp 4 mutants were generated as part of this study and are described below. Wild-type Xenopus laevis were obtained from NASCO (USA) and maintained in the IMP animal facility. All fish and Xenopus experiments were conducted according to Austrian and European guidelines for animal research and approved by local Austrian authorities (animal protocols for work with zebrafish: GZ 342445/2016/12 and MA 58-221180-2021-16; animal protocols for work with Xenopus: BMWFW-66.006/0012-WF/II/3b/2014, BMWFW-66.006/0003$\mathrm{WF} / \mathrm{V} / 3 \mathrm{~b} / 2016)$.

\section{Generation of knockout fish lines}

Zebrafish dap, dap 1 b and habp4 mutants were generated by Cas9-mediated mutagenesis ${ }^{63}$. A pool of two guide-RNAs (sgRNAs) targeting the first exon of either dap (chr24:2207121622103555), daplb (chr9:52378482-52386733) or habp4/zgc:103482 (chr21:2039689120402554) were generated according to published protocols ${ }^{63}$ (see Extended Data Table 6 for oligos used for generating sgRNAs). Cas9 protein and sgRNA pools were co-injected into onecell stage TLAB embryos. Putative founders were outcrossed to TLAB, and germline mutations were identified by a size difference in PCR amplicons (see Extended Data Table 6 for primers used for genotyping). Embryos from founders were raised to adulthood. Homozygous knockout fish were generated by crossing heterozygous fish. To generate $\mathrm{dap}^{-/}$, $d a p 1 b^{--}$double mutants, $d a p^{+-}$and $d a p 1 b^{+-}$fish were crossed, and double heterozygous mutants were genotyped and raised to adulthood. Double homozygous mutants (dap ${ }^{-/}$, dap $1 b^{-}$ $\left.{ }^{-}\right)$and wild-type $\left(\right.$dap $^{+/+}$, dap $\left.1 b^{+/+}\right)$siblings were obtained from in-crossing double heterozygous mutants.

\section{Phenotypic characterization}

Adult fish were crossed at least twice prior to phenotypic characterization. Females and males were kept at equal numbers per tank. Pictures of early development were taken at $0.5,1,2,3$, 6 and 24 hours postfertilization (hpf) on a ZEISS Stemi 508 stereo microscope with camera (2x magnification, FlyCapture 2 software). To calculate the fertilization rate, the number of fertilized eggs was divided by the number of total eggs. Clutch size was calculated as the sum of fertilized and unfertilized eggs. Survival rate was calculated by dividing live larvae at 24 hpf by the number of fertilized eggs. The size of the embryos was measured as area $\left(\mathrm{mm}^{2}\right)$ at 6 hpf in Fiji. Statistical analysis was performed in GraphPad Prism using Mann-Whitney test (for two groups, $p$-value $<0.05$ ) or Kruskal-Wallis test (for multiple groups, $p$-value $<0.05$ ).

\section{Polysome gradients}

Wild-type and mutant embryos were dechorionated with pronase $(1 \mathrm{mg} / \mathrm{mL}) .200$ dechorionated embryos per genotype were lysed in $550 \mu \mathrm{L}$ of lysis buffer $(20 \mathrm{mM}$ Tris-Cl pH 7.5, $30 \mathrm{mM} \mathrm{MgCl} 2,100 \mathrm{mM} \mathrm{NaCl}, 0.25 \%$ Igepal-630 ( $/ / v), 100 \mu \mathrm{g} / \mathrm{mL}$ cycloheximide, 0.5 $\mathrm{mM}$ dithiothreitol (DTT), and $1 \mathrm{mg} / \mathrm{mL}$ heparin). Embryo lysates were incubated for $10 \mathrm{~min}$ on ice and centrifuged for $10 \mathrm{~min}$ at $4^{\circ} \mathrm{C}$ with $20,000 \mathrm{xg}$. $200 \mu \mathrm{L}$ of clarified lysates were loaded onto a continuous $10-50 \%(w / v)$ sucrose gradient prepared in TMS buffer $(20 \mathrm{mM}$ Tris-Cl $\mathrm{pH}$ $7.5,5 \mathrm{mM} \mathrm{MgCl} 2,140 \mathrm{mM} \mathrm{NaCl}$ ). Gradients were centrifuged in a SW40 Ti rotor (Beckman) at $4{ }^{\circ} \mathrm{C}$ and $35,000 \mathrm{rpm}$ for $165 \mathrm{~min}$. Polysome gradients were analyzed using a gradient station (BioComp) coupled to a Model Triax ${ }^{\mathrm{TM}}$ Flow Cell detector (FC-2). The precise location of the 
fractions along the UV-tracing was monitored using the Gradient profiler v1.25 (BioComp) software.

\section{RNA isolation}

Total RNA was isolated from 10 homogenized zebrafish embryos per sample using the RNeasy Mini Kit (Qiagen). RNA concentration was measured on a spectrophotometer (DeNovix DS$11 \mathrm{FX}+$ ). Mutant RNA concentration was normalized to wild-type RNA concentration for each experiment.

\section{Analysis of translation efficiency}

To calculate translational efficiencies over the time-course of embryogenesis, published PolyA + RNASeq data ${ }^{24}$ (GSE32898) and ribosome profiling data ${ }^{23}$ (GSE46512) was preprocessed according to standard bioinformatic procedures. For ribosome profiling data (riboseq), 3' adaptor sequences were removed using bbduk from bbmap v38.26, and trimmed reads were mapped to a set of rRNAs and abundant sequences downloaded from the Illumina iGenome using bowtie2. The remaining reads were aligned to the zebrafish reference genome GRCz10 using tophat2. For RNA-seq analysis, adaptor sequences were removed with bbduk from bbmap v38.26, and abundant reads that mapped to abundant sequences using bowtie 2 were removed, similar to ribo-seq reads. Remaining reads were mapped with tophat 2 to the zebrafish transcriptome using the GRCz10 genome assembly and the Ensembl 82 transcriptome release.

For ribo-seq and RNA-seq, FPKM values for the coding sequence (CDS) were calculated for transcripts with an annotated start and stop codon. FPKM values were defined as read counts over CDS / (CDS length * total read count) $* 10^{9}$. Translational efficiencies were obtained by dividing FPKM of ribo-seq by FPKM of RNA-seq. TE values +0.1 were used for plotting.

\section{Sample collection for ribosome isolation}

The evening prior to the zebrafish egg collections, male and female zebrafish were separated in breeding cages. To collect mature, un-activated eggs, female zebrafish were anesthetized in the morning using $0.1 \%(w / v)$ Tricaine $(25 \mathrm{x}$ stock solution in $\mathrm{dH} 2 \mathrm{O}$, buffered to $\mathrm{pH} 7-7.5$ with $1 \mathrm{M}$ Tris $\mathrm{pH}$ 9.0). After being gently dried on a paper towel, the female was transferred to a dry petri dish, and eggs were carefully expelled from the female by applying mild pressure on the fish belly with a finger and stroking from anterior to posterior. The eggs were separated from the female using a small paintbrush, and the female was transferred back to the breeding cage filled with fish water for recovery. To prevent activation, eggs were kept in sorting medium (Leibovitz's medium, $0.5 \% \mathrm{BSA}, \mathrm{pH} 9)^{64}$ at room temperature (RT). In the case of zebrafish embryos (1,000 embryos per sample for mass spectrometry (MS) and electron cryomicroscopy (Cryo-EM), and 5,000 embryos per sample for crosslinking-MS), eggs were collected in Petri dishes with blue water, which consist of fish water, $0.025 \%(v / v)$ Instant Ocean salts (Aquarium Systems, 218035), and $0.0001 \%(v / v)$ methylene blue (Sigma-Aldrich, M9140), $\mathrm{pH} 7$. Embryos were incubated at $28^{\circ} \mathrm{C}$, collected at the desired time points (1 hpf and $6 \mathrm{hpf}$ ), and incubated with $1 \mathrm{mg} / \mathrm{mL}$ of pronase for $5 \mathrm{~min}$ at $\mathrm{RT}$ for dechorionation.

To obtain samples from Xenopus, egg collection and in vitro fertilization were performed following a previously described protoco ${ }^{65}$. In brief, sexually matured wild-type females were primed with $50 \mathrm{IU}$ of pregnant mare serum gonadotropin (PMSG, ProSpec, HOR-272) one week before the experiments. At the evening before egg collection, PMSG-primed females were injected with $500 \mathrm{IU}$ of human chorionic gonadotropin (hCG, Sigma-Aldrich, CG10). Freshly laid eggs were collected and chemically dejellied in 2\% (w/v) L-cysteine/1xMMR (Marc's Modified Ringer) pH 7.8 before ribosome extraction. For the 24 hpf sample, freshly laid eggs were in vitro fertilized with wild-type sperm and dejellied in $2 \%(w / v)$ L- 
cysteine/0.1xMMR pH 7.8 at the 1-cell stage. Embryos were allowed to develop at RT for 24 $\mathrm{h}$ before ribosome extraction.

\section{Ribosome isolation}

Samples were washed in 2 volumes of lysis buffer, containing $20 \mathrm{mM}$ HEPES-KOH pH 7.4, $150 \mathrm{mM} \mathrm{KCl}, 10 \mathrm{mM} \mathrm{MgCl} 2,0.5 \mathrm{mM}$ DTT, rRNasin (Promega), 0.25\% RNaseOUT ( $v / v$ ) (ThermoFisher), $0.25 \%$ SUPERaseIN ( $v / v)$ (ThermoFisher), $0.25 \%$ Igepal $(v / v)$, and cOmpleteTM-EDTA-free protease inhibitor (Roche). Embryos were lysed in $2 \mathrm{~mL}$ (for 1,000 embryos) or $5 \mathrm{~mL}$ (for 5,000 embryos) of lysis buffer using a pre-cooled Dounce homogenizer. $100 \mu \mathrm{g} / \mathrm{mL}$ of cycloheximide was added during lysis. Following incubation on ice for $5 \mathrm{~min}$,

the supernatant was cleared by centrifugation at $20,000 \mathrm{xg}$ for $10 \mathrm{~min}$ at $4^{\circ} \mathrm{C}$. Ribosome isolation was adapted from ${ }^{66}$. Briefly, the clarified supernatant was loaded onto a $30 \%(w / v)$ sucrose cushion prepared in buffer A (20 mM Tris-Cl pH 7.5, $2 \mathrm{mM} \mathrm{Mg}(\mathrm{OAc})_{2}$ and $150 \mathrm{mM}$ $\mathrm{KCl}$ ) and centrifuged at $116,000 \mathrm{xg}$ for $5 \mathrm{~h}$ at $4^{\circ} \mathrm{C}$ in a TL100.3 rotor (Beckman). The ribosome pellet was resuspended by orbital shaking $\left(1 \mathrm{~h}\right.$ at $\left.4{ }^{\circ} \mathrm{C}\right)$ in buffer $\mathrm{B}$, consisting of $20 \mathrm{mM}$ Tris$\mathrm{Cl} \mathrm{pH} \mathrm{7.5,} 6 \mathrm{mM} \mathrm{Mg}(\mathrm{OAc})_{2}, 150 \mathrm{mM} \mathrm{KCl}, 6.8 \%$ sucrose $(w / v), 1 \mathrm{mM}$ DTT, $0.25 \%$ RNasin Plus $(v / v)$ (ThermoFisher), and $0.25 \%$ RNase Inhibitor $(v / v)$, (Promega). Resuspended ribosomes were loaded onto a $15-30 \%(w / v)$ sucrose gradient prepared in buffer C $(100 \mathrm{mM}$ $\mathrm{KCl}, 5 \mathrm{mM} \mathrm{Mg}(\mathrm{OAc})_{2}, 20 \mathrm{mM}$ HEPES-KOH pH 7.6, $1 \mathrm{mM}$ DTT, and $\left.10 \mathrm{mM} \mathrm{NH}_{4} \mathrm{Cl}\right)$ and centrifuged for $16 \mathrm{~h}$ at $4^{\circ} \mathrm{C}$ with 18,000 rpm in an SW60Ti rotor (Beckman). Gradients were hand-fractionated and ribosome concentrations were determined by absorbance at $260 \mathrm{~nm}$ $\left(\mathrm{A}_{260}\right)$ and SDS-PAGE.

To isolate ribosomes from rabbit reticulocyte lysates (RRL) (Green Hectares; composition and preparation of the lysate are described in the section "In vitro translation assays" below), RRL was supplemented with $20 \mu \mathrm{M}$ recombinant zebrafish Dap1b protein and incubated for $1 \mathrm{~h}$ at $30^{\circ} \mathrm{C}$. A one-step centrifugation of $1 \mathrm{~mL}$ of in vitro translation reaction was performed over 3 $\mathrm{mL}$ of a $30 \%(w / v)$ sucrose cushion prepared in RNC buffer $(50 \mathrm{mM}$ HEPES-KOH pH 7.4, $100 \mathrm{mM} \mathrm{KOAc}, 5 \mathrm{mM} \mathrm{Mg}(\mathrm{OAc})_{2}$, and $1 \mathrm{mM}$ DTT) in a TLA100.3 rotor (Beckman) at 116,000 $\mathrm{xg}$ for $5 \mathrm{~h}$. The ribosome pellet was resuspended in $120 \mathrm{~mL}$ of RNC buffer.

For MS and cryo-EM, sucrose was omitted from the gradient fractions by loading the resuspended ribosome solution on a Zeba ${ }^{\mathrm{TM}}$ Spin Desalting Columns (7K MWCO).

\section{Digest of total cell lysates and ribosomes for mass spectrometry (MS)}

Total cell lysates and isolated ribosomes from zebrafish eggs and embryos and from Xenopus eggs were either directly denatured in $8 \mathrm{M}$ urea in $100 \mathrm{mM}$ ammoniumbicarbonate (ABC) buffer or acetone precipitated before being dissolved in $8 \mathrm{M}$ urea in $100 \mathrm{mM} \mathrm{ABC}$. DTT was added to a final concentration of $10 \mathrm{mM}$ and the sample was incubated $1 \mathrm{~h}$ at $37^{\circ} \mathrm{C}$. Alkylation was performed by adding iodoacetamide (IAA) to a final concentration of $20 \mathrm{mM}$ and incubating for $30 \mathrm{~min}$ at RT in the dark. The reaction was quenched by addition of $5 \mathrm{mM}$ DTT and incubated again 30 min at RT.

Samples were diluted to $6 \mathrm{M}$ urea with $100 \mathrm{mM}$ ABC followed by addition of Lys-C (FUJIFILM Wako Pure Chemical Corporation) at a ratio of $1: 100$ and incubation at $37^{\circ} \mathrm{C}$ for $2 \mathrm{~h}$. The samples were diluted to $2 \mathrm{M}$ urea with $100 \mathrm{mM}$ ABC. Trypsin (Promega, Trypsin Gold) was added at a ratio of 1:100 and incubated at $37^{\circ} \mathrm{C}$ overnight. $500 \mathrm{ng}$ of each sample were analyzed by liquid chromatography-mass spectrometry (LC-MS/MS).

\section{Crosslinking of purified ribosomes and digest for mass spectrometry (MS)}

For elution of zebrafish $1 \mathrm{hpf}$ and Xenopus egg ribosome fractions used in crosslinking-MS experiments, buffer $\mathrm{C}$ was adjusted to $20 \mathrm{mM}$ HEPES-KOH pH 7.6, $100 \mathrm{mM} \mathrm{KCl}$, and $5 \mathrm{mM}$ 
$\mathrm{Mg}(\mathrm{OAc})_{2} .0 .5 \mathrm{mM}$ of disuccinimidyl sulfoxide (DSSO) from a $5 \mathrm{mM}$ stock solution (in dimethyl sulfoxide, DMSO) was added to $10 \mu \mathrm{L}$ of $500 \mathrm{nM}$ ribosomes and incubated for 45 $\mathrm{min}$ at RT. The reaction was quenched by adding Tris- $\mathrm{Cl} \mathrm{pH} 7.5$ to a final concentration of 100 $\mathrm{mM}$ and incubated for $15 \mathrm{~min}$ at RT. The band pattern of the crosslinking reaction was analyzed by SDS-PAGE.

DSSO crosslinked ribosomes were denatured in $8 \mathrm{M}$ urea, $100 \mathrm{mM}$ ABC followed by reduction, alkylation and proteolytic digest using Lys-C and trypsin (both added 1:20) as described above. Digests were acidified to 1\% TFA and desalted using Oasis HLB Sorbent (Oasis HLB Microelution plate, Waters) according to the manufacturer's description. Peptides were eluted with $2 \times 30 \mu \mathrm{L} 70 \%$ acetonitrile (ACN), $0.1 \%$ TFA and the sample was concentrated under reduced pressure to $20 \mu \mathrm{L}$. The samples were supplemented with $5 \%$ DMSO. To enrich for crosslinked peptides, the samples were fractionated by size-exclusion chromatography (SEC) on a TSKgel SuperSW2000 column $(300 \mathrm{~mm} \times 4.5 \mathrm{~mm} \times 4 \mu \mathrm{m}$, Tosoh Bioscience), which was operated at $200 \mu \mathrm{L} / \mathrm{min}$ in $30 \%$ ACN, $0.1 \%$ TFA. Fractions were collected every minute, and ACN was removed under reduced pressure. DMSO was again added to $5 \%$.

\section{LC-MS/MS}

Generated peptides were analyzed on a nano-reversed phase HPLC (RSLC nano system, ThermoFisher) coupled to a Q Exactive HF-X mass spectrometer (ThermoFisher), equipped with a Nanospray Flex ion source (ThermoFisher).

Peptides were loaded onto a trap column (PepMap C18, $5 \mathrm{~mm} \times 300 \mu \mathrm{m}$ ID, $5 \mu \mathrm{m}$ particles, $100 \AA$ pore size) at a flow rate of $25 \mu \mathrm{L} / \mathrm{min}$ using $0.1 \%$ TFA as mobile phase. After $10 \mathrm{~min}$, the trap column was switched in line with the analytical column (PepMap C18, $500 \mathrm{~mm} \times 75$ $\mu \mathrm{m} \mathrm{ID,} 2 \mu \mathrm{m}, 100 \AA$, ThermoFisher), which was operated at a flowrate of $230 \mathrm{nl} / \mathrm{min}$ at $30^{\circ} \mathrm{C}$. For separation a solvent gradient was applied, starting with $98 \%$ buffer A $(0.1 \%$ formic acid in water) and $2 \%$ buffer B ( $80 \%$ ACN, $0.1 \%$ formic acid), followed by an increase to $35 \%$ buffer $\mathrm{B}$ over the next 180 or $360 \mathrm{~min}$ for digested lysates and ribosomes. For unfractionated and SEC-enriched crosslinked samples, a gradient to $40 \%$ buffer B in 180 or $120 \mathrm{~min}$, respectively, was used. This was followed by a steep gradient to $90 \%$ buffer B in $5 \mathrm{~min}$, staying there for five min and decreasing to $2 \%$ buffer B in another 5 min.

For the digested lysates and ribosomes, the mass spectrometer was operated in data-dependent mode, using a full scan ( $\mathrm{m} / \mathrm{z}$ range $375-1500$, resolution of 60,000 , target value 1E6) followed by MS/MS scans of the 10 most abundant ions. MS/MS spectra were acquired using a normalized collision energy of 28 , isolation width of $1.4 \mathrm{~m} / \mathrm{z}$, resolution of 30,000 , maximum fill time of $105 \mathrm{~ms}$ and a target value of 1E5. Precursor ions selected for fragmentation (excluding charge state $1,7,8,>8$ ) were put on a dynamic exclusion list for $60 \mathrm{~s}$. Additionally, the minimum AGC target was set to $5 \mathrm{E} 3$ and intensity threshold was calculated to be $4.8 \mathrm{E} 4$. The peptide match feature was set to preferred, and the exclude isotopes feature was enabled.

For crosslinked samples, the mass spectrometer was operated in data-dependent mode, using a full scan (m/z range 350-1600, resolution of 120,000 and a target value of 1E6) followed by MS/MS scans of the 15 most abundant ions. MS/MS spectra were acquired using a stepped normalized collision energy of $27+/-6$, isolation width of $1 \mathrm{~m} / \mathrm{z}$, resolution of 30,000 , maximum fill time of $150 \mathrm{~ms}$ and a target value of 5E4. Precursor ions selected for fragmentation (excluding charge state $1,2,>7$ ) were put on a dynamic exclusion list for $30 \mathrm{~s}$. Additionally, the minimum AGC target was set to $5 \mathrm{E} 3$, and intensity threshold was calculated to be $3.3 \mathrm{E} 4$. The peptide match feature was set to preferred, and the exclude isotopes feature was enabled.

\section{Analysis of MS data}


For peptide identification, the RAW-files were loaded into Proteome Discoverer (v2.5.0.402, ThermoFisher). All hereby created MS/MS spectra were searched using MSAmanda v2.5.0.16129, Engine v2.0.0.1612967. For the first step search, the RAW-files were searched against a combined zebrafish sequence database (Danio_rerio GRCz11) and Uniprot downloaded on $21^{\text {st }}$ March 2019 (58,524 sequences; 34,079,443 residues), and against the Xenopus laevis Uniprot proteome UP000186698. All RAW-files were supplemented with common contaminants using the following search parameters: (i) the peptide mass tolerance was set to $\pm 5 \mathrm{ppm}$ and the fragment mass tolerance to $\pm 8 \mathrm{ppm}$, and (ii) the maximal number of missed cleavages was set to 2 using tryptic enzymatic specificity. The result was filtered to $1 \%$ FDR on protein level using Percolator algorithm ${ }^{68}$ integrated in Proteome Discoverer. A subdatabase was generated for further processing. For the second step, the RAW-files were searched against the created sub-database. Oxidation on methionine, deamidation on asparagine and glutamine, phosphorylation on serine, threonine and tyrosine; iodoacetamide derivative on cysteine, beta-methylthiolation on cysteine and carbamylation on lysine were set as variable modifications. Monoisotopic masses were searched within unrestricted protein masses for tryptic enzymatic specificity. The peptide mass tolerance was set to $\pm 5 \mathrm{ppm}$ and the fragment mass tolerance to $\pm 8 \mathrm{ppm}$. The maximal number of missed cleavages was set to 2 . The result was filtered to $1 \%$ FDR on peptide level using Percolator algorithm integrated in Proteome Discoverer. The localization of the post-translational modification sites within the peptides was performed with the tool ptmRS, based on the tool phosphoRS ${ }^{69}$. Peptide areas have been quantified using in-house-developed tool apQuant ${ }^{70}$. Proteins were quantified by summing unique and razor peptides and applying the intensity-based absolute quantification (iBAQ) calculation. Normalization of protein abundances was performed on the sum of areas of all identified ribosomal proteins. Statistical significance of differentially expressed proteins was determined using limma ${ }^{71}$.

\section{Analysis of crosslinking-MS data}

Data analysis was performed within Proteome Discoverer v2.5.0.402 using MS Annika $(\mathrm{v} 1.0 .18345)^{72}$. The workflow tree consisted of the MS Annika Detector node (MS tolerance 10 ppm, crosslink modification: DSSO $+185.004 \mathrm{Da}$ at lysine and at protein N-termini, diagnostic ions: $138.0911 ; 155.179 ; 170.0634 ; 187.0900$, crosslink modification addition: 18.010565, doublet pair selection in combined mode) followed by MS Annika Search (full tryptic digest, $5 / 10 \mathrm{ppm}$ peptide/fragment mass tolerance, maximal 3 missed cleavages, carbamidomethyl +57.021 Da at cysteine as static and oxidation $+15.995 \mathrm{Da}$ at methionine as dynamic modification) and completed with MS Annika Validator (1\% FDR cutoff at CSM and crosslink level, separate Intra/Inter-link FDR set to false). The search was performed against a database generated from a MS analysis of a digested non-crosslinked zebrafish (containing 1,092 proteins) or Xenopus (containing 1,173 proteins) ribosome sample, respectively. To generate these specific databases the two non-crosslinked samples were searched using MS Amanda against the zebrafish Uniprot reference proteome UP000000437 (downloaded 202009-15, 46,847 sequences, 24,556,292 residues) or Xenopus laevis Uniprot proteome UP000186698 (downloaded 2020-01-07, 43,235 sequences, 19,251,456 residues), both supplemented with common contaminants. Search parameters were set as described for time course experiments but using 5/10 ppm peptide/fragment mass tolerance and maximum 3 missed cleavages. Carbamidomethylation on cysteine was set as a fixed modification, oxidation on methionine, deamidation on asparagine and glutamine and acetylation on the protein $\mathrm{N}$-terminus were set as variable modifications.

To validate the quality of the chosen search strategy, an additional crosslink search was performed using equal settings but a database file containing sequences aligned to the dormant ribosome structures of Xenopus and zebrafish. The resulting crosslinks at 1\% FDR were plotted 
onto the structure using ChimeraX v1.1 ${ }^{73}$, and the distance between connected amino acids $(\mathrm{C} \alpha-\mathrm{C} \alpha$ distance) was measured. Crosslinks including $\mathrm{C} \alpha$ residues missing in the structure file were removed, yielding 360 and 178 crosslinks aligned to the zebrafish and Xenopus structures, respectively.

\section{DNA constructs and mRNA synthesis}

dap, dap 1b, habp4, renilla luciferase and zebrafish $\beta$-globin constructs for in vitro translation in rabbit reticulocyte lysate (RRL) (Green Hectares) were cloned into pCDNA3.1 by Gibson assembly ${ }^{74}$. Zebrafish coding sequences were amplified from cDNA. Dap, dapl1 and dap1 coding sequences from other species were ordered as Gblocks from IDT (amino acid sequences are shown in Extended Data Fig. 5). Capped mRNAs of all cloned constructs were synthesized with the Sp6 mMessage Machine kit (Ambion) according to the manufacturer's protocol, using linearized plasmids as templates.

\section{Recombinant protein expression and purifications}

Zebrafish dap $1 b$ and dap coding sequences were codon optimized for E. coli, cloned into a vector providing an $\mathrm{N}$-terminal $6 \mathrm{xHis}$ tag followed by a $3 \mathrm{C}$ protease-cleavage site, and transformed into BL21(DE3) E. coli cells. For Dap1b expression, cells were grown in LB at $37^{\circ} \mathrm{C}$ until reaching an $\mathrm{OD}_{600}$ of 0.7 , induced with $0.5 \mathrm{mM}$ IPTG, and grown overnight at $18^{\circ} \mathrm{C}$. For Dap expression, bacterial cultures were grown on terrific broth medium supplemented with $1.5 \%(w / v)$ lactose for $1 \mathrm{~h}$ at $37^{\circ} \mathrm{C}$, and for $23 \mathrm{~h}$ at $18^{\circ} \mathrm{C}$. Cells were pelleted, resuspended in lysis buffer $(50 \mathrm{mM}$ Tris-Cl pH 8.0, $300 \mathrm{mM} \mathrm{NaCl}$, cOmplete Protease Inhibitor Cocktail [Merck]) and lysed by sonication (19 mm probe, 4 min: pulse on $1 \mathrm{~s}$, pulse off $2 \mathrm{~s}, 60 \%$ amplitude). The intracellular soluble fraction was isolated by centrifugation at $20,000 \mathrm{xg}, 30$ min, $4^{\circ} \mathrm{C}$. Imidazole was added to a final concentration of $20 \mathrm{mM}$. The lysate was loaded onto a $5 \mathrm{~mL}$ HisTrap FF column (Merck), washed with buffer A (50 mM Tris-Cl pH 8.0, $300 \mathrm{mM}$ $\mathrm{NaCl}$ ), and eluted with buffer A supplemented with $500 \mathrm{mM}$ imidazole. $1 \mathrm{mg}$ of $3 \mathrm{C}$ protease was added to the elution fraction during dialysis overnight against buffer A. The cleaved protein was loaded onto a $5 \mathrm{~mL}$ HisTrap FF column (Merck); the flow-through, containing the cleaved protein, was concentrated (Vivaspin 20, MWCO $5 \mathrm{kDa}$ ) and loaded onto a Superdex 75 26/60 column (GE Life Sciences) equilibrated with SEC buffer (20 mM HEPES-KOH pH $7.5,150 \mathrm{mM} \mathrm{KCl})$.

\section{In vitro translation (IVT) assays}

Rabbit reticulocyte lysate (RRL; Green Hectares) was optimized for translation as previously described $^{75,76}$. The final reaction contained 35\% $(v / v)$ RRL, $20 \mathrm{mM}$ HEPES-KOH pH 7.4, $10 \mathrm{mM}$ KOH, $50 \mathrm{mM}$ KOAc, $1 \mathrm{mM}$ ATP, $1 \mathrm{mM}$ GTP, $12 \mathrm{mM}$ creatine phosphate, $0.1 \mathrm{mg} / \mathrm{mL}$ tRNAs (Merck), $40 \mu \mathrm{g} / \mathrm{mL}$ creatine kinase, $2 \mathrm{mM} \mathrm{MgCl} 2,1 \mathrm{mM}$ reduced glutathione, $0.3 \mathrm{mM}$ spermidine, and $40 \mu \mathrm{M}$ of each of the 20 amino acids. $1 \mu \mathrm{g}$ of mRNA was added to a total volume of $30 \mu \mathrm{L}$ of RRL. To compare translation of different factors, equimolar amounts of mRNA were added per reaction. Reactions were performed at $30^{\circ} \mathrm{C}$ for $60 \mathrm{~min}$ (unless stated otherwise). To assess ribosome binding, $250 \mu \mathrm{L}$ of the reaction was loaded onto a $30 \%(w / v)$ sucrose cushion in ribosome-stabilizing RNC buffer (50 mM HEPES-KOH pH 7.4, $100 \mathrm{mM}$ $\mathrm{KOAc}, 5 \mathrm{mM} \mathrm{Mg}(\mathrm{OAc})_{2}$, and $\left.1 \mathrm{mM} \mathrm{DTT}\right)$, and centrifuged for $5 \mathrm{~h}$ with $116,000 \mathrm{xg}$ at $4^{\circ} \mathrm{C}$ in a TLA100.3 rotor (Beckman). Binding to the different fractions was assessed by Western blot. To test for translation inhibition of recombinant Dap and Dap1b proteins, $0.1,1,2,5,10$ or 50 $\mu \mathrm{M}$ recombinant protein in protein buffer $(20 \mathrm{mM}$ HEPES-KOH pH 7.4, $150 \mathrm{mM} \mathrm{KCl})$ and 1 $\mu \mathrm{g}$ Renilla mRNA were added to RRL to a total volume of $25 \mu \mathrm{L}$. Bac7(1-35) synthetic peptide (1-RRIRPRPPRLPRPRPRPLPFPRPGPRPIPRPLPFP-35) was used as a positive control. BSA served as negative control. Reactions were performed at $30^{\circ} \mathrm{C}$ for $90 \mathrm{~min}$. Renilla luciferase 
activity (Promega, E1960) was measured in duplicates on a Synergy H1 plate reader (BioTek). Samples were normalized to buffer control containing $1 \mu \mathrm{g}$ of renilla luciferase mRNA. Relative IC50 was calculated in GraphPad Prism using nonlinear regression and the equation [Inhibitor] vs. response - Variable slope (four parameters).

\section{Western blotting}

Western blotting was performed following standard protocols. In brief, protein samples were boiled for $5 \mathrm{~min}$ in 1x Laemmli sample buffer and separated on an SDS-PAGE using MiniPROTEAN ${ }^{\circledR}$ TGXTM Precast Protein Gels or 10-20\% Mini-PROTEAN ${ }^{\circ}$ Tris-Tricine Gels (Bio-Rad). Blotting was performed on a nitrocellulose membrane (GE Healthcare) using a wetblot system (BioRad). The following antibodies were used: anti-FLAG (mouse, 1:1000, SigmaAldrich F1804), anti-eEF2 (rabbit, 1:1000, Proteintech, 20107-1-AP) and anti-RPL3 (rabbit, 1:1000, GeneTex, GTX124464). The chemiluminescent signal was quantified using ImageJ 1.8.0_172. To quantify ribosome binding of FLAG-tagged proteins after in vitro translation, signal intensities were simultaneously measured for FLAG, RPL3 and eEF2 for all three membranes (total reaction, supernatant, ribosome pellet). The ratio between the FLAG signal in the supernatant and the ribosome pellet was normalized to the total amount translated (FLAG signal in the total reaction) and to sample loading (RPL3 and eEF2 signals).

\section{Grid preparation for cryo-EM}

A $2 \mathrm{~nm}$ thick continuous carbon film was produced on an Auto306 high vacuum evaporator (Boc Edwards). This film was floated onto Quantifoil $\mathrm{Cu}$ 3.5/1, 200 mesh grids, and grids were dried. Grids were glow-discharged for $1 \mathrm{~min}$ in a SCD005 sputter coater (Bal-Tec) at $20 \mathrm{~mA}$. $4 \mu \mathrm{l}$ of sample (at a concentration of $200 \mathrm{ng} / \mu \mathrm{l}$ of RNA, which corresponds to $\sim 100 \mathrm{nM}$ ribosomes) were applied to the grid and incubated for $60 \mathrm{~s}$ at $70 \%$ humidity and $4{ }^{\circ} \mathrm{C}$ in a Leica EM GP. Subsequently, grids were blotted for $2 \mathrm{~s}$ using the proximity sensor and plunge-frozen in liquid ethane at $-180^{\circ} \mathrm{C}$.

\section{Cryo-EM data collection}

All grids were screened on a Glacios TEM (ThermoFisher) to check for particle distribution and grid quality. Grids that past the evaluation were recorded on a Titan Krios microscope equipped either with a Falcon $3 \mathrm{EC}$ or a K3 detector. A pixel size of about $1 \AA / \mathrm{px}$ was chosen, and micrographs with a total dose of about 40 e/ $\AA 2$ fractionated in 39 frames were collected with a target defocus between -1 and $-2.5 \mu \mathrm{m}$.

\section{Electron microscopy data processing}

Most data processing was performed in Cryosparc version 2.16.0 ${ }^{77}$. Micrographs were motion corrected and dose weighted using Patch motion correction. CTF (Contrast Transfer Function) parameters were determined using Patch CTF. About 500 particles were manually selected and 2D classified to create templates. Automated particle picking was performed using these templates. Picking was manually inspected, and a threshold that excludes low signal-to-noise and high signal-to-dirt false positive picks and minimizes the false negative picks was chosen. The remaining particles were extracted with a box size between 440 and 512 and subjected to 2D classification. Classes that showed clear ribosomal densities were selected and subjected to heterogeneous ab-initio model generation. Subsequently, the remaining particles were 3D refined to generate a consensus model. Afterwards, optics groups were assigned to the individual particles depending on the applied beam shift during recording. Global and local CTF refinements were then performed, and all particles were refined using non-uniform refinement. To generate masks for local refinements, the density was filtered to $20 \AA$, and subdensities of the entire 40S subunit and the head region were generated using the Volume eraser tool in UCSF Chimera 1.13.1 ${ }^{78}$. These densities were binarized, extended by 7 pixels and 
another 7 pixels as soft edge. Locally refined maps using the corresponding masks were obtained in Cryosparc, and composite maps were generated with the vop maximum command in UCSF Chimera 1.13.1 ${ }^{78}$.

To analyze particle dynamics and heterogeneity, the consensus refined particles were subjected to CryoDRGN analysis ${ }^{44}$. Due to the large pixel box size of our data sets, the dimensions of the ribosome particles were reduced to allow for feasible computation. The particles were down-sampled to a box size of 128 or 256 , and two iterative rounds of the CryoDRGN VAE were trained using a network with 3 decoder and 3 encoder layers with 1024 as outer dimensions and a bottleneck latent dimension of 8 . The resulting $\mathrm{z}$ values were classified using kmeans clustering and gaussian mixture models and plotted using a UMAP approach.

\section{Model building and refinement}

The molecular models of the $1 \mathrm{hpf}$ and $6 \mathrm{hpf}$ ribosomes from zebrafish were built using PDB$4 \mathrm{UG} 0^{79}$ as an initial model for core ribosomal proteins and rRNA. In the case of the $1 \mathrm{hpf}$ zebrafish ribosome, additional factors were modeled from previously published structures: eIF5A from PDB-5DAT, and eEF2 and Habp4 (SERBP1 paralog) from PDB-6MTE ${ }^{31}$. All chains were first rigid body-fitted in Coot $0.8 .9^{80,81}$ and manually mutated to the zebrafish sequences, taking MS data from purified zebrafish ribosomes (protein identity and abundance) into consideration. In the case of Habp4, another protein (Serbp1a, Uniprot ID F1Q5Q3) could also fit into the 1 hpf ribosome density. Given that Habp4 was more abundant than Serbpla in $1 \mathrm{hpf}$ zebrafish ribosomes according to MS, Habp4 was modeled.

The dormant ribosome structure from Xenopus eggs was built based on the 60S subunit of the $1 \mathrm{hpf}$ zebrafish ribosome, and the 40S subunit of the $6 \mathrm{hpf}$ zebrafish ribosome, given that both 40S subunits showed the same swiveled conformation. Protein and rRNA molecules from zebrafish were mutated to the corresponding sequences in Xenopus, taking into consideration the MS data from purified Xenopus ribosomes. Dapl1.S was built de novo in Coot and then used as a template to build Dap1b in the zebrafish $1 \mathrm{hpf}$ ribosome. Although two Dap proteins (Dapl1.S and Dapl1.L in Xenopus, and Dap1b and Dap in zebrafish) could fit into the corresponding densities, Dapl1.S and Dap1b were modeled according to gene expression data (Extended Data Fig. 6) and protein abundance (measured by MS).

The rabbit ribosome model with recombinant zebrafish Dap1b was built using $6 \mathrm{MTE}^{31}$ as an initial model for core ribosomal proteins, rRNA, eEF2 and SERBP1. eIF5A was modeled using PDB-5DAT as reference. All models were manually adjusted to fit the observed density in Coot $0.8 .9^{80,81}$ and real-space-refined using Phenix 1.17.1 ${ }^{82}$. The 40S subunit of the rabbit ribosome was only submitted to rigid body fitting in Phenix. Working files were saved in PDB and the final models were converted to mmCIF using PDB extract ${ }^{83}$. Cryo-EM densities and models were visualized using UCSF ChimeraX version $0.9 \overline{1^{73}}$.

\section{Data availability}

Cryo-EM maps and molecular models have been deposited in the Protein Data Bank (PDB) with IDs 7OYA (zebrafish $1 \mathrm{hpf}$ ), 7OYB (zebrafish $6 \mathrm{hpf}$ ), 7OYC (Xenopus eggs) and 7OYD (rabbit ribosome with zebrafish Dap1b), and in the Electron Microscopy Data Bank with accession codes EMD-13111 (zebrafish 1 hpf), EMD-13112 (zebrafish 6 hpf), EMD-13113 (Xenopus egg ribosome) and EMD-13114 (rabbit ribosome with zebrafish Dap1b). Raw micrographs, particle stacks and CryoDRGN models were deposited at the EMPIAR Database. Mass spectrometry proteomics data have been deposited to the ProteomeXchange Consortium via the PRIDE ${ }^{84}$ partner repository with the dataset identifier PXD026866. 


\section{References}

1. Burkholder, G. D., Comings, D. E. \& Okada, T. A. A storage form of ribosomes in mouse oocytes. Experimental Cell Research 69, 361-371 (1971).

2. Alberts, B. et al. Eggs. Molecular Biology of the Cell. 4th edition (2002).

3. Locati, M. D. et al. Expression of distinct maternal and somatic 5.8S, 18S, and 28S rRNA types during zebrafish development. RNA 23, 1188-1199 (2017).

4. Locati, M. D. et al. Linking maternal and somatic 5S rRNA types with different sequencespecific non-LTR retrotransposons. RNA 23, 446-456 (2017).

5. Cenik, E. S. et al. Maternal Ribosomes Are Sufficient for Tissue Diversification during Embryonic Development in C. elegans. Developmental Cell 48, 811-826.e6 (2019).

6. Wegnez, M. \& Monier, R. Sequence heterogeneity of 5 S RNA in Xenopus laevis. FEBS Letters 25, 13-20 (1972).

7. Woodland, H. R. Changes in the polysome content of developing Xenopus laevis embryos. Developmental Biology 40, 90-101 (1974).

8. Brandis, J. W. \& Raff, R. A. Translation of oogenetic mRNA in sea urchin eggs and early embryos. Demonstration of a change in translational efficiency following fertilization. Dev Biol 67, 99-113 (1978).

9. Danilchik, M. V. \& Hille, M. B. Sea urchin egg and embryo ribosomes: differences in translational activity in a cell-free system. Dev Biol 84, 291-298 (1981).

10. Kronja, I. et al. Widespread Changes in the Posttranscriptional Landscape at the Drosophila Oocyte-to-Embryo Transition. Cell Reports 7, 1495-1508 (2014).

11. Chassé, H., Boulben, S., Cormier, P. \& Morales, J. Translational Control of Canonical and Non-Canonical Translation Initiation Factors at the Sea Urchin Egg to Embryo Transition. Int J Mol Sci 20, (2019).

12. Bachvarova, R. \& De Leon, V. Stored and polysomal ribosomes of mouse ova. Developmental Biology 58, 248-254 (1977).

13. Subtelny, A. O., Eichhorn, S. W., Chen, G. R., Sive, H. \& Bartel, D. P. Poly(A)-tail profiling reveals an embryonic switch in translational control. Nature 508, 66-71 (2014).

14. Stebbins-Boaz, B., Cao, Q., Moor, C. H. de, Mendez, R. \& Richter, J. D. Maskin Is a CPEB-Associated Factor that Transiently Interacts with eIF-4E. Molecular Cell 4, 10171027 (1999).

15. Cho, P. F. et al. A New Paradigm for Translational Control: Inhibition via 5'-3' mRNA Tethering by Bicoid and the eIF4E Cognate 4EHP. Cell 121, 411-423 (2005).

16. Seefeldt, A. C. et al. Structure of the mammalian antimicrobial peptide Bac7(1-16) bound within the exit tunnel of a bacterial ribosome. Nucleic Acids Res 44, 2429-2438 (2016).

17. Thoms, M. et al. Structural basis for translational shutdown and immune evasion by the Nsp1 protein of SARS-CoV-2. Science (2020) doi:10.1126/science.abc8665.

18. Ben-Shem, A. et al. The Structure of the Eukaryotic Ribosome at 3.0 A Resolution. Science 334, 1524-1529 (2011).

19. Metafora, S., Felicetti, L. \& Gambino, R. The Mechanism of Protein Synthesis Activation After Fertilization of Sea Urchin Eggs. PNAS 68, 600-604 (1971).

20. Gambino, R., Metafora, S., Felicetti, L. \& Raisman, J. Properties of the ribosomal salt wash from unfertilized and fertilized sea urchin eggs and its effect on natural mRNA translation. Biochimica et Biophysica Acta (BBA) - Nucleic Acids and Protein Synthesis 312, 377-391 (1973).

21. Hille, M. B. Inhibitor of protein synthesis isolated from ribosomes of unfertilised eggs and embryos of sea urchins. Nature 249, 556-558 (1974).

22. Chassé, H., Boulben, S., Costache, V., Cormier, P. \& Morales, J. Analysis of translation using polysome profiling. Nucleic Acids Research 45, e15-e15 (2017). 
23. Chew, G.-L. et al. Ribosome profiling reveals resemblance between long non-coding RNAs and 5' leaders of coding RNAs. Development 140, 2828-2834 (2013).

24. Pauli, A. et al. Systematic identification of long noncoding RNAs expressed during zebrafish embryogenesis. Genome Res 22, 577-591 (2012).

25. Wormington, W. M. \& Brown, D. D. Onset of 5 S RNA gene regulation during Xenopus embryogenesis. Developmental Biology 99, 248-257 (1983).

26. Gutierrez, E. et al. eIF5A Promotes Translation of Polyproline Motifs. Molecular Cell 51, 35-45 (2013).

27. Schuller, A. P., Wu, C. C.-C., Dever, T. E., Buskirk, A. R. \& Green, R. eIF5A Functions Globally in Translation Elongation and Termination. Mol Cell 66, 194-205.e5 (2017).

28. Schmidt, C. et al. Structure of the hypusinylated eukaryotic translation factor eIF-5A bound to the ribosome. Nucleic Acids Research 44, 1944-1951 (2016).

29. Rodnina, M. V., Savelsbergh, A., Katunin, V. I. \& Wintermeyer, W. Hydrolysis of GTP by elongation factor G drives tRNA movement on the ribosome. Nature 385, 37-41 (1997).

30. Flis, J. et al. tRNA Translocation by the Eukaryotic 80 S Ribosome and the Impact of GTP Hydrolysis. Cell Reports 25, 2676-2688.e7 (2018).

31. Brown, A., Baird, M. R., Yip, M. C., Murray, J. \& Shao, S. Structures of translationally inactive mammalian ribosomes. eLife 7, e40486 (2018).

32. Hayashi, H. et al. Tight interaction of eEF2 in the presence of Stm1 on ribosome. J Biochem 163, 177-185 (2018).

33. Anger, A. M. et al. Structures of the human and Drosophila 80 S ribosome. Nature 497, 80-85 (2013).

34. Sun, L., Ryan, D. G., Zhou, M., Sun, T.-T. \& Lavker, R. M. EEDA: a protein associated with an early stage of stratified epithelial differentiation. J Cell Physiol 206, 103-111 (2006).

35. Ma, X. et al. Regulation of cell proliferation in the retinal pigment epithelium: Differential regulation of the death-associated protein like-1 DAPL1 by alternative MITF splice forms. Pigment Cell \& Melanoma Research 31, 411-422 (2018).

36. Ma, X. et al. DAPL1, a susceptibility locus for age-related macular degeneration, acts as a novel suppressor of cell proliferation in the retinal pigment epithelium. Hum Mol Genet 26, 1612-1621 (2017).

37. Deiss, L. P., Feinstein, E., Berissi, H., Cohen, O. \& Kimchi, A. Identification of a novel serine/threonine kinase and a novel $15-\mathrm{kD}$ protein as potential mediators of the gamma interferon-induced cell death. Genes Dev. 9, 15-30 (1995).

38. Koren, I., Reem, E. \& Kimchi, A. DAP1, a Novel Substrate of mTOR, Negatively Regulates Autophagy. Current Biology 20, 1093-1098 (2010).

39. Chandrasekaran, V. et al. Mechanism of ribosome stalling during translation of a poly(A) tail. Nat Struct Mol Biol 26, 1132-1140 (2019).

40. Saini, P., Eyler, D. E., Green, R. \& Dever, T. E. Hypusine-containing protein eIF5A promotes translation elongation. Nature 459, 118-121 (2009).

41. Park, M. H., Nishimura, K., Zanelli, C. F. \& Valentini, S. R. Functional significance of eIF5A and its hypusine modification in eukaryotes. Amino Acids 38, 491-500 (2010).

42. Juszkiewicz, S. et al. ZNF598 Is a Quality Control Sensor of Collided Ribosomes. Molecular Cell 72, 469-481.e7 (2018).

43. Li, W. et al. Structural basis for selective stalling of human ribosome nascent chain complexes by a drug-like molecule. Nat Struct Mol Biol 26, 501-509 (2019).

44. Zhong, E. D., Bepler, T., Berger, B. \& Davis, J. H. CryoDRGN: reconstruction of heterogeneous cryo-EM structures using neural networks. Nat Methods 18, 176-185 (2021). 
45. Rossi, D. et al. Evidence for a Negative Cooperativity between eIF5A and eEF2 on Binding to the Ribosome. PLoS One 11, e0154205 (2016).

46. Kao, A. et al. Development of a Novel Cross-linking Strategy for Fast and Accurate Identification of Cross-linked Peptides of Protein Complexes. Mol Cell Proteomics 10, M110.002212 (2011).

47. Van Dyke, N., Baby, J. \& Van Dyke, M. W. Stmlp, a Ribosome-associated Protein, is Important for Protein Synthesis in Saccharomyces cerevisiae under Nutritional Stress Conditions. Journal of Molecular Biology 358, 1023-1031 (2006).

48. Balagopal, V. \& Parker, R. Stm 1 modulates translation after $80 \mathrm{~S}$ formation in Saccharomyces cerevisiae. $R N A$ 17, 835-842 (2011).

49. Wells, J. N. et al. Structure and function of yeast Lso2 and human CCDC124 bound to hibernating ribosomes. PLOS Biology 18, e3000780 (2020).

50. Blobel, G. \& Potter, V. R. Studies on free and membrane-bound ribosomes in rat liver: I. Distribution as related to total cellular RNA. Journal of Molecular Biology 26, 279-292 (1967).

51. Dave, P., Griesbach, E., Roth, G., Mateju, D. \& Chao, J. A. Single-molecule imaging reveals the coupling of translation and mRNA decay. bioRxiv 2021.06.07.447377 (2021) doi:10.1101/2021.06.07.447377.

52. Vecchi, G. et al. Proteome-wide observation of the phenomenon of life on the edge of solubility. PNAS 117, 1015-1020 (2020).

53. Marygold, S. J. et al. The ribosomal protein genes and Minute loci of Drosophila melanogaster. Genome Biol 8, R216 (2007).

54. Fortier, S., MacRae, T., Bilodeau, M., Sargeant, T. \& Sauvageau, G. Haploinsufficiency screen highlights two distinct groups of ribosomal protein genes essential for embryonic stem cell fate. PNAS 112, 2127-2132 (2015).

55. Amsterdam, A. et al. Many ribosomal protein genes are cancer genes in zebrafish. PLoS Biol 2, E139 (2004).

56. Liu, Y. et al. Autophagy-dependent ribosomal RNA degradation is essential for maintaining nucleotide homeostasis during C. elegans development. eLife 7, e36588 (2018).

57. Florin, T. et al. An antimicrobial peptide that inhibits translation by trapping release factors on the ribosome. Nat Struct Mol Biol 24, 752-757 (2017).

58. Greber, B. J., Boehringer, D., Montellese, C. \& Ban, N. Cryo-EM structures of Arx 1 and maturation factors Reil and Jjj1 bound to the 60S ribosomal subunit. Nat Struct Mol Biol 19, 1228-1233 (2012).

59. Ma, C. et al. Structural snapshot of cytoplasmic pre-60S ribosomal particles bound by Nmd3, Lsg1, Tif6 and Reh1. Nat Struct Mol Biol 24, 214-220 (2017).

60. Kargas, V. et al. Mechanism of completion of peptidyltransferase centre assembly in eukaryotes. eLife 8, e44904 (2019).

61. Klingauf-Nerurkar, P. et al. The GTPase Nog1 co-ordinates the assembly, maturation and quality control of distant ribosomal functional centers. eLife 9, e52474 (2020).

62. Chassé, H. et al. Translatome analysis at the egg-to-embryo transition in sea urchin. Nucleic Acids Res 46, 4607-4621 (2018).

63. Gagnon, J. A. et al. Efficient Mutagenesis by Cas9 Protein-Mediated Oligonucleotide Insertion and Large-Scale Assessment of Single-Guide RNAs. PLOS ONE 9, e98186 (2014).

64. Nair, S., Lindeman, R. E. \& Pelegri, F. In vitro oocyte culture-based manipulation of zebrafish maternal genes. Dev Dyn 242, 44-52 (2013).

65. Sive, H. L., Grainger, R. M. \& Harland, R. M. Early Development of Xenopus laevis. (Cold Spring Harbor: Cold Spring Harbor Laboratory Press, 2000). 
66. Khatter, H. et al. Purification, characterization and crystallization of the human $80 \mathrm{~S}$ ribosome. Nucleic Acids Res 42, e49 (2014).

67. Dorfer, V. et al. MS Amanda, a Universal Identification Algorithm Optimized for High Accuracy Tandem Mass Spectra. J. Proteome Res. 13, 3679-3684 (2014).

68. Käll, L., Canterbury, J. D., Weston, J., Noble, W. S. \& MacCoss, M. J. Semi-supervised learning for peptide identification from shotgun proteomics datasets. Nat Methods 4, 923 925 (2007).

69. Taus, T. et al. Universal and confident phosphorylation site localization using phosphoRS. J Proteome Res 10, 5354-5362 (2011).

70. Doblmann, J. et al. apQuant: Accurate Label-Free Quantification by Quality Filtering. $J$ Proteome Res 18, 535-541 (2019).

71. Smyth, G. K. limma: Linear Models for Microarray Data. in Bioinformatics and Computational Biology Solutions Using $R$ and Bioconductor (eds. Gentleman, R., Carey, V. J., Huber, W., Irizarry, R. A. \& Dudoit, S.) 397-420 (Springer, 2005). doi:10.1007/0387-29362-0_23.

72. Pirklbauer, G. J. et al. MS Annika: A New Cross-Linking Search Engine. J. Proteome Res. 20, 2560-2569 (2021).

73. Goddard, T. D. et al. UCSF ChimeraX: Meeting modern challenges in visualization and analysis. Protein Sci 27, 14-25 (2018).

74. Gibson, D. G. et al. Enzymatic assembly of DNA molecules up to several hundred kilobases. Nat Methods 6, 343-345 (2009).

75. Sharma, A., Mariappan, M., Appathurai, S. \& Hegde, R. S. In vitro dissection of protein translocation into the mammalian endoplasmic reticulum. Methods Mol Biol 619, 339-363 (2010).

76. Feng, Q. \& Shao, S. In vitro reconstitution of translational arrest pathways. Methods 137, 20-36 (2018).

77. Punjani, A., Rubinstein, J. L., Fleet, D. J. \& Brubaker, M. A. cryoSPARC: algorithms for rapid unsupervised cryo-EM structure determination. Nat Methods 14, 290-296 (2017).

78. Pettersen, E. F. et al. UCSF Chimera--a visualization system for exploratory research and analysis. J Comput Chem 25, 1605-1612 (2004).

79. Khatter, H., Myasnikov, A. G., Natchiar, S. K. \& Klaholz, B. P. Structure of the human 80S ribosome. Nature 520, 640-645 (2015).

80. Emsley, P. \& Cowtan, K. Coot: model-building tools for molecular graphics. Acta Crystallogr D Biol Crystallogr 60, 2126-2132 (2004).

81. Emsley, P., Lohkamp, B., Scott, W. G. \& Cowtan, K. Features and development of Coot. Acta Crystallogr D Biol Crystallogr 66, 486-501 (2010).

82. Liebschner, D. et al. Macromolecular structure determination using X-rays, neutrons and electrons: recent developments in Phenix. Acta Crystallogr D Struct Biol 75, 861-877 (2019).

83. Yang, H. et al. Automated and accurate deposition of structures solved by X-ray diffraction to the Protein Data Bank. Acta Crystallogr D Biol Crystallogr 60, 1833-1839 (2004).

84. Perez-Riverol, Y. et al. The PRIDE database and related tools and resources in 2019: improving support for quantification data. Nucleic Acids Res 47, D442-D450 (2019).

85. Cabrera-Quio, L. E., Schleiffer, A., Mechtler, K. \& Pauli, A. Zebrafish Ski7 tunes RNA levels during the oocyte-to-embryo transition. PLOS Genetics 17, e1009390 (2021).

86. Session, A. M. et al. Genome evolution in the allotetraploid frog Xenopus laevis. Nature 538, 336-343 (2016). 
Acknowledgements. We thank L.E. Cabrera-Quio for ribosome profiling data analysis; K.R. Gert for providing non-activated zebrafish eggs; the Mass Spectrometry Facility at the Vienna BioCenter Core Facilities GmbH (VBCF), in particular S. Opravil for the processing of our samples, and R. Imre and G. Dürnberger for their help with data analysis; the VBCF Protein Technologies facility, in particular A. Lehner, for the expression and purification of recombinant Dap1b and Dap proteins; M. Madalinski for synthesizing the Bac7 peptide; the VBCF Electron Microscopy Facility for the support and maintenance of the facilities; H. Stark (MPI BPC) for providing additional Krios measurement time; the IMP animal facility personnel, in particular J. König and F. Ecker, for their excellent care of fish and Xenopus; M. Binner, J. Kiraly and A. Bandura for their help with genotyping; E. Tanaka and T. Clausen for funding T.-Y.L. and A.M., respectively; the entire Pauli group for valuable discussions on the project; the VBC RNA Salon and the RNA Deco-SFB community for providing useful feedback and suggestions; A. Andersen (Life Science Editors), C. Plaschka, A. Stark, J. Brennecke, A. Carter, F. Peske, U. Hohmann, E. Calo, A. Shah and A. Blaha for critical reading and valuable feedback on the manuscript. This work was supported by the Institute of Molecular Pathology (IMP), which receives institutional funding from Boehringer Ingelheim and the Austrian Research Promotion Agency (Headquarter grant FFG-852936), and funding from the FWF START program (Y 1031-B28 to A.P.), the Human Frontier Science Program (HFSP) Career Development Award (CDA00066/2015 to A.P.), the SFB RNA-Deco (project number F 80 to A.P.) and EMBO-YIP funds (to A.P.). L.L.-O. was supported by an SNF Early Postdoc Mobility fellowship (P2GEP3_191204) and an EMBO long-term fellowship (ALTF 1165-2019); K.R.G. was supported by a DOC Fellowship from the Austrian Academy of Sciences. We acknowledge the cryo-electron microscopy facility CEITEC MU of CIISB, Instruct-CZ Centre, supported by MEYS CR (LM2018127), and Diamond Light Source for granting us access and support at the cryo-EM facilities at the UK's national Electron Bioimaging Centre (eBIC) under proposal EM BI25222, funded by the Wellcome Trust, MRC and BBRSC. For the purpose of Open Access, the authors have applied a CC BY public copyright license to any Author Accepted Manuscript (AAM) version arising from this submission.

Author contributions. F.L., L.L.-O., D.H. and A.P. conceived the study; F.L. performed most experiments with the help of C.P.; L.L.-O. obtained the final cryo-EM maps and modeled all ribosomes with contributions of F.L., A.M. and D.H. and help from S.K.; I.G. prepared and screened grids, collected EM data, and together with F.L. and D.H., contributed to the initial cryo-EM data processing with help from K.B.; M.M. and E.R. performed and analyzed the crosslinking sample preparation, and K.M. supervised mass-spectrometry experiments and analyzed the crosslinking samples; T.-Y.L. provided Xenopus eggs and $24 \mathrm{hpf}$ embryos; D.H. performed the cryoDRGN analysis and F.L. and L.L-O. compared the resulting maps; A.P. and D.H. supervised the project; L.L.-O., F.L., and A.P. wrote the manuscript with input from all authors.

Competing interests. The authors declare no competing interests.

Materials \& Correspondence. Correspondence and requests for materials should be addressed to A.P. 


\section{Extended Data Figures \& Tables}

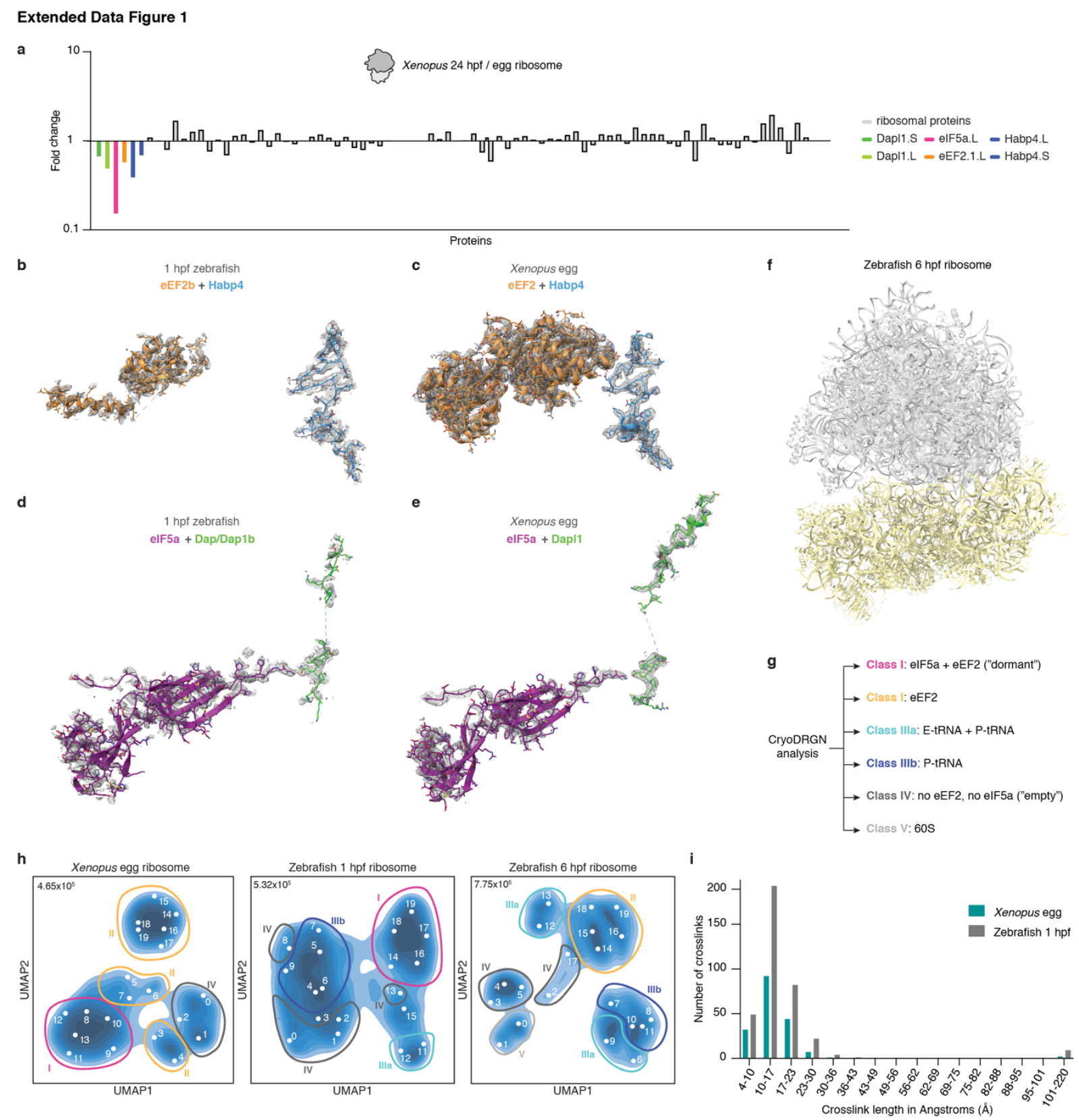

Extended Data Fig. 1. Characterization of the dormant ribosome state in zebrafish $1 \mathrm{hpf}$ and Xenopus egg ribosomes. a, Fold change of ribosome-associated factors and core ribosomal proteins after comparing mass spectrometry data of purified ribosomes from unfertilized Xenopus eggs and 24 hpf larvae (stage 14) (n=1). b-e, Densities of the two modules, namely Habp4-eEF2b/eEF2 (b-c), and Dap1b/Dapl1-eIF5a (d-e), that are characteristic for dormant ribosomes in zebrafish 1 hpf embryos and Xenopus eggs. f, Overview of the ribosome structure isolated from 6 hpf zebrafish embryos lacking ribosome-associated factors. $\mathbf{g}$, Classes assigned to the volumes obtained after the analysis of ribosomal particle heterogeneity using cryoDRGN. $\mathbf{h}$, Latent space representations of ribosomal particles from Xenopus eggs (left), $1 \mathrm{hpf}$ zebrafish embryos (middle) and $6 \mathrm{hpf}$ zebrafish embryos (right) as UMAP embeddings after training a cryoDRGN latent variable model. Classes are depicted with circles in Roman numbers, map volumes are indicated with Arabic numbers. Total particle numbers are shown on the top left of each graph. $\mathbf{i}, \mathrm{C} \alpha-\mathrm{C} \alpha$ distance distribution of the DSSO-induced crosslinks identified in Xenopus egg and zebrafish $1 \mathrm{hpf}$ ribosomes. 
bioRxiv preprint doi: https://doi.org/10.1101/2021.11.03.467131; this version posted November 3, 2021. The copyright holder for this

preprint (which was not certified by peer review) is the author/funder, who has granted bioRxiv a license to display the preprint in perpetuity. It is made available under aCC-BY-NC 4.0 International license.

\section{Extended Data Figure 2}
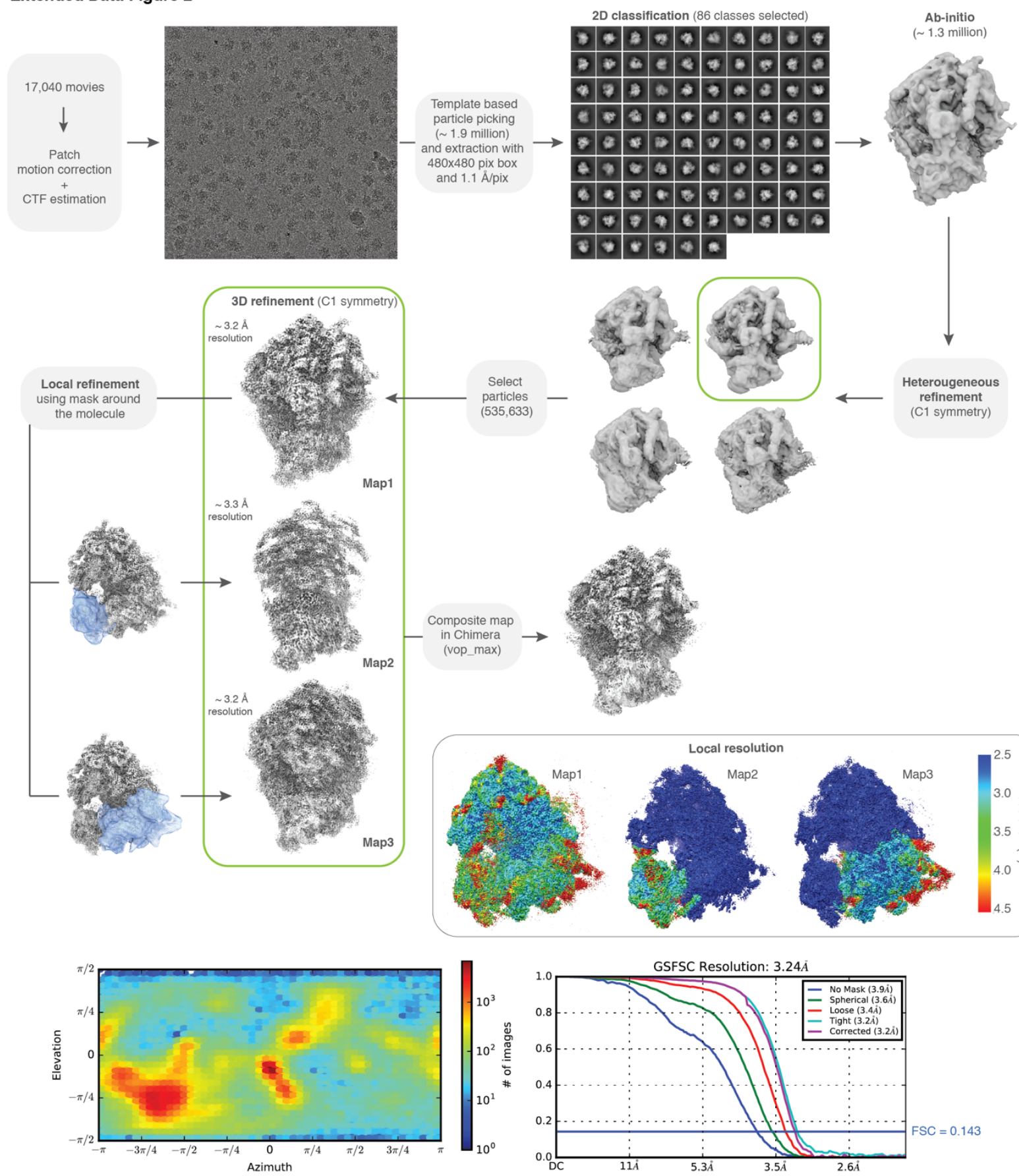

Heterougeneous refinement
(C1 symmetry)
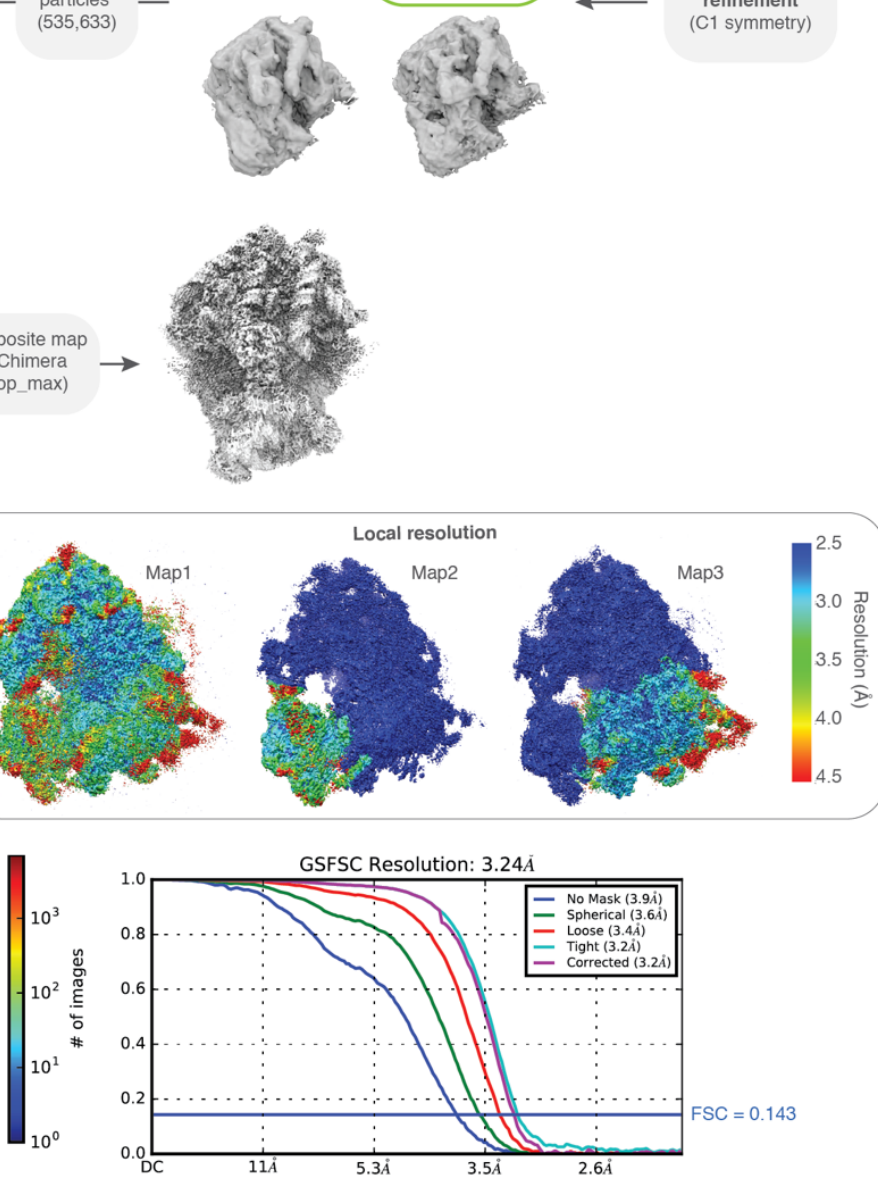

Extended Data Fig. 2. Processing pipeline of the $1 \mathrm{hpf}$ zebrafish ribosome. All steps were done in Cryosparc v3.2.0. Maps are shown in gray, masks in blue. The orientation distribution plot for all particles contributing to Map1 and the Gold-Standard Fourier Shell Correlation (GSFSC) of the respective map is shown on the bottom. Local resolution maps were calculated for Map1, Map2 and Map3. 
bioRxiv preprint doi: https://doi.org/10.1101/2021.11.03.467131; this version posted November 3, 2021. The copyright holder for this preprint (which was not certified by peer review) is the author/funder, who has granted bioRxiv a license to display the preprint in perpetuity. It is made available under aCC-BY-NC 4.0 International license.

\section{Extended Data Figure 3}

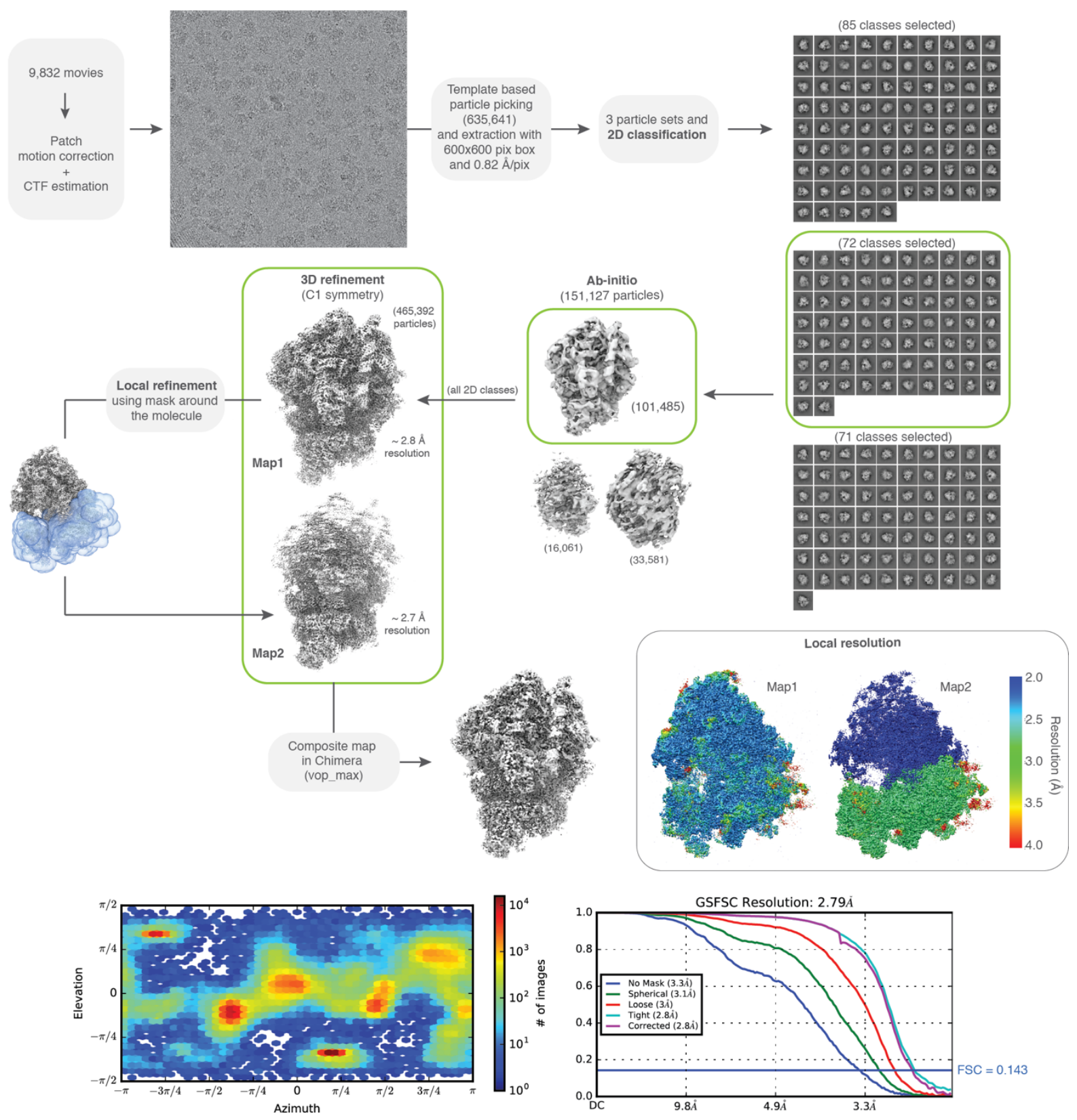

Extended Data Fig. 3. Processing pipeline of the Xenopus egg ribosome. All steps were done in Cryosparc v3.2.0. Maps are shown in gray, masks in blue. The orientation distribution plot for all particles contributing to Map1 and the Gold-Standard Fourier Shell Correlation (GSFSC) of the respective map is shown on the bottom right. Local resolution maps were calculated for Map1 and Map2. 
bioRxiv preprint doi: https://doi.org/10.1101/2021.11.03.467131; this version posted November 3, 2021. The copyright holder for this

preprint (which was not certified by peer review) is the author/funder, who has granted bioRxiv a license to display the preprint in perpetuity. It is made available under aCC-BY-NC 4.0 International license.

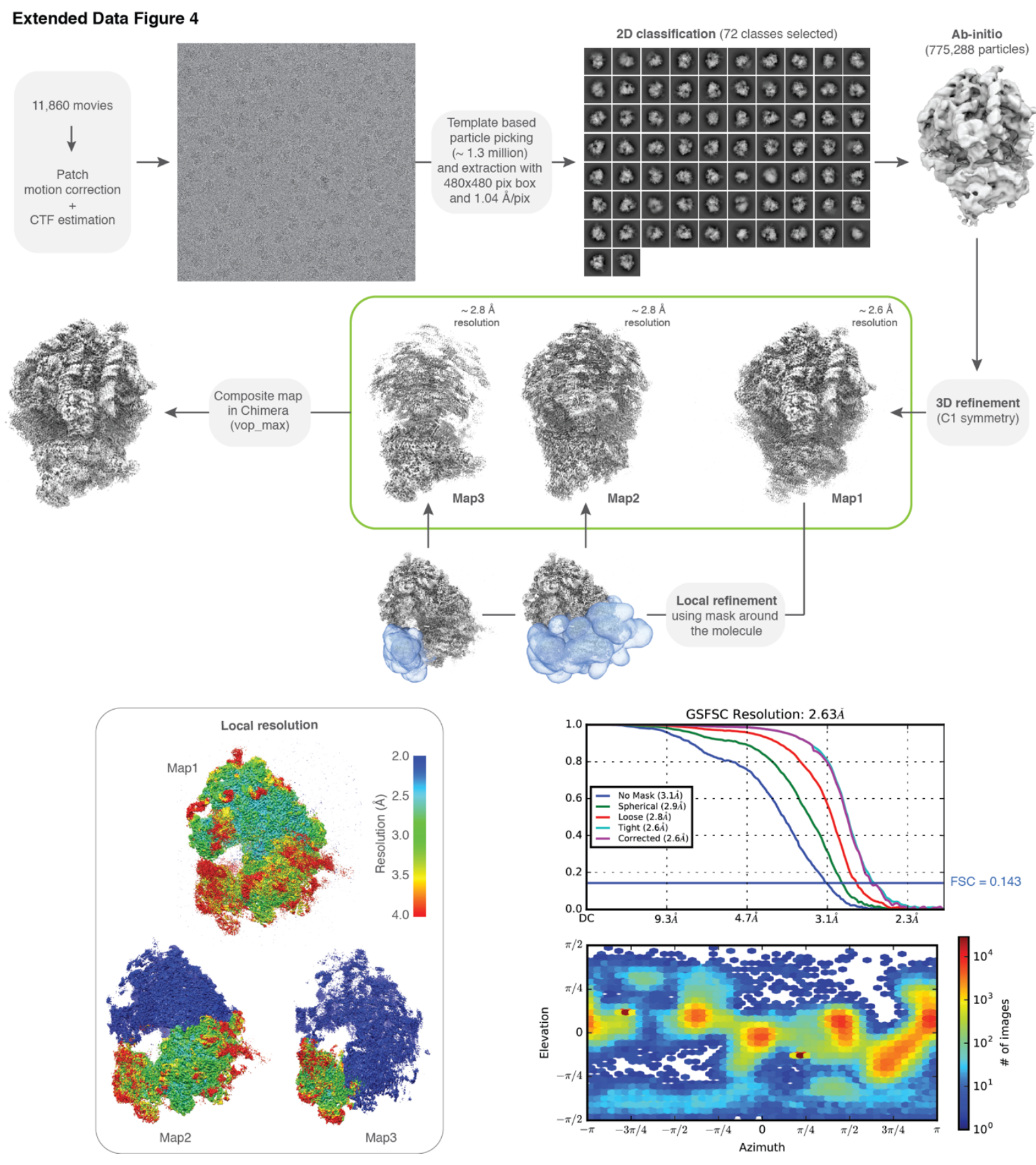

Extended Data Fig. 4. Processing pipeline of the $6 \mathrm{hpf}$ zebrafish ribosome. All steps were done in Cryosparc v3.2.0. Maps are shown in gray, masks in blue. The orientation distribution plot for all particles contributing to Map1 and the Gold-Standard Fourier Shell Correlation (GSFSC) of the respective map is shown on the bottom-right. Local resolution maps were calculated for Map1, Map2 and Map3. 


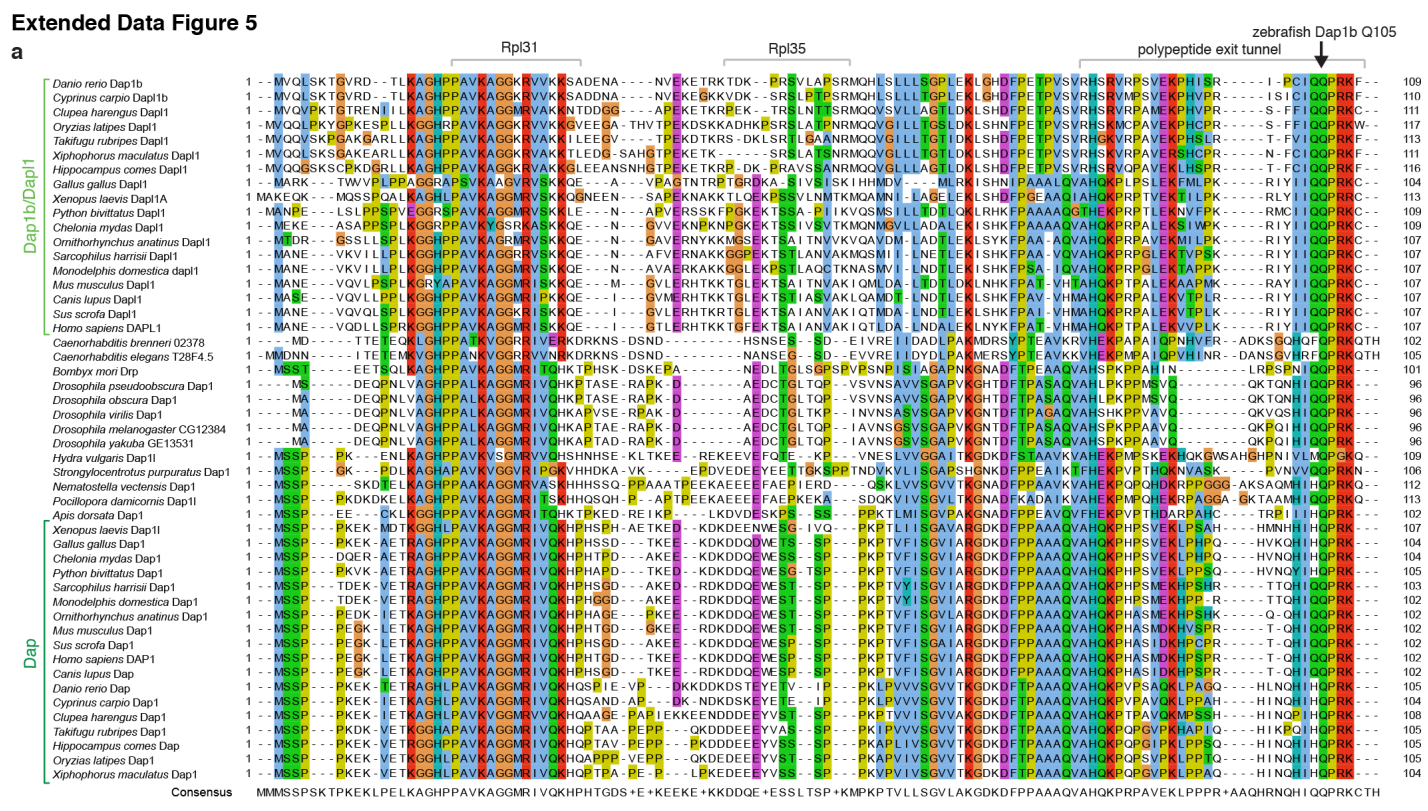

b

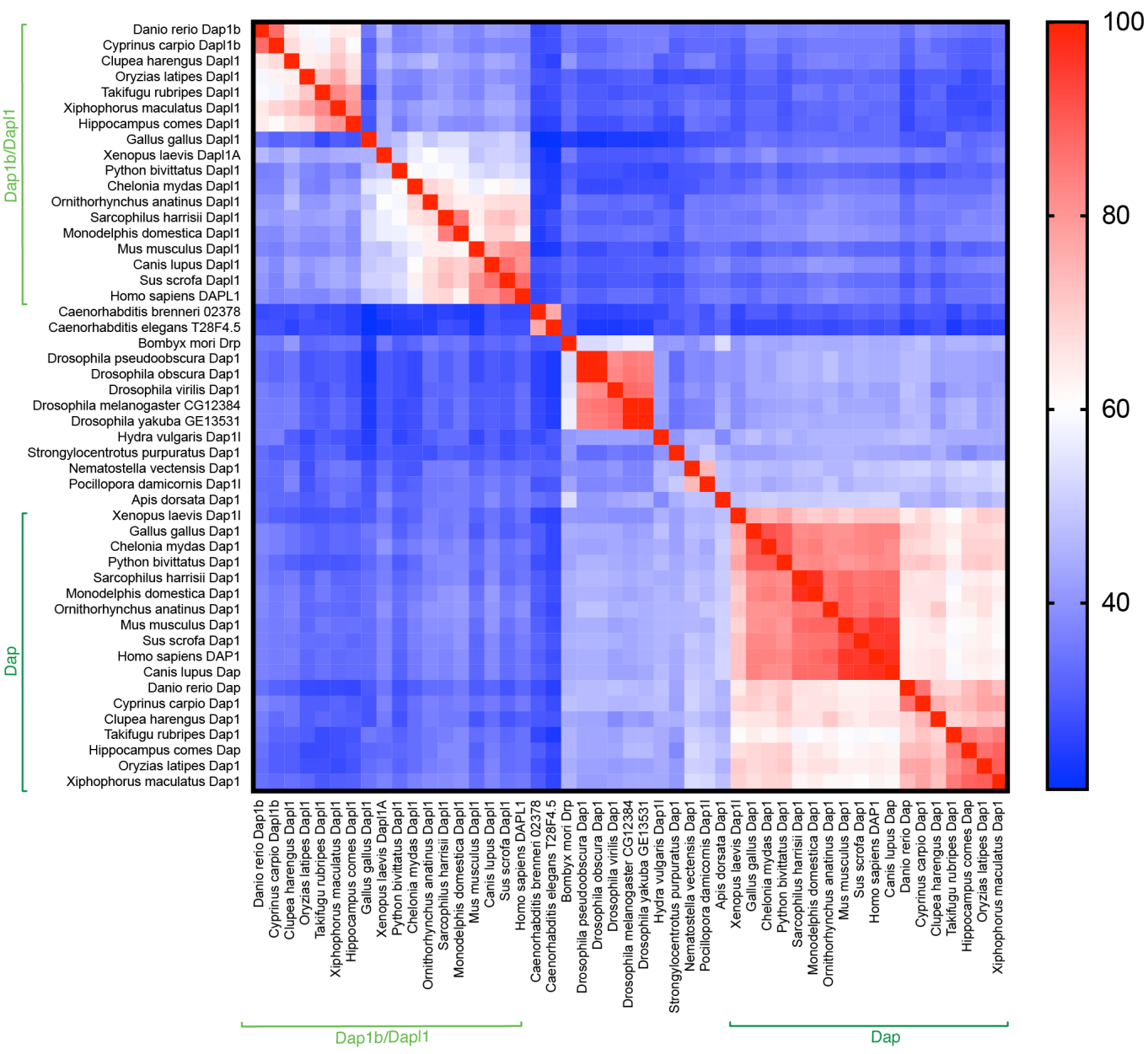

Extended Data Fig. 5. Analysis of amino acid sequence conservation of the Dap protein family. a, Protein sequence alignment illustrates sequence motifs shared between all Dap family proteins. Vertebrates have two paralogues, namely Dap1b/Dapl1 (light green) and Dap (dark green). Invertebrates only encode one homolog (Dap1) that clusters in between Dap1b/Dap11 and Dap proteins. b, Percentage identity matrix illustrating the homology of Dap1b/Dapl1 (light green), Dap (dark green) and ancestral Dap1 proteins across different species. Red, 100\% sequence identity; blue, 25\% sequence identity. 
a
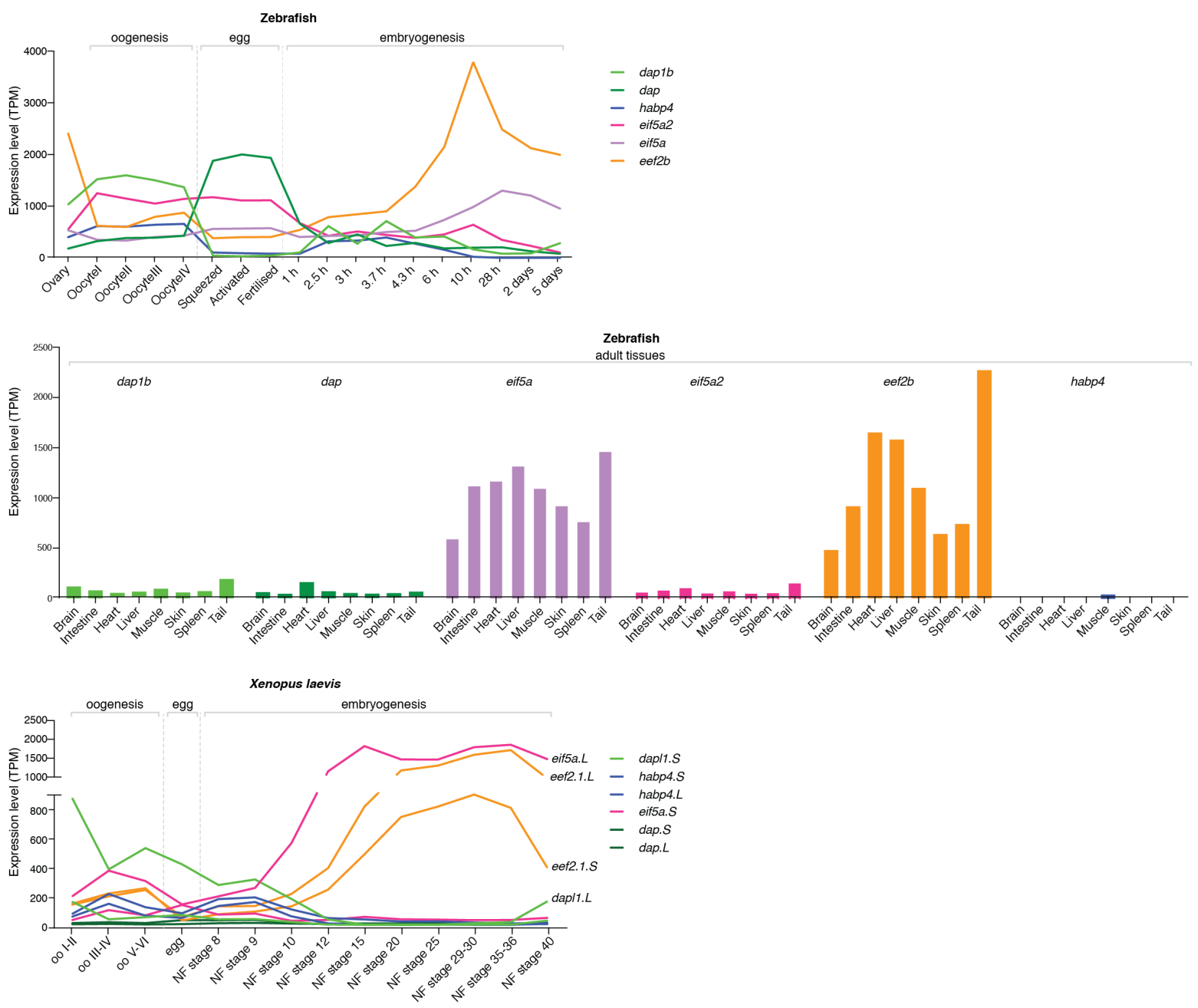

Extended Data Fig. 6. RNA expression of ribosome-associated factors during oogenesis, egg stages, embryo development and across adult tissues in zebrafish and Xenopus. (A) mRNA expression levels of zebrafish eif5a/eif5a2 (purple), eef2 (orange), dap1b/dap (green) and habp4 (blue). PolyA+ RNA-seq during zebrafish oogenesis (oocyte stages I-IV), egg-stages (un-activated, activated, fertilized) and embryogenesis ( $2-4$ cell, 256 cell, 1000 cell, oblong, dome, shield, bud, 1 day, 2 days, 5 days post-fertilization ${ }^{24,85}$; TPM, transcripts per million. $\mathbf{b}$, mRNA expression levels of ribosomeassociated factors in zebrafish adult tissues. Amongst all the tissues tested, only general translation factors eif5 $a$ and eef $2 b$ transcripts are highly expressed in adult tissues. TPM, transcripts per million. c, mRNA expression levels of all paralogs of Xenopus eif5a, eef2, dap, dapll and habp 4 derived from riboMinus-seq data ${ }^{86}$. TPM, transcripts per million. 

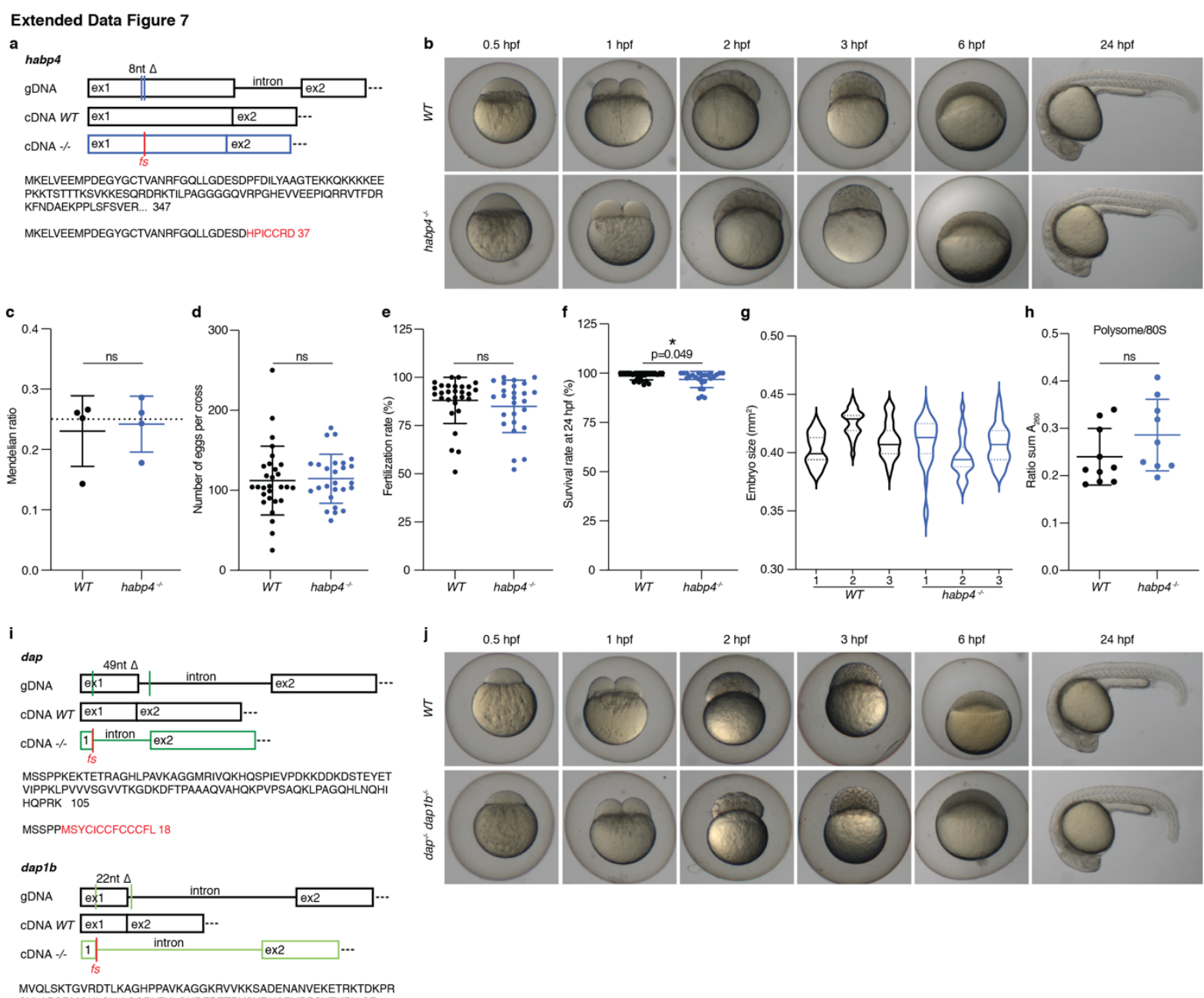

MVQLSKTGVRDTLKAGHPPAVKAGGKRVVKKSADENANVEKETRKTDKP SVLAPSRMOHLSL
PCIQQPRKF 109

MVQLSKTGVRDTLKAGFRRI 20
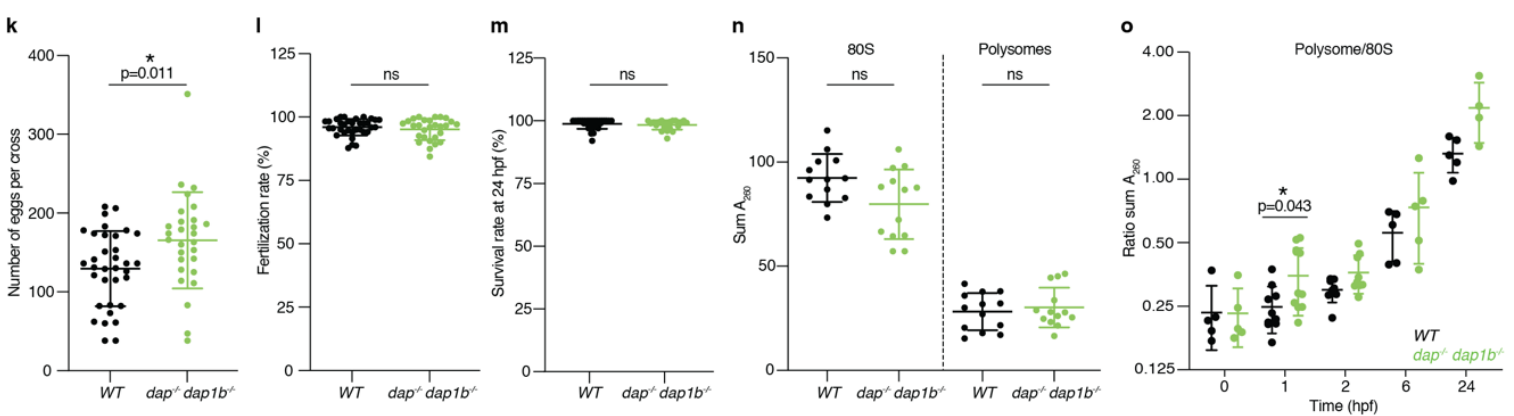

Extended Data Fig. 7. Characterization of habp ${ }^{-/}$and dap $^{-/}$, dap1 $b^{-/}$zebrafish mutants. a, Scheme showing a deletion of 8 nucleotides in exon 1 of habp 4 which leads to a truncated protein of 37 amino acids. b, Early development of habp $4^{-/}$embryos compared to wild type (WT). c, Mendelian ratio of finclips from a cross of $h a b p 4^{+-}$parents $\left(25 \%\right.$ of habp $4^{-/-}$and $W T$ fish are expected (dotted line), $\mathrm{n}=4$ crosses). d, Number of eggs laid per $W T$ ( $\mathrm{n}=29$ crosses) or habp $4^{-/}$( $\mathrm{n}=26$ crosses) female. e, Fertilization rates of $W T$ ( $\mathrm{n}=29$ crosses) and habp $4^{-/}(\mathrm{n}=26$ crosses $)$ fish. $\mathbf{f}$, Survival rate of $W T(\mathrm{n}=29$ crosses) and habp $4^{-/}$( $\mathrm{n}=25$ crosses) 24 hpf embryos. g, Size (in square millimeters) of WT and habp $4^{-}$ - 6 hpf embryos obtained after setting up 3 different pairs of fish for mating (X axis). Significant differences in size ( $p$-value $<0.05$, Kruskal-Wallis test) occur within $\left(2 / 3 \mathrm{WT}, 1 / 3 \mathrm{habp}^{-/}\right)$and between genotypes (4/9 comparisons) ( $n=31,22$ and 35 embryos for $W T$ pairs 1-3, respectively; $n=36,24$ and 33 embryos for habp $4^{-/}$pairs 1-3, respectively). $\mathbf{h}$, Quantification of polysome-to-monosome ratios of polysome gradients from $W T$ and $h a b p 4^{-/-} 1 \mathrm{hpf}$ embryos $(\mathrm{n}=9)$. i, Scheme showing deletions in exon 1 and intron 1 of dap (49 nucleotides) and daplb (22 nucleotides) leading to truncated proteins of 18 
(Dap) and 20 (Dap1b) amino acids. j, Early embryo development of $\mathrm{dap}^{-/-}$, dap $1 b^{-/-}$mutants compared to $W T$. k, Number of eggs laid by $W T$ ( $\mathrm{n}=34$ crosses) or $\operatorname{dap}^{-/}$, dap $1 b^{-/}(\mathrm{n}=28$ crosses) fish. $\mathbf{l}$, Fertilization rates of $W T(\mathrm{n}=34$ crosses $)$ and $\operatorname{dap}^{-/}$, dap $1 b^{-/-}(\mathrm{n}=28$ crosses $)$ fish. $\mathbf{m}$, Survival rate of $W T$ $(\mathrm{n}=28$ crosses $)$ and dap $^{-/},{\text {dap } 1 b^{-/-}}(\mathrm{n}=21$ crosses $) 24 \mathrm{hpf}$ embryos. $\mathbf{n}$, Sum of $\mathrm{A}_{260}$ in polysome gradient analyses of monosomes and polysomes $(\mathrm{n}=13)$ of $W T$ and $d a p^{-/}$, daplb $b^{-/} 1 \mathrm{hpf}$ embryos. o, Quantification of polysome-to-monosome ratios (in $\log 2$ scale) at different stages of development of $W T$ and dap $^{-/}$, dap $1 b^{-/-}$eggs and embryos ( 0 h: $\mathrm{n}=5 ; 1$ hpf: $\mathrm{n}=10 ; 2$ hpf: $\mathrm{n}=8 ; 6$ hpf: $\mathrm{n}=5 ; 24$ hpf: $\left.\mathrm{n}=4\right)$. Data in (c-f, h, k-o) are represented as scatter dot plots with means and SD. Data in g are represented as violin plot with median and quartiles. Significance was determined using Mann-Whitney test (c-f, h, $\mathrm{k}-\mathrm{o}$ ) or Kruskal-Wallis test $(\mathrm{g}) . \mathrm{ns}=$ not significant, ${ }^{*} p$-value $<0.05$, hpf $=$ hours post fertilization, $\mathrm{fs}=$ frameshift. 

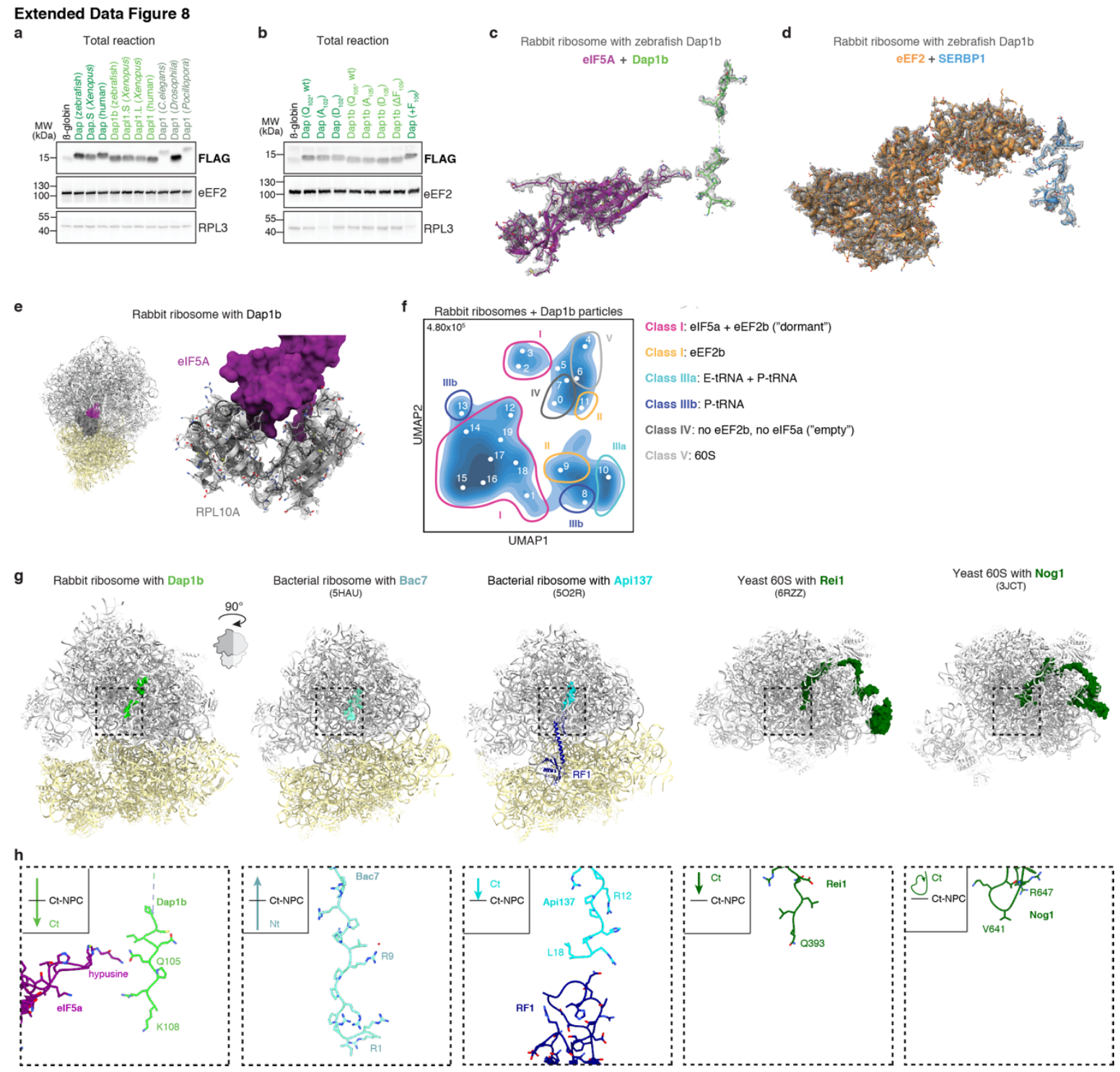

Extended Data Fig. 8. Recombinant Dap1b binds to the polypeptide exit tunnel of rabbit ribosomes. a, Western blot of the total in vitro translation reaction shown in Fig. 4D. b, Western blot of the total in vitro translation reaction shown in Fig. 4F. c-d, Densities of eIF5A and zebrafish Dap1b (c), and eEF2 and SERBP1 (d) modules in the rabbit ribosome with recombinant zebrafish Dap1b. e, The L1 stalk protein RPL10A (in gray) interacts with eIF5A (in dark magenta) in the rabbit ribosome with recombinant zebrafish Dap1b. An overview of the RPL10A position on the rabbit ribosome is shown on the left; RPL10A density is shown in mesh (right). f, Latent space representation of ribosomal particles of the dataset containing rabbit ribosomes with recombinant zebrafish Dap1b protein as a UMAP embedding after training a cryoDRGN latent variable model. Classes are depicted with circles in Roman numbers, map volumes are indicated with Arabic numbers. Total particle number is shown on the top left of the graph. g, Structures of ribosomes with proteins and peptides inserted into the polypeptide exit tunnel. From left to right: zebrafish Dap1b, Bac7 (5HAU $\left.{ }^{16}\right)$, Api137 $\left(5 \mathrm{O} 2 \mathrm{R}^{57}\right)$, Rei1 $\left(6 \mathrm{RZZ}^{58}\right)$ and $\operatorname{Nog} 1\left(3 \mathrm{JCT}^{61}\right)$. Models were superimposed using the command mmaker in ChimeraX and clipped to have a better view of the polypeptide exit tunnel. Boxed areas (dashed boxes) are shown at higher magnification in H. h, Detail of the peptidyl-transferase center (PTC) of the ribosomes shown in G. Schemes on the top-left of each box illustrate the relative position of the factor within the PTC in relation to the $\mathrm{C}$-terminal residue of the nascent peptide chain (NPC). $\mathrm{Nt}$ refers to $\mathrm{N}$-terminus, $\mathrm{Ct}$ corresponds to C-terminus. 
bioRxiv preprint doi: https://doi.org/10.1101/2021.11.03.467131; this version posted November 3, 2021. The copyright holder for this preprint (which was not certified by peer review) is the author/funder, who has granted bioRxiv a license to display the preprint in perpetuity. It is made available under aCC-BY-NC 4.0 International license.

\section{Extended Data Figure 9}
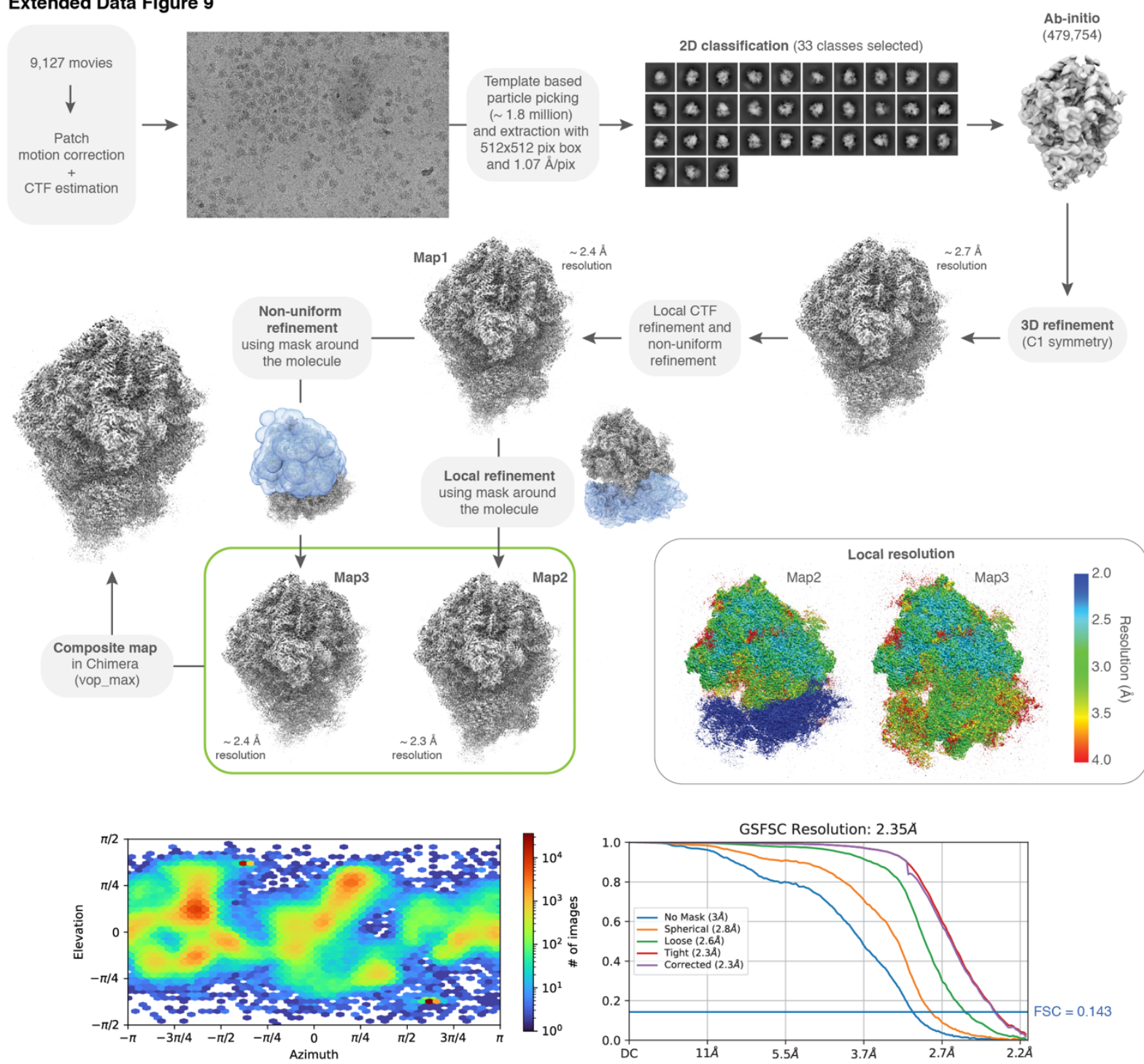

Extended Data Fig. 9. Processing pipeline of the rabbit ribosome with recombinant zebrafish Dap1b. Processing pipeline for obtaining an 80 S density map of the rabbit ribosome with Dap $1 \mathrm{~b}$. Local resolution maps were calculated for Map2 and Map3. The orientation distribution plot for all particles contributing to Map1 and the Gold-Standard Fourier Shell Correlation (GSFSC) of the respective map is shown on the bottom. 
bioRxiv preprint doi: https://doi.org/10.1101/2021.11.03.467131; this version posted November 3, 2021. The copyright holder for this

preprint (which was not certified by peer review) is the author/funder, who has granted bioRxiv a license to display the preprint in perpetuity. It is made available under aCC-BY-NC 4.0 International license.

Extended Data Table 1. Cryo-EM data collection and refinement statistics of the ribosome structures presented in this study.

\begin{tabular}{|c|c|c|c|c|}
\hline & 1 hpf zebrafish & 6 hpf zebrafish & Xenopus egg & Rabbit + Dap1b \\
\hline PDB ID & $7 \mathrm{OYA}$ & 7OYB & $7 \mathrm{OYC}$ & 7OYD \\
\hline \multicolumn{5}{|l|}{ Data collection } \\
\hline Microscope & \multicolumn{4}{|c|}{ Thermo Fisher Titan Krios } \\
\hline Acceleration Voltage $(\mathrm{kV})$ & \multicolumn{4}{|c|}{300} \\
\hline Detector & Falcon $3 \mathrm{EC}$ & Falcon $3 \mathrm{EC}$ & Falcon $3 \mathrm{EC}$ & K3 \\
\hline Magnification (nominal) & 81.000 & 81.000 & 105.000 & 81.000 \\
\hline Defocus range $(\mu \mathrm{m})$ & $0.5-4$ & $0.5-3$ & $0.2-2$ & $0.4-3.2$ \\
\hline Calibrates pixel size $(\AA / p x)$ & 1.1 & 1.04 & 0.82 & 1.07 \\
\hline Electron exposure $\left(\mathrm{e}^{-} / \AA^{2}\right)$ & 43 & 48 & 40 & 50 \\
\hline Exposure rate $\left(\mathrm{e}^{-} / \AA^{2} / \mathrm{s}\right)$ & 43 & 48 & 40 & 50 \\
\hline Number of frames per movie & 39 & 39 & 39 & 41 \\
\hline Collection software & Cryosparc 3.2 .0 & Cryosparc 3.2 .0 & Cryosparc 3.2 .0 & Cryosparc 3.2 .0 \\
\hline Number of micrographs & 17,040 & 11,860 & 9,832 & 9,127 \\
\hline Initial particle number & $1,961,364$ & $1,285,670$ & 635,641 & $1,808,120$ \\
\hline Final particle number & 535,633 & 775,288 & 465,392 & 479,754 \\
\hline Map resolution $(\AA, F S C=0.143)$ & 3.2 & 2.6 & 2.8 & 2.4 \\
\hline \multicolumn{5}{|l|}{ Refinement } \\
\hline Software & Phenix 1.17.1 & Phenix 1.17.1 & Phenix 1.17.1 & Phenix 1.17.1 \\
\hline Initial model(s) & $\begin{array}{l}\text { 4UG0 } \\
6 \mathrm{MTE} \\
5 \mathrm{DAT} \\
\end{array}$ & 4UG0 & $\begin{array}{l}\text { 4UG0 } \\
6 \mathrm{MTE} \\
5 \mathrm{DAT} \\
\end{array}$ & $\begin{array}{l}6 \mathrm{MTE} \\
5 \mathrm{DAT} \\
5 \mathrm{GAK} \\
\end{array}$ \\
\hline Correlation coefficient $\left(\mathrm{CC}_{\text {mask }}\right)$ & 0.83 & 0.84 & 0.82 & 0.76 \\
\hline Map sharperning factor $\left(\AA^{2}\right)$ & -108.5 & -89.2 & -73.4 & -50.2 \\
\hline Model composition (chains) & 82 & 77 & 81 & 85 \\
\hline Non-hydrogen atoms & 199,914 & 194,870 & 198,952 & 213,014 \\
\hline Protein residues & 11,179 & 10,795 & 11,387 & 12,293 \\
\hline nucleotides & 5,130 & 5,035 & 5,006 & 5,316 \\
\hline Ligands & $\begin{array}{l}\text { MG: } 202 \\
\mathrm{ZN}: 7\end{array}$ & $\begin{array}{l}\text { MG: } 203 \\
\text { ZN: } 7\end{array}$ & $\begin{array}{l}\text { MG: } 134 \\
\mathrm{ZN}: 7\end{array}$ & $\begin{array}{l}\text { ZNA: } 1 \\
\text { MG: } 201 \\
\text { B8N: } 1 \\
\text { ZNK: } 1 \\
\text { ZN: } 5 \\
4 A C: 2\end{array}$ \\
\hline \multicolumn{5}{|l|}{ B factors $\left(\AA^{2}, \min / \max /\right.$ mean $)$} \\
\hline Protein & $4.4 / 281.9 / 53.3$ & $12.8 / 350.6 / 51.7$ & $4.4 / 300.2 / 53.4$ & $4.4 / 244.8 / 82.7$ \\
\hline RNA & $13.9 / 234.1 / 53.1$ & $13.9 / 205.7 / 56.6$ & $13.9 / 205.7 / 54.6$ & $20.0 / 333.2 / 81.7$ \\
\hline Ligands & $7.8 / 102.4 / 28.4$ & $7.8 / 102.4 / 27.0$ & $7.8 / 102.4 / 25.1$ & $25.8 / 120.5 / 57.1$ \\
\hline \multicolumn{5}{|l|}{ RMS deviations } \\
\hline Bond length $(\AA)$ (\#>4 sigma) & 0.003 & 0.003 & 0.003 & 0.005 \\
\hline Bond angle $\left({ }^{\circ}\right)(\#>4$ sigma $)$ & 0.620 & 0.614 & 0.619 & 0.714 \\
\hline \multicolumn{5}{|l|}{ Validation } \\
\hline MolProbity score & 1.59 & 1.72 & 1.54 & 1.68 \\
\hline Clashscore & 6.04 & 8.01 & 6.95 & 6.96 \\
\hline Poor Rotamers (\%) & 1.30 & 1.32 & 0.76 & 1.06 \\
\hline $\mathrm{C} \beta$ deviations $(\%)$ & 0.01 & 0.00 & 0.00 & 0.01 \\
\hline CABLAM outliers (\%) & 2.35 & 2.36 & 2.27 & 2.25 \\
\hline Favored (\%) & 97.03 & 96.86 & 97.10 & 96 \\
\hline Allowed (\%) & 2.97 & 3.14 & 2.90 & 4 \\
\hline Disallowed (\%) & 0 & 0 & 0 & 0 \\
\hline
\end{tabular}


bioRxiv preprint doi: https://doi.org/10.1101/2021.11.03.467131; this version posted November 3, 2021. The copyright holder for this

preprint (which was not certified by peer review) is the author/funder, who has granted bioRxiv a license to display the preprint in perpetuity. It is made available under aCC-BY-NC 4.0 International license.

Extended Data Table 2. Classification of the map volumes obtained with CryoDRGN.

\begin{tabular}{|c|c|c|c|c|c|c|c|c|c|c|c|c|c|c|c|}
\hline Volume & $\frac{1}{\text { open }}$ & $\begin{array}{l}\text { Position of L1 st } \\
\text { half-closed }\end{array}$ & alk & eEF2 & elF5a & Pa294/Ebp1 & E site & $\begin{array}{l}\text { tRNA } \\
\text { P site }\end{array}$ & A site & ssu & Other comments & \begin{tabular}{|l} 
Nr. of particles \\
\end{tabular} & $\%$ of particles & Cluster size in $\%$ & Cluster \\
\hline & & & & & & & & & ribosome & & & & & \multirow{4}{*}{16.59} & \\
\hline $\begin{array}{l}\text { vol 000.mrc } \\
\end{array}$ & & + & & Z & - & & & & & + & & 28093 & 6.04 & & \multirow{3}{*}{ IV } \\
\hline vol 001.mrc & & + & - & & & & & & & + & & 30082 & 6.46 & & \\
\hline vol_002.mrc & & + & & & & & & & & + & & 19046 & 4.09 & & \\
\hline vol 003 .mre & & + & - & + & & & & & & + & & 18609 & 4.00 & \multirow{4}{*}{19.90} & \multirow{4}{*}{ II } \\
\hline $\begin{array}{l}\text { vol_1004.mre } \\
\text { vol 1005 mrc }\end{array}$ & & + & + & + & 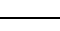 & 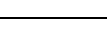 & & & & + & & 24807 & $\frac{5.33}{2.39}$ & & \\
\hline vol 006 .minc & & & $\frac{t}{+}$ & $\frac{t+}{++}$ & - & & & & & $\frac{t}{+}$ & & $\frac{13471}{12591}$ & $\frac{2.89}{2.71}$ & & \\
\hline vol_007.mrc & & & + & +++ & & & & & & + & & 23124 & 4.97 & & \\
\hline vol_008.mrc & & & + & +++ & + & & & & & & & 26148 & 5.62 & & \\
\hline vol 009.mrc & & & + & +++ & + & & & & & + & & 32606 & 7.01 & & \\
\hline $\begin{array}{l}\text { vol [1010.mre } \\
\text { vol 011 merc }\end{array}$ & & & $\begin{array}{c}+ \\
+\end{array}$ & $\frac{+++}{+++}$ & $\begin{aligned}++ \\
++t\end{aligned}$ & + & & & & ++ & -5 & 32797 & $\frac{7.05}{7.51}$ & 40.18 & I \\
\hline vol 012 .mrc & & & $\frac{t}{+}$ & $\frac{t++}{++t}$ & +++ & & & & & $\begin{array}{ll}+ \\
+ \\
+\end{array}$ & & $\frac{34951}{30543}$ & $\frac{7.51}{6.56}$ & & \\
\hline vol_013.mrc & & & + & +++ & + & & & & & + & & 29944 & $\frac{0.00}{6.43}$ & & \\
\hline vol_014.mrc & & + & & ++ & & & & & & + & & 18960 & 4.07 & & \\
\hline vol_015.mrc & & + & & ++ & & & & & & + & & 18361 & 3.95 & & \\
\hline vol 016.mrc & & + & & ++ & & & & & & + & & 17420 & 3.74 & 23.33 & II \\
\hline vol $017 . \mathrm{mrc}$ & & + & & ++ & & & & & & + & & 18218 & 3.91 & & 11 \\
\hline vol_018.mrc & & + & & ++ & & & & & & + & & 15890 & 3.41 & & \\
\hline $\begin{array}{l}\text { vol 019.mrc } \\
\end{array}$ & & + & & ++ & & & & & & + & & 19731 & 4.24 & & \\
\hline & & & & & & & & afish $1 \mathrm{~h}$ & f ribosome & & & & & & \\
\hline vol_000.mrc & + & & & & & +++ & & & & + & ES27 & 29338 & 5.48 & & \\
\hline vol_001.mrc & + & & & & & +++ & & & & + & ES27 & 29341 & 5.48 & 16.44 & IV \\
\hline vol 002.mrc & + & - & & & & +++ & & & & + & ES27 & 29363 & 5.48 & & \\
\hline vol _003.mrc & + & & 二 & 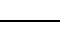 & & $\frac{+++}{+++4}$ & & weak & & + & $\begin{array}{l}\text { ES27 } \\
\text { ESS7 }\end{array}$ & 29341 & $\frac{6.06}{554}$ & & n.a. \\
\hline $\begin{array}{l}\text { vol_004.mre } \\
\text { vol 1005.mrc }\end{array}$ & & $+\frac{+}{+}$ & 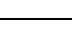 & 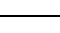 & & $\frac{t++}{+++}$ & \begin{tabular}{|l} 
weak \\
weak
\end{tabular} & & & $\begin{array}{c}+ \\
+\end{array}$ & $\frac{\mathrm{ES} 27}{\mathrm{E} 227}$ & 296900 & $\frac{5.54}{4.74}$ & & \\
\hline vol_006.mrc & & + & & 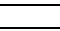 & & +++ & weak & & & + & $\begin{array}{ll}\text { ES27 } \\
\end{array}$ & 31536 & $\frac{4.14}{5.89}$ & 21.85 & IIIb \\
\hline vol_007.mrc & & + & & & & ++++ & + & & & + & ES27 & 30429 & 5.68 & & \\
\hline$\frac{\text { vol } 008 . \mathrm{mrc}}{\text { vol 1009.mrc }}$ & + & + & & & & $\frac{+}{+}$ & weak & & & $+\frac{+}{+}$ & $\begin{array}{ll}\mathrm{ES} 27 \\
\end{array}$ & 22558 & 4.21 & 4.21 & IV \\
\hline 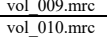 & + & & & & & $\begin{array}{c}+ \\
+ \\
\end{array}$ & weak & & & $\frac{+}{+}$ & $\mathrm{ES21}$ & $\begin{array}{l}25 / 53 \\
19789\end{array}$ & $\frac{4.81}{3.69}$ & $\frac{4.81}{3.69}$ & $\frac{1 \mathrm{IIb}}{\mathrm{IV}}$ \\
\hline vol 011 .mre & & & + & -5 & & + & +++ & +++ & & + & & 20850 & 3.89 & & \\
\hline vol_012.mrc & & & + & - & 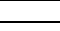 & + & +++ & +++ & & + & & 20567 & 3.84 & 7.73 & IIIIa \\
\hline $\begin{array}{l}\text { vol_013.mrc } \\
\end{array}$ & & & + & & & ++++ & & & & + & $\begin{array}{ll}\text { ES27 } \\
\end{array}$ & 15735 & $\begin{array}{l}.94 \\
1.77\end{array}$ & 2.94 & IV \\
\hline vol 014.mre & & & + & +++ & +++ & $4+++$ & 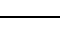 & & & + & $\begin{array}{l}\text { ES27 } \\
\text { E297 }\end{array}$ & $\frac{22868}{28797}$ & $\begin{array}{l}4.27 \\
5.38\end{array}$ & $\begin{array}{l}4.27 \\
5.38\end{array}$ & $\frac{1}{\text { n.a. }}$ \\
\hline vol _lis.mitc & & & + & +++ & +++ & ++ & & & & + & ES27 & $\frac{26776}{26676}$ & 4.98 & & n.... \\
\hline vol_017.mrc & & & + & +++ & +++ & ++ & & & & + & ES27 & 29496 & 5.51 & 2260 & I \\
\hline vol 018.mrc & & & + & +++ & +++ & ++ & & & & + & ES27 & 28085 & 5.24 & 22.02 & 1 \\
\hline vol 019.mrc & & & + & +++ & +++ & ++ & & & & + & ES27 & 36910 & 6.89 & & \\
\hline & & & & & & & & ish $6 \mathrm{~h}$ & & & & & & & \\
\hline vol_000.mrc & & 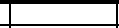 & + & 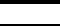 & - & & & & & 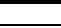 & $60 \mathrm{~S}$ & 37193 & 4.80 & & $\mathbf{v}$ \\
\hline vol 001.mrc & & & & & & & & & & & $60 \mathrm{~S}$ & 24601 & 3.17 & 7.97 & $v$ \\
\hline vol 002.mirc & & + & 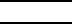 & 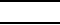 & & & & & & + & & $\begin{array}{l}24173 \\
\end{array}$ & 3.12 & & \\
\hline vol_003.mrc & + & & 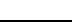 & 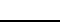 & & & & & & + & & 24249 & 3.13 & 14.55 & IV \\
\hline vol $004 . m r c$ & & + & - & & & & & & & + & & 37784 & 4.87 & 1400 & 10 \\
\hline 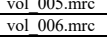 & & + & 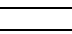 & & & + & $\begin{array}{l}\text { weak } \\
\end{array}$ & weak & & $\frac{+}{+}$ & & 33543 & 4.33 & 4.33 & IIIIa \\
\hline vol $007 . \mathrm{mrc}$ & & + & E & + & & + & weak & & & + & & 41943 & 5.41 & & \\
\hline vol_008.mrc & & + & 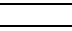 & + & & + & weak & & & + & & 39836 & 5.14 & 10.55 & $1 \mathrm{lltb}$ \\
\hline vol_009.mrc & & + & E & & & + & weak & weak & & + & & 43471 & 5.61 & & \\
\hline vol 01 & & + & & & & + & weak & weak & & + & & 37860 & 4.88 & & \\
\hline vol 011.mrc & & + & & & & + & weak & weak & & + & & 44216 & $\frac{5.70}{5}$ & 26.54 & IIIIa \\
\hline 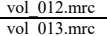 & & & $\frac{+}{+}$ & - & & & $\frac{+1+}{+1+}$ & $\frac{++4}{+4+}$ & & $\begin{array}{l}+ \\
+ \\
+\end{array}$ & & 39996 & $\frac{5.16}{5.19}$ & & \\
\hline vol_014.mrc & & & + & ++++ & & & & & & + & & 47238 & 6.09 & & \\
\hline vol__15.mrc & & + & & +++ & & 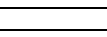 & & & & + & & 54283 & 7.00 & 19.99 & II \\
\hline vol 016.mrc & & & + & +++ & & & & & & + & & 53466 & 6.90 & & \\
\hline vol 017.mrc & & + & & & & & & & & + & & $\begin{array}{ll}466638 \\
23832\end{array}$ & $\frac{6.02}{3.07}$ & 6.02 & IV \\
\hline 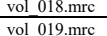 & & $\frac{+}{+}$ & - & $\frac{+++}{+++}$ & & - & & & & + & & $\frac{23832}{54148}$ & 3.07 & 10.06 & II \\
\hline & & & & & & & Rabbit ri & some wit & brafis! & Dap1b & & & & & \\
\hline $\begin{array}{l}\text { vol_000.mrc } \\
\end{array}$ & & & + & & & & & & & & & 9032 & 1.88 & 1.88 & $\overline{\text { IV }}$ \\
\hline vol $001 . \mathrm{mrc}$ & & & + & ++++ & +++ & + & - & & & + & & 13224 & 2.76 & & \\
\hline vol 002.mirc & & & + & +++ & + & + & & & & + & & 10158 & 2.12 & 6.50 & I \\
\hline vol_003.mrc & & 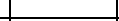 & & + & weak & +++ & & & & + & & 7788 & 1.62 & & \\
\hline vold & & & & & & +++ & & & & & $60 \mathrm{~S}$ & 17070 & 3.5 & 3.56 & $\mathbf{v}$ \\
\hline vol_- & & & + & + & + & ++ & & & & weak & & $\frac{159}{186}$ & $\frac{3 .}{3}$ & & n.a. \\
\hline vol_o & & & + & & 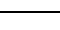 & & & & & + & $60 \mathrm{~s}$ & $\frac{18605}{15713}$ & $\frac{3.88}{3.28}$ & $\begin{array}{l}3.38 \\
3.28 \\
\end{array}$ & IV \\
\hline vol 008 .mrc & & + & & + & & + & + & & & + & & 27782 & 5.79 & 5.79 & IIIIb \\
\hline vol_005 & & & & +++ & 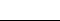 & ++++ & & & & + & & 32293 & 6.73 & 6.73 & II \\
\hline & & + & & ++ & & ++++ & +++ & ++4 & & + & & 39738 & 8.28 & 8.28 & IIIIa \\
\hline vol_0 & & + & & + & & ++ & & & & + & & 15488 & 3.2 & $\frac{23}{27-2}$ & II \\
\hline 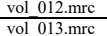 & & + & + & $\frac{+++}{++}$ & ++ & $\frac{+}{++}$ & weak & & & $\frac{+}{+}$ & & $\frac{15495}{20897}$ & $\frac{3.2}{4.3}$ & $\frac{3.23}{4.36}$ & $\frac{\mathrm{I}}{\mathrm{IIIb}}$ \\
\hline$\frac{v}{\text { vol } 014 . \mathrm{mrc}}$ & & & + & $\frac{t+4}{++t}$ & ++ & +7 & 年 & & & + & & 208697 & $\frac{4.36}{6.81}$ & & ilib \\
\hline $\mathrm{vol}^{015}$.mrc & & & + & $\frac{1+++}{+++}$ & ++ & + & & & & + & & $\begin{array}{ll}45784 \\
\end{array}$ & $\begin{array}{ll}9.54 \\
\end{array}$ & & \\
\hline vol _016.mrc & & & + & +++ & ++ & + & & & & + & & 48590 & 10.13 & 4596 & T \\
\hline vol _017.mrc & & & + & +++ & ++ & + & & & & + & & 41365 & 8.62 & 40.0 & 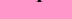 \\
\hline vol_018.mrc & & & + & ++++ & ++ & + & & & & + & & 32223 & 6.72 & & \\
\hline vol 019.mrc & & & + & ++++ & +++ & + & & & & + & & 19882 & 4.14 & & \\
\hline
\end{tabular}

Ribosome particles were classified into six clusters based on the presence or absence of eEF2, eIF5a and tRNAs, with class I particles ("dormant") containing eEF2 and eIF5A, class II particles containing only eEF2, class III particles containing either E and P tRNAs (IIIa) or a P tRNA (IIIb) and, in some cases, also eEF2; class IV particles ("empty") lacking eEF2, eIF5A and tRNAs, and class V particles containing only the large subunit (60S). Additional features, such as the position of the L1 stalk and the presence or absence of $\mathrm{Pa} 2 \mathrm{~g} 4 / \mathrm{Ebp} 1$, are also reported. Density values are qualitatively assessed with pluses, with one plus indicating poor density. Weak densities are reported as "weak". n.a.=non-assigned particles. U-maps depicting the position of the volumes in the clusters are shown in Extended Data Fig. $1 \mathrm{~h}$ (Xenopus and zebrafish) and Extended Data Fig. 8f (rabbit + zebrafish Dap1b). 
bioRxiv preprint doi: https://doi.org/10.1101/2021.11.03.467131; this version posted November 3, 2021. The copyright holder for this

preprint (which was not certified by peer review) is the author/funder, who has granted bioRxiv a license to display the preprint in perpetuity. It is made available under aCC-BY-NC 4.0 International license.

Extended Data Table 3. Crosslinks identified in ribosome samples from 1 hpf zebrafish embryos.

\begin{tabular}{|c|c|c|c|c|c|c|c|c|c|c|c|c|}
\hline \#CSMs & \#Proteins & Sequence $\mathbf{A}$ & Accession A & \begin{tabular}{c|}
$\begin{array}{c}\text { Position } \\
\text { A }\end{array}$ \\
\end{tabular} & Sequence B & Accession B & \begin{tabular}{c|}
$\begin{array}{c}\text { Position } \\
\text { B }\end{array}$ \\
\end{tabular} & $\begin{array}{c}\text { Protein } \\
\text { A }\end{array}$ & $\begin{array}{c}\text { Protein } \\
\text { B }\end{array}$ & $\begin{array}{c}\text { Best CSM } \\
\text { Score }\end{array}$ & $\begin{array}{c}\text { In } \\
\text { protein } \mathrm{A}\end{array}$ & $\begin{array}{c}\text { In } \\
\text { protein B }\end{array}$ \\
\hline 54 & 2 & DVHLA[K]HPELADK & Q7T1J9 & 6 & LLQ[K]QDGMDEDVVFR & B0R085 & 4 & Rps10 & Habp4 & 732.66 & 31 & 273 \\
\hline 21 & 1 & AVVIH[K]SK & B0R085 & 6 & LLQ[K]QDGMDEDVVFR & B0R085 & 4 & Habp4 & Habp4 & 557.83 & 267 & 273 \\
\hline 10 & 2 & KVVNPLFE[K]RPK & A0A2R8Q9K6 & 9 & FGQLLGDESDPFDILYAAGTE[K]K & B0R085 & 22 & Rpl7a & Habp4 & 375.33 & 34 & 42 \\
\hline 8 & 2 & LFCVGFT[K]K & Q6PBY1 & 8 & 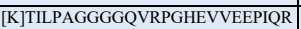 & B0R085 & 1 & Rps3a & Habp4 & 311.29 & 144 & 70 \\
\hline 6 & 2 & DYG[K]ESQAK & Q6PC90 & 4 & ALQEQSRP[K]VELNIR & B0R085 & 9 & Rps12 & Habp4 & 356.42 & 116 & 246 \\
\hline 6 & 2 & LMELHGEGGSTSAAA[K]PAEGDTGAK & Q6PBY1 & 16 & [K]TILPAGGGGQVRPGHEVVEEPIQR & B0R085 & 1 & Rps3a & Habp4 & 251.05 & 243 & 70 \\
\hline 5 & 2 & DVIEEYF[K]SK & Q6PC90 & 8 & ALQEQSRP[K]VELNIR & B0R085 & 9 & Rps12 & Habp4 & 436.68 & 129 & 246 \\
\hline 4 & 1 & KKEEP[K]K & B0R085 & 6 & TSTTT[K]SVK & B0R085 & 6 & Habp4 & Habp4 & 326.43 & 52 & 59 \\
\hline 3 & 1 & $\overline{\mathrm{AVVIH}}[\mathrm{K}] \mathrm{SK}$ & B0R085 & 6 & [K]TESSVPSK & B0R085 & 1 & Habp4 & Habp4 & 339.89 & 267 & 253 \\
\hline 3 & 1 & [K]TESSVPSK & B0R085 & 1 & ALQEQSRP[K]VELNIR & B0R085 & 9 & Habp4 & Habp4 & 456.48 & 253 & 246 \\
\hline 3 & 2 & AVVIH[K]SK & B0R085 & 6 & [G]HQQLYWSHPR & Q6Q419 & 1 & Habp4 & Rps29 & 413.44 & 267 & 2 \\
\hline 3 & 2 & IVV[K]VLK & Q7ZWJ7 & 4 & FGQLLGDESDPFDILYAAGTE[K]K & B0R085 & 22 & Rpl34 & Habp4 & 315.68 & 105 & 42 \\
\hline 3 & 2 & [K]VVNPLFEK & \begin{tabular}{|l|} 
A0A2R8Q9K6 \\
\end{tabular} & 1 & FGQLLGDESDPFDILYAAGTE[K]K & B0R085 & 22 & Rpl7a & Habp4 & 263.18 & 26 & 42 \\
\hline 3 & 2 & EVQTNDL[K]EVVNK & Q6PBY1 & 8 & 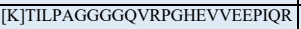 & B0R085 & 1 & Rps3a & Habp4 & 376.9 & 182 & 70 \\
\hline 3 & 2 & AMELL[K]VSK & Q6Q415 & 6 & [K]TILPAGGGGQVRPGHEVVEEPIQR & B0R085 & 1 & Rpl36 & Habp4 & 270.36 & 62 & 70 \\
\hline 2 & 1 & KKEEP[K]K & B0R085 & 6 & TSTTTKSV[K]K & B0R085 & 9 & Habp4 & Habp4 & 349.79 & 52 & 62 \\
\hline 2 & 2 & K[K]EEPKK & B0R085 & 2 & AQAQTQ[K]SK & $\overline{\text { Q7ZWJ7 }}$ & 7 & Habp4 & Rpl34 & 265.64 & 48 & 115 \\
\hline 2 & 2 & VL[K]AQAQTQK & Q7ZWJ7 & 3 & FGQLLGDESDPFDILYAAGTE[K]K & B0R085 & 22 & Rpl34 & Habp4 & 390.92 & 108 & 42 \\
\hline 1 & 2 & AVVIH[K]SK & B0R085 & 6 & SAAQPGAD[K]K & Q7T1J9 & 9 & Habp4 & Rps10 & 354.31 & 267 & 139 \\
\hline 1 & 2 & [K]KKEEPK & B0R085 & 1 & VVNPLFE[K]RPK & \begin{tabular}{|l|l} 
A0A2R8Q9K6 \\
\end{tabular} & 8 & Habp4 & Rpl7a & 244.17 & 46 & 34 \\
\hline 1 & 2 & AVVIH[K]SK & B0R085 & 6 & [K]AEAGAGAATEFQFR & Q7T1J9 & 1 & Habp4 & Rps10 & 247.31 & 267 & 140 \\
\hline 1 & 3 & $\overline{\mathrm{APP}}[\mathrm{K}] \mathrm{PM}$ & \begin{tabular}{|l|} 
F1QHJ1;Q6P6E2 \\
\end{tabular} & 4 & $\overline{[K] T I L P A G G G G Q V R P G H E V V E E P I Q R ~}$ & B0R085 & 1 & Rps26 & Habp4 & 228.16 & $113 ; 113$ & 70 \\
\hline 1 & 2 & VWNLANC[K]LK & 042248 & 8 & SPQ[K]FDSAPNPDDPEDFPALA & B0R085 & 4 & Gnb211 & Habp4 & 497.46 & 183 & 330 \\
\hline 2 & 1 & HQSPIEVPD[K]KDDK & F1QHJ4 & 10 & DSTEYETVIPP[K]LPVVVSGVVTK & F1QHJ4 & 12 & Dap & Dap & 395.01 & 39 & 55 \\
\hline 2 & 2 & VTGGAAS[K]LSK & A0A2R8RS24 & 8 & DSTEYETVIPP[K]LPVVVSGVVTK & F1QHJ4 & 12 & Rpl35 & Dap & 285.52 & 42 & 55 \\
\hline 4 & 2 & LN[K]AVWAK & Q24JV3 & 3 & AGHLPAV[K]AGGMR & F1QHJ4 & 8 & Rpl31 & Dap & 351.4 & 68 & 20 \\
\hline 2 & 1 & KTD[K]PR & F1QEQ3 & 4 & SADENANVE[K]ETR & F1QEQ3 & 10 & Daplb & Daplb & 192.48 & 29 & 22 \\
\hline
\end{tabular}

Habp4 crosslinks are highlighted in blue, Dap/Dap1b crosslinks are depicted in green. 
bioRxiv preprint doi: https://doi.org/10.1101/2021.11.03.467131; this version posted November 3, 2021. The copyright holder for this

preprint (which was not certified by peer review) is the author/funder, who has granted bioRxiv a license to display the preprint in perpetuity. It is made available under aCC-BY-NC 4.0 International license.

Extended Data Table 4. Crosslinks identified for Habp4 in ribosome samples from Xenopus eggs.

\begin{tabular}{|c|c|c|c|c|c|c|c|c|c|c|c|c|}
\hline \#CSMs & \#Proteins & Sequence $\mathrm{A}$ & Accession A & $\begin{array}{c}\text { Position } \\
\text { A }\end{array}$ & Sequence B & Accession B & $\begin{array}{c}\text { Position } \\
\text { B }\end{array}$ & $\begin{array}{c}\text { Protein } \\
\text { A }\end{array}$ & $\begin{array}{c}\text { Protein } \\
\text { B }\end{array}$ & $\begin{array}{l}\text { Best } \\
\text { CSM } \\
\text { Score }\end{array}$ & $\begin{array}{c}\text { In } \\
\text { protein A }\end{array}$ & $\begin{array}{c}\text { In } \\
\text { protein B }\end{array}$ \\
\hline 1 & 2 & VTQQTGQ[K]R & A0A1L8HPG3 & 8 & LMELHGEGGGSGKPAADETGA[K]VER & A0A1L8HUF5 & 22 & Habp4 & Rps3a & 385.36 & 99 & 249 \\
\hline 1 & 2 & \begin{tabular}{|l} 
VTQNENVDSQV[K]VDR \\
\end{tabular} & \begin{tabular}{|l|l|} 
A0A1L8HPG3 \\
\end{tabular} & 12 & LMELHGEGGGTGKPAGDETGA[K]VER & A0A1L8HLR2 & 22 & Habp4 & Rps3a & 424.77 & 119 & 249 \\
\hline 1 & 2 & \begin{tabular}{|l} 
NTE[K]VTQNENVDDSQVK \\
\end{tabular} & A0A1L8HPG3 & 4 & LMELHGEGGGTGKPAGDETGA[K]VER & A0A1L8HLR2 & 22 & Habp4 & Rps3a & 429.73 & 107 & 249 \\
\hline 1 & 3 & AVVIH[K]SK & $\begin{array}{l}\text { A0A1L8HPG3; } \\
\text { A0A1L8HYF8 }\end{array}$ & 6 & SAVPSGAD $[\mathrm{K}] \mathrm{K}$ & B7ZPG6 & 9 & Habp4 & Rps10 & 299.5 & $297 ; 323$ & 138 \\
\hline 1 & 3 & [K]AVVVGGSTDVK & A0A1L8HPG3 & 1 & EVQTNDL[K]EVVNK & A0A1L8HLR2;A0A1L8HUF5 & 8 & Habp4 & Rps3a & 569.03 & 80 & $182 ; 182$ \\
\hline 1 & 2 & $\mathrm{~S}[\mathrm{~K}] \mathrm{TELNLR}$ & \begin{tabular}{|l|} 
A0A1L8HPG3 \\
\end{tabular} & 2 & VVGCSCVVV[K]DYGK & $\mathrm{A} 0 \mathrm{~A} 310 \mathrm{U} 0 \mathrm{~K} 2$ & 10 & Habp4 & Rps12 & 280.87 & 276 & 78 \\
\hline 1 & 3 & $\begin{array}{l}\mathrm{VTQQTGQ}[\mathrm{K}] \mathrm{R} \\
\end{array}$ & A0A1L8HPG3 & 8 & LITEDVQG[K]NCLTNFHGMDLTR & A0A1L8HLR2;A0A1L8HUF5 & 9 & Habp4" & Rps3a & 314.68 & 99 & $94 ; 94$ \\
\hline 1 & 3 & \begin{tabular}{|l|l|} 
VTQNENVDSQV[K]VDR \\
\end{tabular} & A0A1L8HPG3 & 12 & LITEDVQG[K]NCLTNFHGMDLTR & A0A1L8HLR2;A0A1L8HUF5 & 9 & Habp4 & Rps3a & 819.16 & 119 & $94 ; 94$ \\
\hline 1 & 2 & SAEYSIE[K]PMEIMDQDK & A0A1L8HPG3 & 8 & V[K]ADRDESSPYAAMLAAQDVAQR & Q6PI76 & 2 & Habp4 & $\begin{array}{c}\text { Rps14- } \\
\text { prov }\end{array}$ & 674.31 & 147 & 63 \\
\hline 1 & 2 & \begin{tabular}{|l|} 
VTQNENVDSQV[K]VDR \\
\end{tabular} & \begin{tabular}{|l|} 
A0A1L8HPG3 \\
\end{tabular} & 12 & DISEASVFDSYALP[K]LYVK & Q6AZL7 & 15 & Habp4 & Rps26 & 752.94 & 119 & 66 \\
\hline 1 & 2 & DVHMP[K]HPELVDK & A0A1L8H6M8 & 6 & KPETSVPS[K]AVVIHK & A0A1L8HPG3 & 9 & Rps10 & Habp4 & 333.09 & 350 & 291 \\
\hline 1 & 2 & AQAQSQ[K]AK & A0A1L8HUR4 & 7 & K[K]EEGAAKK & A0A1L8HPG3 & 2 & \begin{tabular}{|l|l|} 
Rpl134 \\
\end{tabular} & Habp4 & 298.13 & 151 & 57 \\
\hline 1 & 2 & A[K]ELATK & \begin{tabular}{|l|} 
A0A1L8F1B99 \\
\end{tabular} & 2 & [K]AVVVGGSTDVK & A0A1L8HPG3 & 1 & Rpl7a & Habp4 & 270.61 & 286 & 80 \\
\hline 1 & 3 & EVQTNDL[K]EVVNK & $\begin{array}{l}\text { A0A1L8HLR2; } \\
\text { A0A1L8HUF5 } \\
\end{array}$ & 8 & VTQNENVDSQV[K]VDR & A0A1L8HPG3 & 12 & Rps3a & Habp4 & 537.7 & $182 ; 182$ & 119 \\
\hline 1 & 3 & LFCVGFT[K]K & $\begin{array}{l}\text { A0A1L8HLR2; } \\
\text { A0A1L8HUF5 } \\
\end{array}$ & 8 & VTQNENVDSQV[K]VDR & A0A1L8HPG3 & 12 & Rps3a & Habp4 & 424.76 & $144 ; 144$ & 119 \\
\hline 1 & 2 & [K]AAVVGGNTDVK & A0A1L8HYF8 & 1 & LMELHGEGGGTGKPAGDETGA[K]VER & $\begin{array}{l}\text { A0A1L8HLR2 } \\
\end{array}$ & 22 & Habp4 & Rps3a & 418.66 & 80 & 249 \\
\hline 1 & 2 & [K]AAVVGGNTDVK & A0A1L8HYF8 & 1 & LMELHGEGGGTG[K]PAGDETGAK & A0A1L8HLR2 & 13 & Habp4 & Rps3a & 604.07 & 80 & 240 \\
\hline 1 & 2 & \begin{tabular}{|l} 
APQNENVGSQV[K]VDR \\
\end{tabular} & A0A1L8HYF8 & 12 & DNYVPEVSALDQEIIEVDPDT[K]EMLK & A0A1L8H046 & 22 & Habp4 & Rps17 & 441.32 & 145 & 103 \\
\hline 1 & 2 & AQAQSQ[K]AK & \begin{tabular}{|l|} 
A0A1L8HUR4 \\
\end{tabular} & 7 & K[K]EDAAAKK & A0A1L8HYF8 & 2 & Rpl34 & Habp4 & 451.09 & 151 & 57 \\
\hline 1 & 3 & EVQTNDL[K]EVVNK & $\begin{array}{l}\text { A0A1L8HLR2; } \\
\text { A0A1L8HUF5 }\end{array}$ & 8 & APQNENVGSQV[K]VDR & A0A1L8HYF8 & 12 & Rps3a & Habp4 & 542.26 & $182 ; 182$ & 145 \\
\hline 1 & 3 & LFCVGFT[K]K & $\begin{array}{l}\text { A0A1L8HLR2; } \\
\text { A0A1L8HUF5 }\end{array}$ & 8 & APQNENVGSQV[K]VDR & A0A1L8HYF8 & 12 & Rps3a & Habp4 & 443.3 & $144 ; 144$ & 145 \\
\hline 1 & 1 & NANQ[K]SGK & A0A1L8HPG3 & 5 & [K]AVVVGGSTDVK & A0A1L8HPG3 & 1 & Habp4 & Habp4 & 298.57 & 69 & 80 \\
\hline 1 & 1 & KPEAAVPT[K]AVVIHK & A0A1L8HYF8 & 9 & F[K]NNLSENEEDYHYCFR & A0A1L8HYF8 & 2 & Habp4 & Habp4 & 365.16 & 317 & 327 \\
\hline 1 & 1 & [K]AAVVGGNTDVK & A0A1L8HYF8 & 1 & MTPQTESG[K]VSQK & A0A1L8HYF8 & 9 & Habp4 & Habp4 & 637.08 & 80 & 100 \\
\hline 1 & 1 & VTQPGQ[K]HAPK & A0AIL8HYF8 & 7 & [K]TFVVGGNTDVK & A0A1L8HYF8 & 1 & \begin{tabular}{|l|} 
Habp 4 \\
\end{tabular} & Habp4 & 311.71 & 125 & 107 \\
\hline 1 & 1 & [K]TFVVGGNTDVK & A0A1L8HYF8 & 1 & MTPQTESG[K]VSQK & A0A1L8HYF8 & 9 & Habp4 & Habp4 & 358.27 & 107 & 100 \\
\hline 2 & 3 & VTQQTGQ[K]R & \begin{tabular}{|l|} 
A0A1L8HPG3 \\
\end{tabular} & 8 & EVQTNDL[K]EVVNK & A0A1L8HLR2;A0A1L8HUF5 & 8 & Habp4 & Rps3a & 402.91 & 99 & $182 ; 182$ \\
\hline 2 & 2 & K[K]EEGAAK & A0A1L8HPG3 & 2 & $\mathrm{VL}[\mathrm{K}] \mathrm{AQAQQSQK}$ & A0A1L8HUR4 & 3 & Habp4 & Rpl34 & 398.43 & 57 & 144 \\
\hline 2 & 2 & K[K]EEGAAK & A0A1L8HPG3 & 2 & VAPAPSVV[K]K & A0A1L8F1B9 & 9 & Habp4 & Rpl7a & 268.6 & 57 & 47 \\
\hline 2 & 2 & RAP[K]NTEK & \begin{tabular}{|l|} 
A0A1L8HPG3 \\
\end{tabular} & 4 & LMELHGEGGGTG[K]PAGDETGAK & A0A1L8HLR2 & 13 & Habp4 & Rps3a & 271.82 & 103 & 240 \\
\hline 2 & 2 & NTE[K]VTQNENVDSQVK & A0A1L8HPG3 & 4 & LMELHGEGGGTG[K]PAGDETGAK & A0A1L8HLR2 & 13 & Habp4 & Rps3a & 766.1 & 107 & 240 \\
\hline 2 & 4 & AVVIH[K]SK & $\begin{array}{l}\text { A0A1L8HPG3; } \\
\text { A0A1L8HYF8 } \\
\end{array}$ & 6 & [K]AEAGAGAATEFQFR & $\begin{array}{c}\text { B7ZPG6; } \\
\text { A0A1L8H6M8 }\end{array}$ & 1 & Habp4 & Rps10 & 347.49 & $297 ; 323$ & $139 ; 458$ \\
\hline 2 & 2 & VTQQTGQ[K]R & A0A1L8HPG3 & 8 & DIE[K]ACQSIYPLHDVYVR & A0A1L8HLR2 2 & 4 & Habp4 & Rps3a & 276 & 99 & 199 \\
\hline 2 & 2 & SAEYSIE[K]PMEIMDQDK & A0A1L8HPG3 & 8 & DISEASVFDSYALP[K]LYVK & Q6AZL7 & 15 & Habp4 & Rps26 & 658.25 & 147 & 66 \\
\hline 2 & 3 & LFCVGFT[K]K & $\begin{array}{l}\text { A0A1L8HLR2; } \\
\text { A0A1L8HUF5 } \\
\end{array}$ & 8 & NTE[K]VTQNENVDSQVK & A0A1L8HPG3 & 4 & Rps3a & Habp4 & 541.44 & $144 ; 144$ & 107 \\
\hline 2 & 4 & $\mathrm{~A}[\mathrm{~K}]$ ELATK & \begin{tabular}{|l|} 
A0A1L8F1B99 \\
\end{tabular} & 2 & ESQ[K]DRK & A0A1L8HPG3;A0A1L8HYF8 & 4 & Rpl7a & Habp4 & 288.23 & 286 & $66 ; 77 ; 77$ \\
\hline 2 & 3 & NLG[K]TLVTR & $\begin{array}{l}\text { A0A1L8HLR2; } \\
\text { A0A1L8HUF5 } \\
\end{array}$ & 4 & VTQNENVDSQV[K]VDR & A0A1L8HPG3 & 12 & Rps3a & Habp4 & 305.26 & $46 ; 46$ & 119 \\
\hline 2 & 3 & DWYDV[K]APAMFNIR & $\begin{array}{l}\text { A0A1L8HLR2; } \\
\text { A0A1L8HUF5 }\end{array}$ & 6 & NTE[K]VTQNENVDSQVK & A0A1L8HPG3 & 4 & Rps3a & Habp4 & 676.92 & $34 ; 34$ & 107 \\
\hline 2 & 3 & DWYDV[K]APAMFNIR & $\begin{array}{l}\text { A0A1L8HLR2; } \\
\text { A0A1L8HUF5 }\end{array}$ & 6 & VTQNENVDSQV[K]VDR & A0A1L8HPG3 & 12 & Rps3a & Habp4 & 606.91 & $34 ; 34$ & 119 \\
\hline 2 & 3 & K[K]KEDAAAK & A0A1L8HYF8 & 2 & VVNPLFE[K]RPK & \begin{tabular}{|l|} 
A0A1L8F671;A0A1L8F1B9 \\
\end{tabular} & 8 & Habp4 & Rpl7a & 305.57 & 56 & $34 ; 61$ \\
\hline 2 & 2 & SNEYSIE[K]PMEILDQDK & A0A1L8HYF8 & 8 & V[K]ADRDESSPYAAMLAAQDVAQR & Q6PI76 & 2 & Habp4 & $\begin{array}{c}\text { Rps14- } \\
\text { prov }\end{array}$ & 820.86 & 173 & 63 \\
\hline 2 & 3 & VA[K]SNR & $\begin{array}{c}\text { Q7SYU3; } \\
\text { A0A1L8F8E2 }\end{array}$ & 3 & APQNENVGSQV[K]VDR & A0A1L8HYF8 & 12 & Rps5 & Habp4 & 294.66 & $200 ; 200$ & 145 \\
\hline 2 & 1 & KPETSVPS[K]A & A0A1L8HPG3 & 9 & $\mathrm{Y}[\mathrm{K}$ & $\mathrm{A} 0$ & 2 & Iabp4 & Habp4 & 0.87 & 291 & 301 \\
\hline 2 & 1 & VTQPGQ[K]HAPK & A0A1L8HYF8 & 7 & NME[K]APQNENVGSQVK & A0A1L8HYF8 & 4 & Habp4 & Habp4 & 388.06 & 125 & 133 \\
\hline 3 & 2 & [K]AVVVGGSTDVK & A0A1L8HPG3 & 1 & LMELHGEGGGTGKPAGDETGA[K]VER & A0A1L8HLR2 & 22 & Habp4 & Rps3a & 400.54 & 80 & 249 \\
\hline 3 & 2 & VTQQTGQ[K]R & A0A1L8HPG3 & 8 & LMELHGEGGGTG[K]PAGDETGAK & A0A1L8HLR2 & 13 & Habp4 & Rps3a & 394.43 & 99 & 240 \\
\hline 3 & 3 & QNSLL[K]R & A0A1L8HNA1 & 6 & AVVIH[K]SK & A0A1L8HPG3;A0A1L8HYF8 & 6 & Rps15 & Habp4 & 318.44 & 68 & $297 ; 323$ \\
\hline 3 & 3 & APQNENVGSQV[K]VDR & A0A1L8HYF8 & 12 & DWYDV[K]APAMFNIR & \begin{tabular}{|l|} 
A0A1L8HLR2;A0A1L8HUF5 \\
\end{tabular} & 6 & Habp4 & Rps3a & 534.34 & 145 & $34 ; 34$ \\
\hline 3 & 3 & KKEDAAA $[\mathrm{K}] \mathrm{K}$ & A0A1L8HYF8 & 8 & VVNPLFE[K]RPK & \begin{tabular}{|l|} 
A0A1L8F671;A0A1L8F1B9 \\
\end{tabular} & 8 & Habp4 & Rpl7a & 264.49 & 63 & $34 ; 61$ \\
\hline 3 & 3 & K[K]EDAAAKK & A0A1L8HYF8 & 2 & VVNPLFE[K]RPK & \begin{tabular}{|l|} 
A0A1L8F671;A0A1L8F1B9 \\
\end{tabular} & 8 & Habp4 & Rpl7a & 277.77 & 57 & $34 ; 61$ \\
\hline 3 & 3 & AIRPED[K]R & A0A1L8HYF8 & 7 & Y[K]LLGGLAVR & A0A1L8HJY0;Q7ZYT3 & 2 & Habp4 & Rps3 3 & 286.15 & 231 & $108 ; 108$ \\
\hline 3 & 2 & VT[K]VLGR & Q66KP7 & 3 & APQNENVGSQV[K]VDR & A0A1L8HYF8 & 12 & Rps28 & Habp4 & 298.45 & 16 & 145 \\
\hline 3 & 1 & VTQQTGQ[K]R & A0A1L8HPG3 & 8 & $\begin{array}{l}\text { VTQNENVDSQV[K]VDR } \\
\end{array}$ & A0A1L8HPG3 & 12 & \begin{tabular}{|l|l|} 
Habp 4 \\
\end{tabular} & Habp4 & 430.73 & 99 & 119 \\
\hline 3 & 1 & RAP[K]NTEK & A0A1L8HPG3 & 4 & AVVVGGSTDV[K]VTQQTGQK & A0A1L8HPG3 & 11 & Habp4 & Habp4 & 319.01 & 103 & 91 \\
\hline 4 & 3 & AVVIH[K]SK & $\begin{array}{r}\text { A0A1L8HPG3; } \\
\text { A0A1L8HYF8 }\end{array}$ & 6 & [G]HQQLYWSHPR & A0A1L8F058 & 1 & Habp4 & Rps29 & 353.32 & $297 ; 323$ & 2 \\
\hline 4 & 3 & TQNVLGE[K]GR & $\begin{array}{l}\text { A0AIL8HJY0; } \\
\text { O7ZYT3 }\end{array}$ & 8 & VPEE[K]NEGFSQEMSLDEWR & A0A1L8HYF8 & 5 & Rps3 & Habp4 & 475.95 & $62 ; 62$ & 279 \\
\hline 4 & 2 & CCLTYCFN[K]PEDK & Q6GMC1 & 9 & KPEAAVPT[K]AVVIHK & A0A1L8HYF8 & 9 & Rps27a & Habp4 & 550.91 & 152 & 317 \\
\hline 5 & 3 & VTQQTGQ[K]R & A0A1L8HPG3 & 8 & DWYDV[K]APAMFNIR & A0A1L8HLR2;A0A1L8HUF5 & 6 & Habp4 & Rps3a & 414 & 99 & $34 ; 34$ \\
\hline 5 & 2 & DVHMP[K]HPELADK & $\mathrm{B} 7 \mathrm{Z}$ & 6 & Y[K]NNISENEEESQYCFR & A0A1L8HPG3 & 2 & Rps10 & Habp4 & 558.94 & 31 & 301 \\
\hline 5 & 2 & VT[K]VLGR & Q66KP7 & 3 & SAEYSIE[K]PMEIMDQDK & A0A1L8HPG3 & 8 & Rps 28 & $\begin{array}{ll}\text { Habp4 } \\
\end{array}$ & 312.98 & 16 & 147 \\
\hline 5 & 2 & CCLTYCFN[K]PEDK & Q6GMC1 & 9 & KPETSVPS[K]AVVIHK & A0A1L8HPG3 & 9 & Rps27a & Habp4 & 627.11 & 152 & 291 \\
\hline 5 & 2 & VT[K]VLGR & Q66KP7 & 3 & SNEYSIE[K]PMEILDQDK & A0A1L8HYF8 & 8 & Rps28 & Habp4 & 270.61 & 16 & 173 \\
\hline 5 & 2 & DVHMP[K]HPELADK & B7ZPG6 & 6 & F[K]NNLSENEEDYHYCFR & A0A1L8HYF8 & 2 & \begin{tabular}{|l|l|} 
Rps 10 \\
\end{tabular} & Habp4 & 564.39 & 31 & 327 \\
\hline 5 & 1 & VTQQTGQ[K]R & A0A1L8HPG3 & 8 & NTE[K]VTQNENVDSQVK & A0A1L8HPG3 & 4 & Habp4 & Habp4 & 401.36 & 99 & 107 \\
\hline 6 & 2 & VTQQTC & & 8 & LMELHGEGGGTGKPAGDETGA[K]VER & HLR2 & 22 & Habp4 & Rps3a & 403.65 & 99 & 249 \\
\hline 6 & 2 & S[K]TELNLR & $\begin{array}{ll}\text { A0A1L8HPG3 } \\
\end{array}$ & 2 & DYG[K]ESQAK & $\mathrm{A} 0 \mathrm{~A} 310 \mathrm{U} 0 \mathrm{~K} 2$ & 4 & Habp4 & Rpsi2 & 303.71 & 276 & 82 \\
\hline 6 & 2 & $\mathrm{~S}[\mathrm{~K}] \mathrm{]}$ & & 2 & & & 8 & Habp4 & Rps12 & 302.12 & 276 & 95 \\
\hline 6 & 2 & $\mathrm{~K}[\mathrm{~K}] \mathrm{E}$ & & 2 & $\mathrm{AQAC}$ & BHUR4 4 & 7 & Habp4 & Rpl34 & 554.03 & 57 & 151 \\
\hline 6 & 2 & DVHMP[K]HPELVDK & A0A1L8H6M8 & 6 & F[K]NNLSENEEDYHYCFR & A0A1L8HYF8 & 2 & Rps10 & Habp4 & 435.86 & 350 & 327 \\
\hline 6 & 3 & ESQ[K]DRK & $\begin{array}{l}\text { A0A1L8HPG3; } \\
\text { A0A1L8HYF8 }\end{array}$ & 4 & VTQQTGQ[K]R & A0A1L8HPG3 & 8 & Habp4 & Habp4 & 265.8 & \begin{tabular}{|l|}
$66 ; 77 ; 77$ \\
\end{tabular} & 99 \\
\hline 7 & 2 & K[K]EEGAAK & A0A1L8HPG3 & 2 & AQAQSQ[K]AK & A0A1L8HUR4 & 7 & Habp4 & Rpl34 & 416.25 & 57 & 151 \\
\hline 7 & 1 & VTQQTGQ[K]R & \begin{tabular}{|l|} 
A0A1L8HPG3 \\
\end{tabular} & 8 & [K]AVVVGGSTDVK & L8HPG3 & 1 & Habp4 & $\begin{array}{ll}\text { Habp4 } \\
\end{array}$ & 460.72 & 99 & 80 \\
\hline 7 & 1 & RAP[K]NTEK & A0A1L8HPG3 & 4 & VTQNENVDSQV[K]VDR & A0A1L8HPG3 & 12 & Habp4 & Habp4 & 392.28 & 103 & 119 \\
\hline 7 & 1 & S[K]TELNLR & \begin{tabular}{|l|} 
A0A1L8HPG3 \\
\end{tabular} & 2 & [K]PETSVPSK & A0A1L8HPG3 & 1 & \begin{tabular}{|l|} 
Habp4 \\
\end{tabular} & $\begin{array}{ll}\text { Habp4 } \\
\end{array}$ & 271.28 & 276 & 283 \\
\hline 8 & 3 & LFCVGFT[K]K & $\begin{array}{r}\text { A0A1L8HLR2; } \\
\text { A0A1L8HUF5 }\end{array}$ & 8 & VTQQTGQ[K]R & A0A1L8HPG3 & 8 & Rps3a & Habp4 & 478.54 & $144 ; 144$ & 99 \\
\hline 8 & 2 & AVVIH[K]SK & $\begin{array}{l}\text { A0A1L8HPG3; } \\
\text { A0A1L8HYF8 }\end{array}$ & 6 & Y[K]NNISENEEESQYCFR & A0A1L8HPG3 & 2 & Habp4 & Habp4 & 478.53 & $297 ; 323$ & 301 \\
\hline 9 & 1 & {$[\mathrm{~K}] \mathrm{SANQK}$} & A0A1 & 1 & K[K]EDAAAK & $\begin{array}{l}\text { A0A1L8HYF8 } \\
\end{array}$ & 2 & Habp4 & Habp4 & 324.03 & 64 & 57 \\
\hline 10 & 1 & 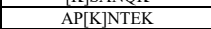 & $\mathrm{A} 0 \mathrm{~A}$ & 3 & $\mathbf{G Q}[\mathrm{K}] \mathrm{R}$ & $\mathrm{HPG} 3$ & 8 & \begin{tabular}{|l|} 
Habp4 \\
\end{tabular} & Habp4 & 285.76 & 103 & 99 \\
\hline 12 & 3 & K[K]EEGAAK & A0A1L8HPG3 & 2 & KVVNPLFE[K]RPK & \begin{tabular}{|l|} 
A0A1L8F671;A0A1L8F1B9 \\
\end{tabular} & 9 & Habp4 & Rpl7a & 345.57 & 57 & $34 ; 61$ \\
\hline 13 & 2 & KDVHMP[K]HPELVDK & $\begin{array}{l}\text { A0A1L8H6M8 } \\
\end{array}$ & 7 & Y[K]NNISENEEESQYCFR & A0A1L8HPG3 & 2 & \begin{tabular}{|l|} 
Rps 10 \\
\end{tabular} & Habp4 & 715.72 & 350 & 301 \\
\hline 14 & 2 & AVVIH[K]SK & $\begin{array}{l}\text { A0A1L8HPG3; } \\
\text { A0A1L8HYF8 }\end{array}$ & 6 & F[K]NNLSENEEDYHYCFR & A0A1L8HYF8 & 2 & Habp4 & Habp4 & 477.14 & $297 ; 323$ & 327 \\
\hline
\end{tabular}


bioRxiv preprint doi: https://doi.org/10.1101/2021.11.03.467131; this version posted November $3,2021$. The copyright holder for this

preprint (which was not certified by peer review) is the author/funder, who has granted bioRxiv a license to display the preprint in perpetuity. It is made available under aCC-BY-NC 4.0 International license.

Extended Data Table 5. Crosslinks identified for Dapl1 in ribosome samples from Xenopus eggs.

\begin{tabular}{|c|c|c|c|c|c|c|c|c|c|c|c|c|}
\hline \#CSMs & \#Proteins & Sequence $\mathbf{A}$ & Accession A & $\begin{array}{l}\text { Position } \\
\text { A }\end{array}$ & Sequence B & Accession B & $\begin{array}{c}\text { Position } \\
\text { B }\end{array}$ & Protein A & Protein B & \begin{tabular}{|c|} 
Best \\
CSM \\
Score \\
\end{tabular} & $\begin{array}{c}\text { In protein } \\
\text { A }\end{array}$ & $\begin{array}{c}\text { In protein } \\
\text { B }\end{array}$ \\
\hline 1 & 2 & EL[K]VQSSPQALK & A0A1L8EP22 & 3 & [K]RNEDEDSPNK & A0A1L8H8P5 & 1 & DapL1.S & Rpl31 & 430.89 & 6 & 113 \\
\hline 18 & 4 & LN[K]AVWAK & $\begin{array}{l}\text { A0A1L8EJF0; } \\
\text { A0A1L8H8P5 }\end{array}$ & 3 & AGHLPAV[K]AGGMR & $\begin{array}{l}\text { A0A1L8EP22; } \\
\text { A0A1L8EW14 }\end{array}$ & 8 & Rpl31 & DapL1.S & 486.09 & $55 ; 92$ & $23 ; 23$ \\
\hline 6 & 3 & VTGGAAS[K]LSK & A0A1L8F6D5 & 8 & TLQE[K]PSSVLNMTK & $\begin{array}{l}\text { A0A1L8EP22; } \\
\text { A0A1L8EW14 }\end{array}$ & 5 & Rpl35 & DapL1.S & 527.01 & 57 & $52 ; 52$ \\
\hline 2 & 2 & [K]RNEDEDSPNK & A0A1L8H8P5 & 1 & KQGNDENSAPE[K]NAK & A0A1L8EP22 & 12 & Rpl31 & DapL1.S & 354.91 & 113 & 43 \\
\hline 5 & 2 & [K]RNEDEDSPNK & A0A1L8H8P5 & 1 & $\begin{array}{l}\text { KQGNEENSAPE[K]NAK } \\
\end{array}$ & A0A1L8EW14 & 12 & Rpl31 & \begin{tabular}{|l|} 
DapL1.L \\
\end{tabular} & 412.98 & 113 & 43 \\
\hline 2 & 2 & [K]RNEDEDSPNK & A0A1L8H8P5 & 1 & EQ[K]MQSSPQALK & A0A1L8EW14 & 3 & Rpl31 & DapL1.L & 495.57 & 113 & 6 \\
\hline
\end{tabular}

Extended Data Table 6. Primers used in this study.

\begin{tabular}{|l|l|}
\hline Oligo name & Sequence (5' to 3') \\
\hline dap_gRNA1 & GTCTCGGTTTTCTCTTTGGG \\
\hline dap_gRNA2 & CATGGGCATTACCAGCAGGAAGG \\
\hline dap1b_gRNA1 & GTCATCCACCTGCAGGTTTGGG \\
\hline dap1b_gRNA2 & GACGAAATCCCCAAACCTGCAGG \\
\hline habp4_gRNA1 & ATATAGGATGTCGAAAGGGTCGG \\
\hline habp4_gRNA2 & ACCTTGTCCGCCCCCACCTGCGG \\
\hline common_tracer_oligo & $\begin{array}{l}\text { AAAAGCACCGACTCGGTGCCACTTTTTCAAGTTGATAACG } \\
\text { GACTAGCCTTATTTTAACTTGCTATTTCTAGCTCTAAAAC }\end{array}$ \\
\hline dap_gt_F & AGTCGACGGTAGTCTGCGTTAT \\
\hline dap_gt_R & TCAAAAAGGTGCCATTTAGTGT \\
\hline dap1b_gt_F & GTGTTTTTGTGAGAAGCGTTTG \\
\hline dap1b_gt_R & CACCATTTGGTCAATAGACGTG \\
\hline habp4_gt_F & GATGAGGGATATGGATGCACCGTGG \\
\hline habp4_gt_R & TCAGTCCCTGCAGCATATAGG \\
\hline
\end{tabular}

